\title{
AROMATIC HYDROXYLATION AS
}

AN ANALYTICAL REACTION

by

KENNETH STEPHEN ATBERT

A thesis submitted in partial fulfillment of the requirements for the degree of

DOCTOR OF PHILOSOPHY

(Pharmacy)

at the

UNIVERSITY OF WISCONSIN

1972 
I wish to express my sincere gratitude to Professor Kenneth A. Connors for his guidance and suggestions during the course of this investigation. I also appreciate his patience and interest in the development of my scientific career.

- The technical assistance of Mr. Ronald E. Behling is gratefully acknowledged.

Financial support was provided by the Research Committee of the University of Wisconsin. 
"If someone tells me that in making these conclusions I have gone beyond the facts, I reply: 'it is true that I have freely put myself among ideas which cannot be rigorously proved. That is my way of looking at things.'" - IOUIS PASTEUR

With this thought, I dedicate this thesis to

my mother, my wife Evie and my daughter Heidi Ellyn. 
TABLE OF CONTENTS

Aromatic Hydroxylation as an Analytical Reaction

Page

I. INTRODUCTION $\ldots \ldots \ldots \ldots \ldots \ldots \ldots \ldots \ldots \ldots \ldots \ldots \ldots \ldots \ldots \ldots \ldots \ldots$

A. Aromatic Hydroxylation Systems ........... 2

- B. Methods for Phenol Determination ......... 12

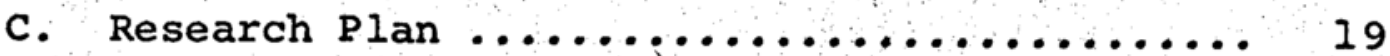

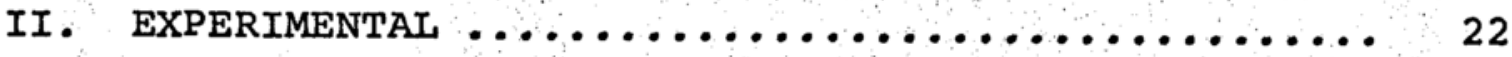

A. Materials ....................... 22

B. Apparatus ........................ 26

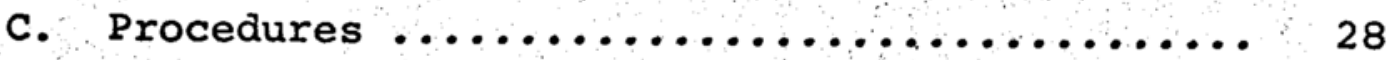

1. Hydroxylation Time course studies. .... 28

2. Proposed Analytical Method. .......... 29

3. Studies on the Analytical Finish. .... 30

(a) The 4-Aminoantipyrine Method. .... 30

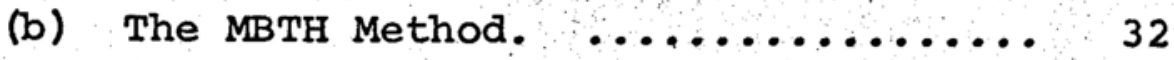

4. Attempted TLC Detection Studies. ..... 32

A. Hydroxylation of Anisole with the Hamilton System

1. Dependence of Time Course Appearance on Peroxide/Substrate Ratio.

2. Dependence of Yield on Catechol and Fe (III) Concentrations.

3. Dependence of Yield on $\mathrm{pH}$.

4. "Aging" of the Hamilton Reagent. 
$\underline{\text { Page }}$

B. Hydroxylation with a Cyclodextrin-Modified

Hydroxylating System ................ 49

1. Stabilizing Effect of Cyclodextrins. ... 49

2. Effect of $\mathrm{pH}$ and Buffer Concentration. - 60

3. Analytical Results. ............. 65

c. Hydroxylation with a Glucose-Modified

Hydroxylating System ................ 73

1. Stabilizing Effect of Glucose. ...... 73

2. Temperature Effects. $\ldots \ldots \ldots \ldots \ldots \ldots .76$

3. Dependence on Catechol. ........... 81

4. Analytical Results. ............ 85

5. Other stabilizers. ............ 88

D. Studies on the Analytical Finish ......... 91

1. Stability of the 4-AminoantipyrineProduced Color. .................. 91

2. Other Finishes. $\ldots \ldots \ldots \ldots \ldots \ldots \ldots \ldots \ldots 96$

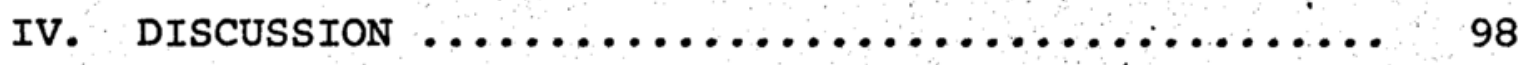

A. The Unmodified Hamilton System ......... 98

B. The Cyclodextrin Effect ............. 102

1. Cyclodextrin Inclusion Complexes. ..... 102

2. Hypothesis for Stabilization by

Cyclodextrins. .................. 107

C. General Stabilization Effects ........... 110

1. Kinetic Schemes. ................. 110

2. Comparison of Stabilizers. ........... I21 


\section{TABLE OF CONTENTS - cont.}

Page

D. Analysis by Hydroxylation ............. 121

1. Proposed Method. ................ 121

2. Range of Applicability. ........... 123

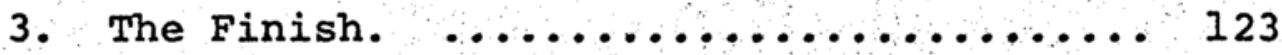

4. Comments on Product Yield and Distribution. .................. 125

5. Mixtures and Interferences. ......... 126

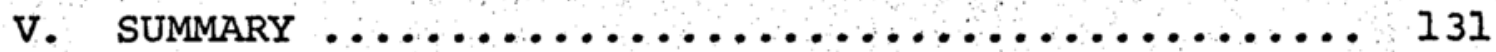

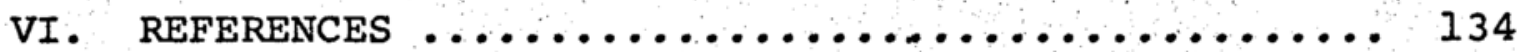

VII. APPENDICES $\ldots \ldots \ldots \ldots \ldots \ldots \ldots \ldots \ldots \ldots \ldots \ldots \ldots, 140$

A. Studies on the Analysis of Barbituric Acid Derivatives via Oxidation by Drug-

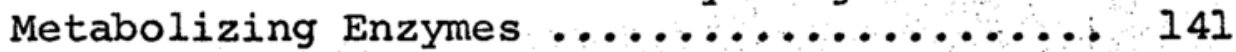

B. Determination of Hydroxy Compounds by Dimethylaminopyridine-Catalyzed 


\section{INTRODUCTION}

Enzymes have been widely applied as catalysts in the analysis of normal biochemical substances (1), but their potential utility as analytical reagents for the determination of drugs has not yet been extensively realized. The beginning of the present research was the idea that the selective determination of a drug might be accomplished by using as a catalytic reagent the very enzyme responsible for metabolizing the drug. This idea directed attention to the drug-metabolizing enzymes of the liver, in particular to the oxidases of the microsomal fraction (2). Appendix A reports preliminary experiments based on this approach, in which it was found that, although literature data on the oxidation of barbituric acid derivatives by rabbit liver microsomal fractions could be reproduced, the levels of enzymatic activity achieved were too low to justify further analytical development.

These studies did suggest, however, by analogy to mechanistic studies of enzyme action $(3,4,5)$, that a non-enzymatic model system might be found that would mimic some of the essential features of a drug-metabolizing oxidase. Many such systems have in fact been designed and studied for the insight they might provide into oxidase mechanisms. In the further development of this approach the available nonenzymatic aromatic hydroxylating systems were reviewed. Attention was restricted to the systems that function in an 
aqueous environment. The successful application of such a system as an analytical reagent could lead to a method for the analysis of aqueous solutions of aromatic compounds by their conversion to phenols, which would subsequently be determined by known methods.

\section{A. Aromatic Hydroxylation Systems}

Hydroxylation of aromatic compounds occurs readily in nature, and a number of non-enzymatic chemical systems that bring about this reaction in aqueous solution have been studied. These are listed in Table I. Reviews of aromatic hydroxylation are available $(6-10)$. of the several hydroxylation systems tabulated, those of Fenton (11-15), Hamilton $(16-20)$, and Udenfriend $(21-25)$ have been studied extensively.

In 1954, Udenfriend and co-workers (29) reported that aromatic compounds are hydroxylated by molecular oxygen or hydrogen peroxide when ferrous ion, ascorbic acid, and ethylenediamine are present in aqueous solution. These early investigators concluded that the product distribution (and hence mechanism) was the same when either $\mathrm{O}_{2}$ or $\mathrm{H}_{2} \mathrm{O}_{2}$ was used as an oxidant. More recent data $(15,24)$ have shown that different products are obtained with $\mathrm{H}_{2} \mathrm{O}_{2}$ and $\mathrm{O}_{2}$, and there is strong evidence that the hydroxyl radical is the actual hydroxylating agent when $\mathrm{H}_{2} \mathrm{O}_{2}$ is used as oxidizing agent (15). Table II, from Norman and Smith (7), compares product distribution for the oxygen mediated hydroxylation of monosubstituted benzene derivatives in the presence and absence 
Table I

Aqueous Model Enzyme Hydroxylation Systems

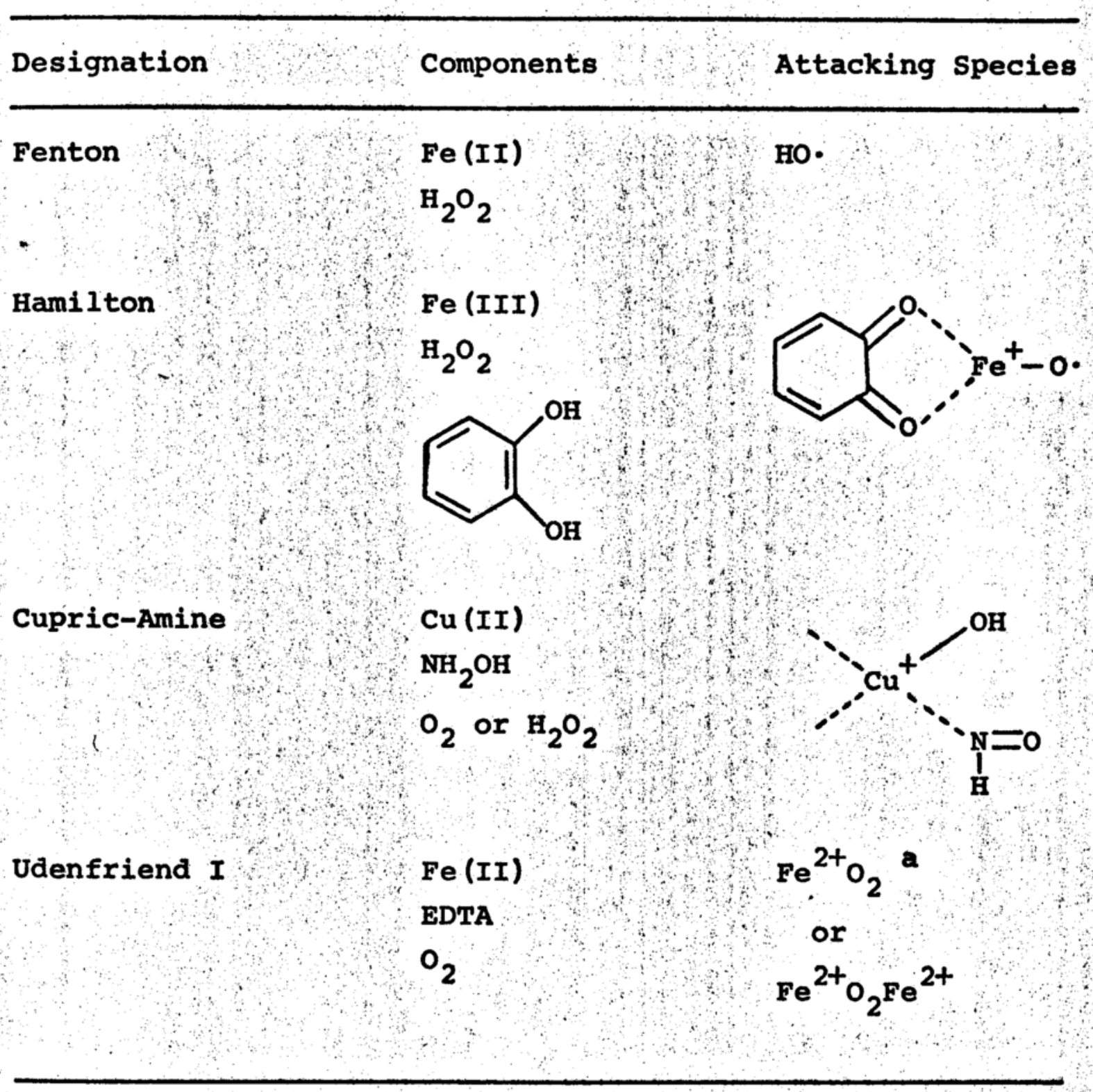

a Nature of attacking species dependent upon $\left[\mathrm{Fe}^{2+}\right]$. 
Table I - cont.

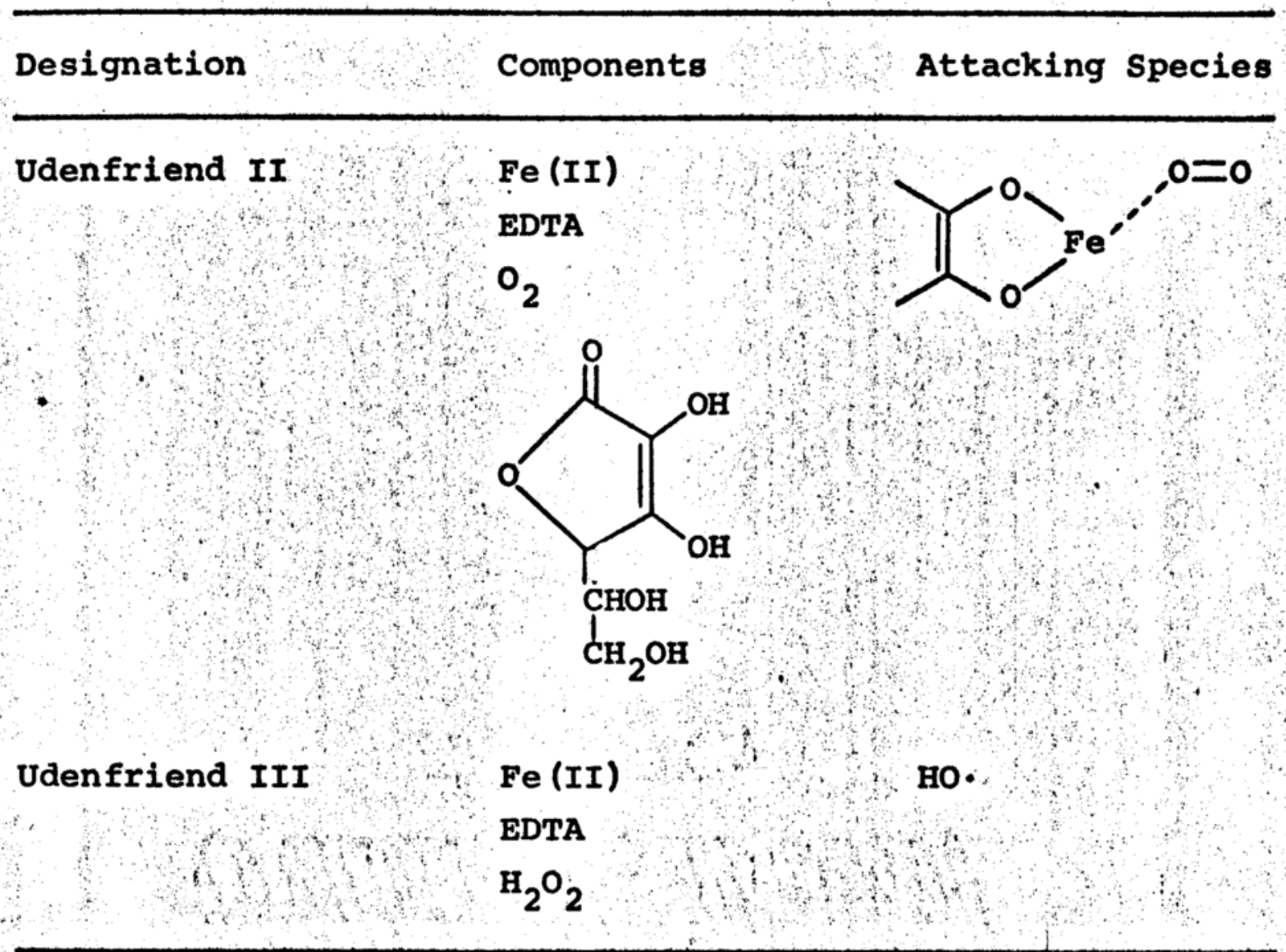


Table II

Isomer Distribution for the Hydroxylation of Substituted Aromatics by the Udenfriend System in the Presence and Absence of Ascorbic Acid (Norman and Smith ${ }^{7}$ )

Isomer Distribution (\%)

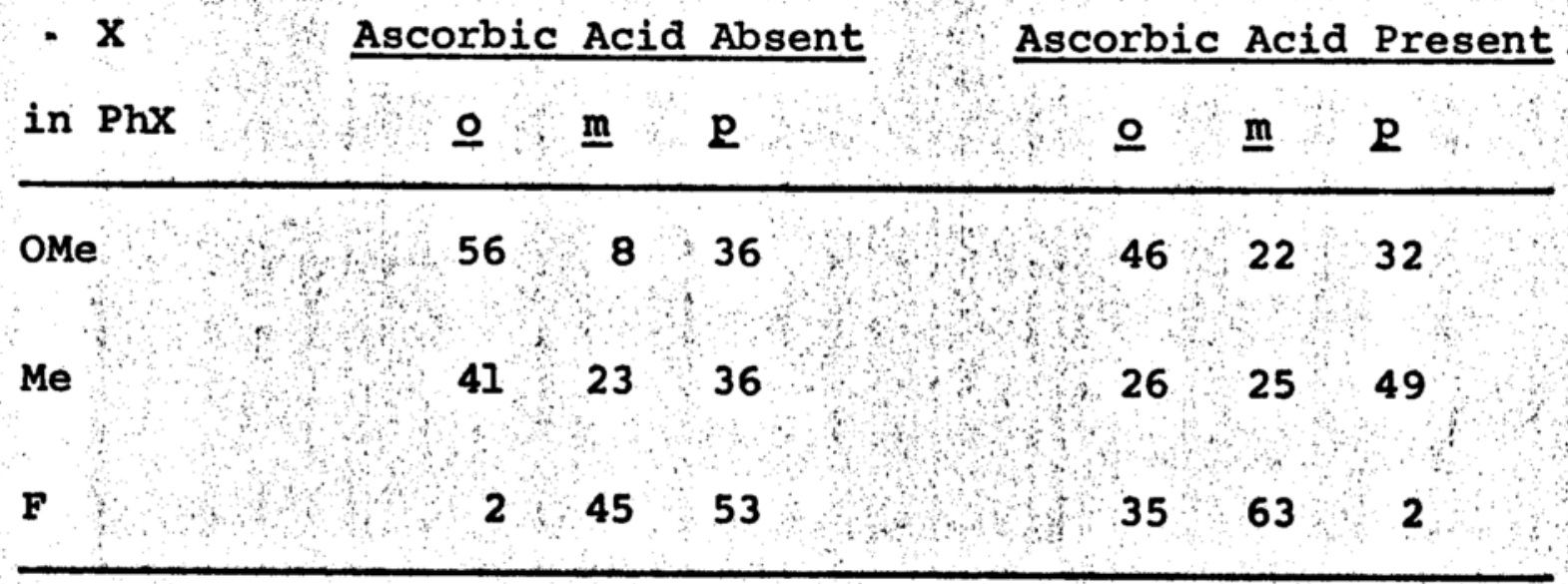


of ascorbic acid. It is apparent that different mechanisms must be involved because of the profound differences in isomer distribution. An Fe(II)-oxygen complex has been implicated in the Udenfriend scheme whether ascorbic acid is present or absent, the difference probably being due to the nature of the attacking species. Apparently, a complex of $\mathrm{O}_{2}$, ascorbic acid, and $\mathrm{Fe}(\mathrm{II})$ is derived by reaction of $\mathrm{Fe}^{2+} \mathrm{O}_{2}$ with ascorbic acid $(7,25)$.

Inspection of Table I reveals a marked similarity between the Udenfriend III and Fenton systems, the difference being only the presence of EDTA in the former. The following chain mechanism is the presently accepted scheme for the production of the hydroxylating agent in both these systems:

$$
\begin{aligned}
& \mathrm{Fe}^{2+}+\mathrm{H}_{2} \mathrm{O}_{2} \stackrel{\mathrm{k}_{\mathrm{I}}}{\longrightarrow} \mathrm{Fe}^{3+}+\mathrm{OH}^{-}+\mathrm{HO} \\
& \mathrm{Fe}^{2+}+\mathrm{HO} \stackrel{\mathrm{k}_{2}}{\longrightarrow} \mathrm{Fe}^{3+}+\mathrm{HO}^{-} \\
& \mathrm{HO}+\mathrm{H}_{2} \mathrm{O}_{2} \stackrel{\mathrm{k}_{3}}{\longrightarrow} \mathrm{HO}_{2}+\mathrm{H}_{2} \mathrm{O}
\end{aligned}
$$

Here, Eqs. (1) and (2) are chain initiation and termination steps, respectively, and Eq. (3) is a likely side reaction. There is some question whether the hydroxylating species is HO - or $\mathrm{HO}_{2} \cdot$ Evidence for the former has been supplied by Norman and co-workers (13), but Brodskii and Vysotskaya (14) have implicated $\mathrm{HO}_{2} \cdot$ by observing that a phenol derived from oxygen labeled hydrogen peroxide retained $95 \%$ of the labeled 
oxygen. If HO- were involved, rapid exchange with water would be expected. More recent kinetic evidence (26) suggests that the initial condition may determine the nature of the attacking species. since chain propagation occurs according to Eq. (4),

$$
\mathrm{HO}+\mathrm{ArH} \stackrel{\mathrm{k}_{4}}{\longrightarrow} \mathrm{H}_{2} \mathrm{O}+\mathrm{Ar} \cdot \frac{\mathrm{HO} \cdot}{\mathrm{k}_{5}} \mathrm{ArOH}
$$

and $k_{4} / k_{3}>10$, Eq. (3) can be made unimportant by maintaining a high ratio of $\frac{[\mathrm{ArH}]}{\left[\mathrm{H}_{2} \mathrm{O}_{2}\right]}$.

An alternative route for hydroxylation involving the hydroxyl radical (HO.) is described by Eq. (5).

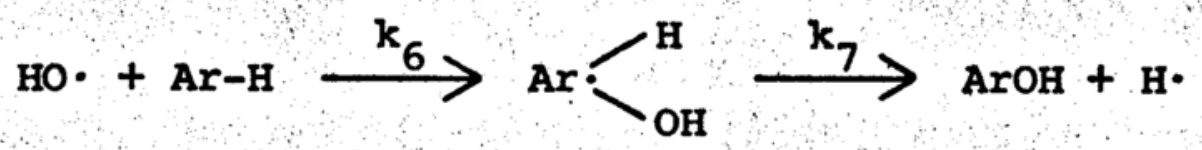

Norman and Smith (7) have supplied evidence for the preference of oxidation-elimination (Eq. 5) over hydrogen exchange (Eq. 4) by citing the existence of the hydroxyl-aryl radical by ESR spectroscopy.

The presence of EDTA has been shown to have no effect on the isomer distribution in the Fenton hydroxylation of aromatic compounds (15). EDTA increased the efficiency of hydroxylation, presumably by altering the oxidation potential of the ferrous-ferric couple (24). Further modification of the original Fenton system by adding ascorbic acid as well as EDTA has been described $(15,27)$. Since the product distribution was again unaltered, the nature of the attacking 
species was assumed unchanged.

Inspection of Table I reveals some striking similarities between the "modified" Fenton systems and those of Hamilton and Udenfriend. The Fenton system that incorporates EDTA, is, in fact, the Udenfriend III system. Further, the components of the Hamilton system bear a resemblance to the ascorbic acid-EDTA modified Fenton system and to the Udenfriend II system.

Hamilton $(18,19)$, in an attempt to find a version of the Udenfriend system better suited to the study of the mechanism of enzymic hydroxylations, replaced ascorbic acid by other enediols and looked for hydroxylation of anisole by hydrogen peroxide. He found that with catechol or hydroquinone as catalyst, a hydroxylating agent different from that in either of the Udenfriend reactions $\left(\mathrm{O}_{2}\right.$ or $\left.\mathrm{H}_{2} \mathrm{O}_{2}\right)$ was involved.

Hamilton, et al. $(18,19)$, studied the hydroxylation of some mono-substituted benzene derivatives, measuring the kinetics of loss of peroxide when the aromatic substrate was in excess. Table III compares the isomer distributions of phenols formed by the reaction of the Hamilton and Fenton reagents with a number of mono-substituted aromatic compounds, and includes the reactivity of these benzene derivatives in both systems. It seems clear from comparative isomer distributions and reactivities that the free hydroxyl radical (HO-) of the Fenton system is not the hydroxylating agent in the Hamilton system. The low intramolecular 
Table III

Product Distribution and Reactivity for the Hydroxylation of Aromatic Compounds by the Hamilton and Fenton Systems (Hamilton, et al. ${ }^{19}$ )

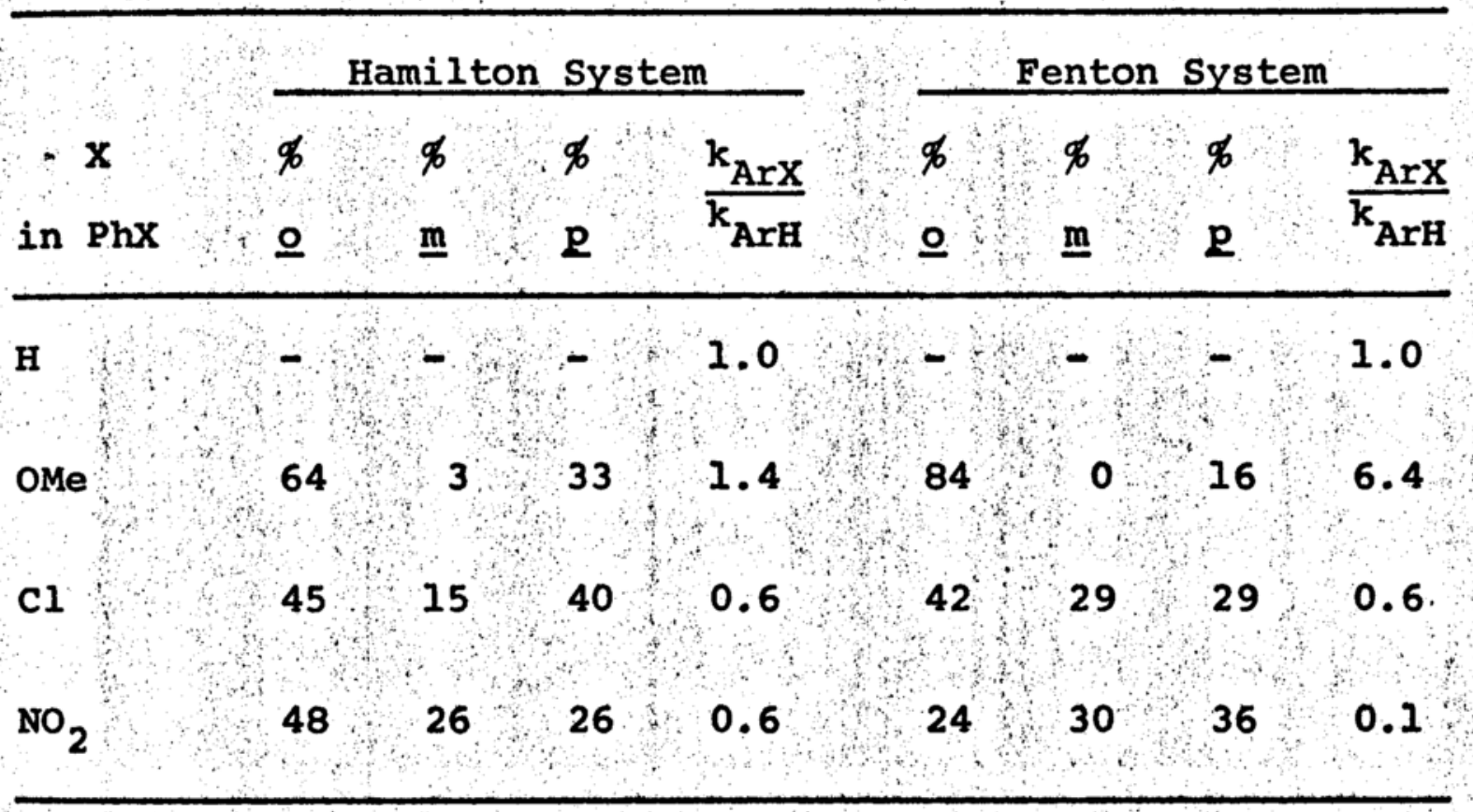


selectivity indicates a radical-like reagent, and the low substrate selectivity (anisole and nitrobenzene possess comparable reactivities) suggests a non-electrophilic reagent. In contrast, the Fenton reagent is electrophilic in nature, as shown by its higher substrate discrimination $\left(\mathrm{k}_{\mathrm{OMe}} / \mathrm{k}_{\mathrm{NO}} \sim\right.$ 64). Other salient features of the Hamilton system follow:

(1) If $\mathrm{Fe}$ (III) is omitted, no $\mathrm{H}_{2} \mathrm{O}_{2}$ reacts;

(2) The reaction is first-order in $\mathrm{Fe}$ (III),

(3) If the enediol is omitted, $\mathrm{H}_{2} \mathrm{O}_{2}$ reacts more than an order of magnitude slower than when catalytic amounts are present;

(4) Catechol and hydroquinone are catalysts; resorcinol and ascorbic acid are not catalysts;

(5) Fe(III) can be replaced by $\mathrm{Fe}$ (II), or, less effectively, Cu(II);

(6) Oxygen cannot be substituted for hydrogen peroxide;

(7) Reaction is inhibited by EDTA.

Based upon these results, the following mechanistic scheme for hydroxylation was suggested:<smiles>Oc1ccccc1O</smiles>

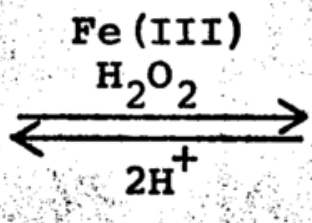<smiles>O[14C]Oc1ccccc1OO</smiles> 
<smiles>OC(O)COc1ccccc1O[P+]</smiles><smiles>O=[14C][14C](=O)[O-]</smiles><smiles>CO[P+](=O)Oc1ccccc1Oc1ccccc1</smiles><smiles>[O-][P+](O)Oc1ccccc1O</smiles><smiles>CCOc1ccccc1O[Po+]([O-])O</smiles>

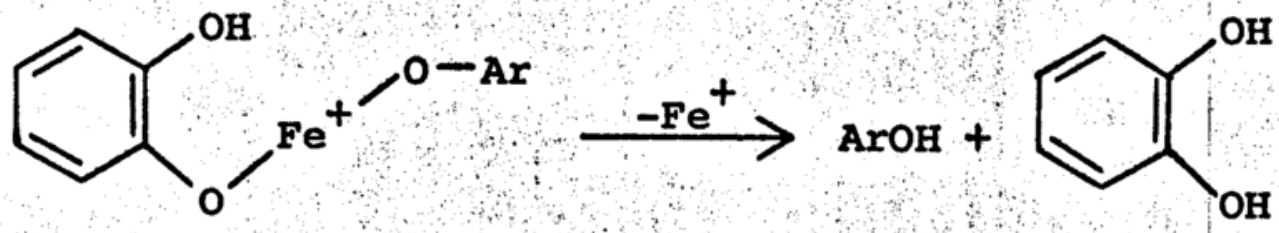

In the mechanism, the oxidizing agent is formed in a ratedetermining step (Eq. 7) by loss of a molecule of water from a complex of ferric ion, catechol, and hydrogen peroxide. Since iron can transfer electrons by overlapping its $\underline{a}$ orbitals with the $\mathfrak{p}$ orbitals of ligands (28), the transition state leading to the formation of the hydroxylating agent is resonance-stabilized. The scheme depicts reaction of the radical-like reagent with the aromatic compound (Eq. 8), which eventually leads to a phenol and regenerates the catalyst (Eq. 9). With compounds that are not catalysts, presumably the transition state of the rate-determining step 
is not stabilized sufficiently by resonance (resorcinol), or, once formed, is easily oxidized by addition of water across the iron-oxygen double bond (ascorbic acid).

From the foregoing discussion, the Hamilton hydroxylating system appears simpler than that of Udenfriend, and more rapid than the Fenton reagent. Further, the yields of hydroxylated product from the Hamilton hydroxylation (up to 50\%) are higher than those of either the Fenton or Udenfriend systems.

The ability of the Hamilton system to introduce a hydroxy group on an aromatic ring is, analytically, potentially useful. The product phenol may be determined by a number of physical and chemical methods.

\section{B. Methods of Phenol Determination}

A large number of procedures have been developed for the determination of organic compounds containing the phenolic function. Extensive reviews are available $(29,30)$. Table IV summarizes many of the methods that have been ' proposed.

As a final analytical measurement for phenols derived from the proposed aqueous hydroxylation of aromatic compounds, colorimetry offers the advantages of simplicity (color development occurs directly in aqueous solution) and speed (many of the common reactions are very fast). of the several colorimetric methods listed in Table IV, oxidative coupling suggests itself as applicable in the present 
Table IV

Summary of Methods for the Determination of Phenolic Compounds (Cheronis and $\mathrm{Ma}^{30}$ )

\section{Limit of Detection}

Method (amount or concentration)

\section{Titrimetric}

Halogenation

$0.1 \mathrm{meq}$

Nonaqueous titrimetry

Oxidation

Nitrosation

$0.1 \mathrm{meq}$

Esterification

$0.1 \mathrm{meq}$

$1 \mathrm{meq}$

1 meq

Gravimetric $^{a}$

1 meq

Gasometric

Grignard reagent

Lithium aluminum hydride

$0.1 \mathrm{meq}$

Coupling and nitrometry

$0.1 \mathrm{meq}$

$500 \mu g$

\section{Colorimetric}

Quinone chloramide ${ }^{b}$

4-Aminoantipyrine ${ }^{b}$

Diphenylpicrylhydrazil ${ }^{\mathrm{b}}$

3-Methyl-2-benzothiazolinone hydrazonec

N, N-Dimethy1-p-phenylenediamine

$$
\begin{aligned}
& 10^{-5} \underline{M} \\
& 10^{-5} \underline{M} \\
& 10^{-4} \underline{M} \\
& 10^{-5} \underline{M} \\
& 10^{-6} \underline{M}
\end{aligned}
$$


Table IV - cont.

Limit of Detection

Method (amount or concentration)

Colorimetric - cont.

Diazonium salt ${ }^{\mathrm{b}}$

1-Nitroso-2-naphthol

p-Aminodiethylaniline + oxidant

Phosphomolybdic acid

Xanthydrol

Nitrous acid

Sodium cupribromide

Fluorometric $^{\mathbf{b}}$

\section{Spectrophotometric}

IR

$$
\begin{aligned}
& 10^{-3} \underline{M} \\
& 10^{-4} \underline{M}
\end{aligned}
$$

Gas Chromatography

$1 \mathrm{ppb}$
a Precipitated as its 2,4-dinitrophenyl ether:
b s. Siggia (33).
C H. O. Friestad (32). 
instance because of the similar environments in the hydroxy1ation and coupling steps. This process was first developed with p-phenylenediamines, which upon oxidation in the presence of phenols, couple to give indaniline dyes. The reaction with phenol is shown in Eq. (10).<smiles>CN(C)c1ccc(N=C2C=CC(=O)C=C2)cc1</smiles>

The method has recently been re-investigated and optimum conditions proposed (31). Reaction of phenol with 3-methyl-2benzothiazolinone hydrazone, (MBTH), Eq. (11), forms the basis of an automated analytical method for the micro determination of phenols (32).

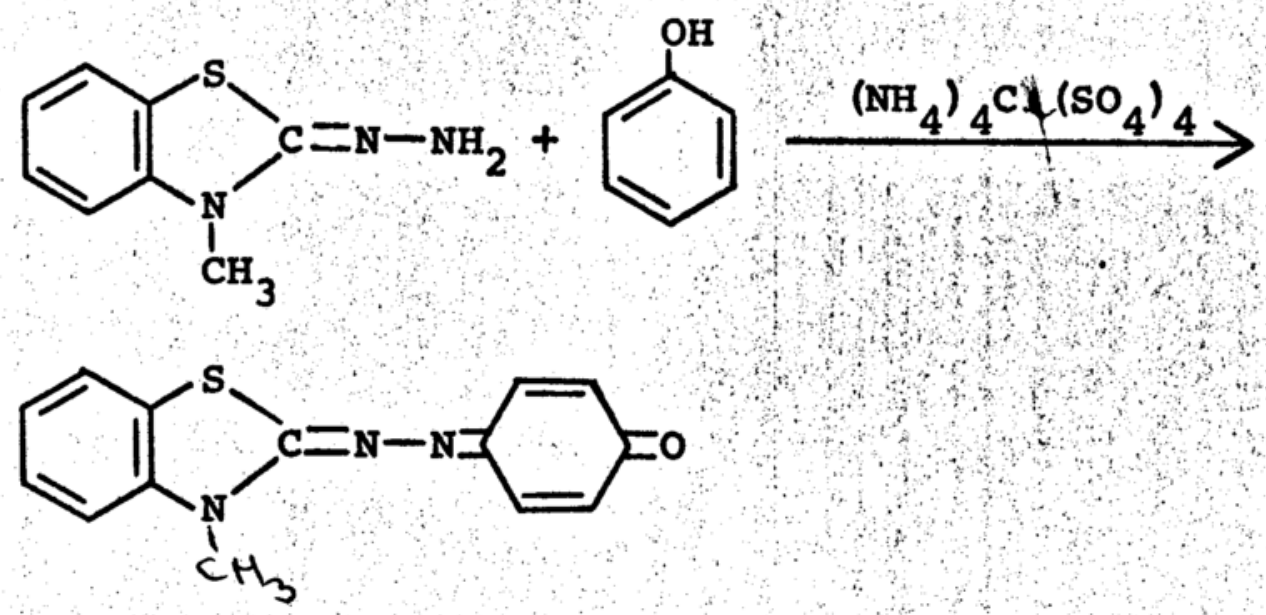

The formation of antipyrine dyes as a result of oxidative coupling of 4-aminoantipyrine (4-AAP) with phenols in the presence of an alkaline oxidizing agent (Eq. 12) has been used extensively to detect trace amounts of phenols $(33-39)$. 

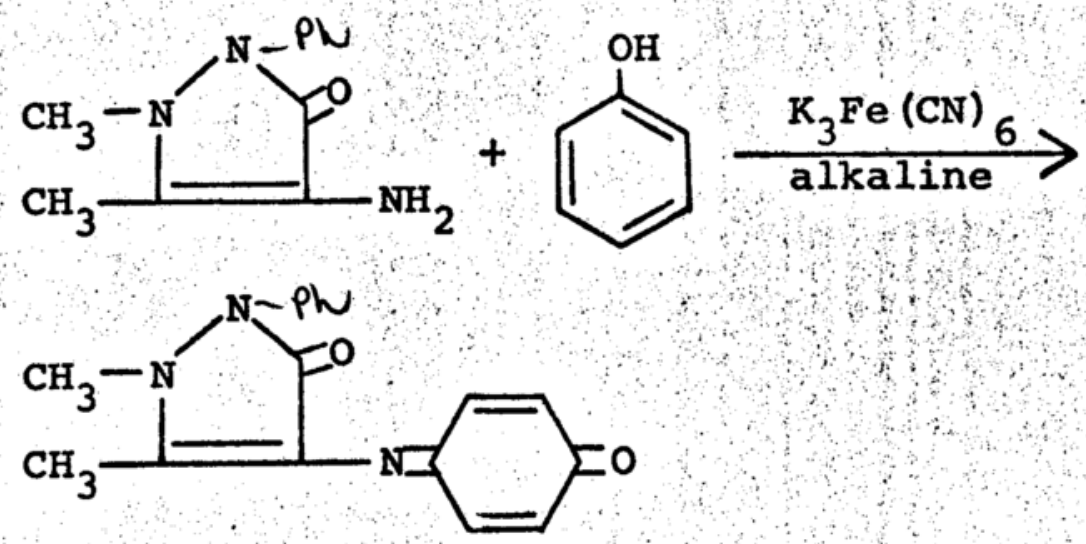

The Gibbs test, based on alkaline coupling of phenols with 2,6-dibromoquinone chloramide $(40-42)$, may be considered an oxidative coupling reaction (Eq. 13). Guilbault, et al. (43), employed a kinetic technique to eliminate the long reaction times involved in the usual Gibbs reaction.

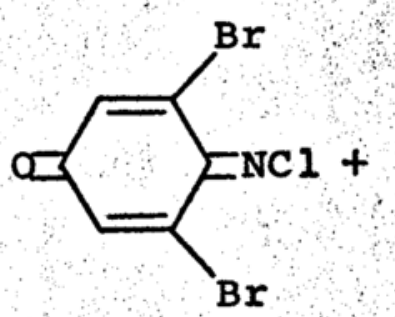<smiles>[O-]c1ccccc1</smiles>

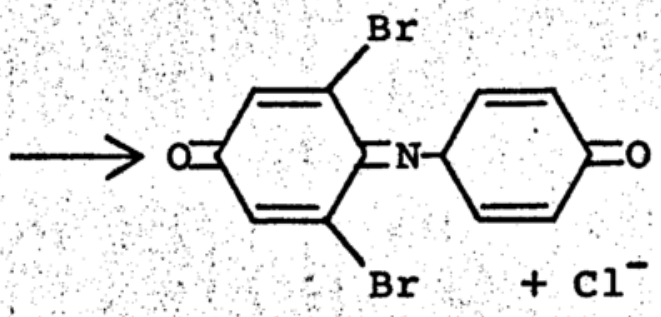

The Gibbs reagent appears to be the most specific of the oxidative coupling reagents, responding only to phenols unsubstituted in the para-position (40). Recently, however, Dacre (44) has shown that para-substituted alkoxyphenols respond, and that some $\mathrm{p}$-halogeno-phenols give positive tests, and finally, that many highly substituted phenols with the para-position unsubstituted, do not give a positive test. Mohler and Jacob (45) compared this method with the 4-AAP method and decided the latter was faster, more accurate, and more precise. 
Both the 4-AAP and phenylenediamine reactions are applicable to 2- and 3-substituted phenols as well as 4-. substituted phenols in which the para-position is not blocked by alkyl, nitro, benzoyl, or carbonyl groups $(31,39)$. The molar absorptivities for phenol are comparable.

Friestad, et al. (32), compared the 4-AAP and MBTH methods and found that the latter was less dependent on the position of substituent groups, and more sensitive. Table V contrasts the response of some selected mono-substituted phenols to both methods and illustrates the non-specificity and greater sensitivity of the MBTH method.

A reaction closely related to oxidative coupling is azo dye formation, illustrated in Eq. (14).

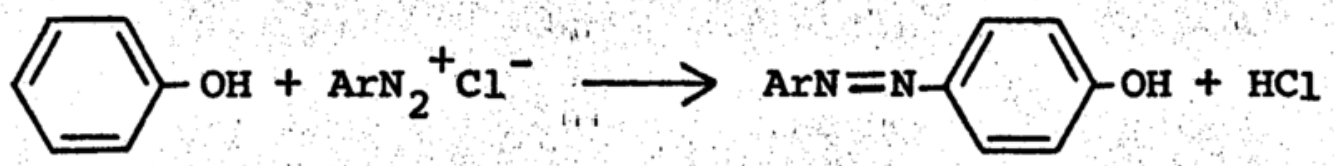

Diazo coupling, with the formation of a highly colored dye, is one of the most generally applicable methods for the determination of trace quantities of phenols $(33,46)$. The two aromatic amines most commonly used to form the diazonium salt are sulfanilic acid and p-nitroaniline. Coupling normally takes place in the para position of the phenol unless the position is blocked; then coupling occurs at the ortho position, but probably at a slower rate. If both ortho and para positions are occupied, no reaction occurs. Molar absorptivities obtained by this method are comparable with those for the MBTH reaction. 


\section{Table v}

Comparison of Molar Absorptivities for some Mono-substituted Phenols by Two Colorimetric Methods ${ }^{32}$

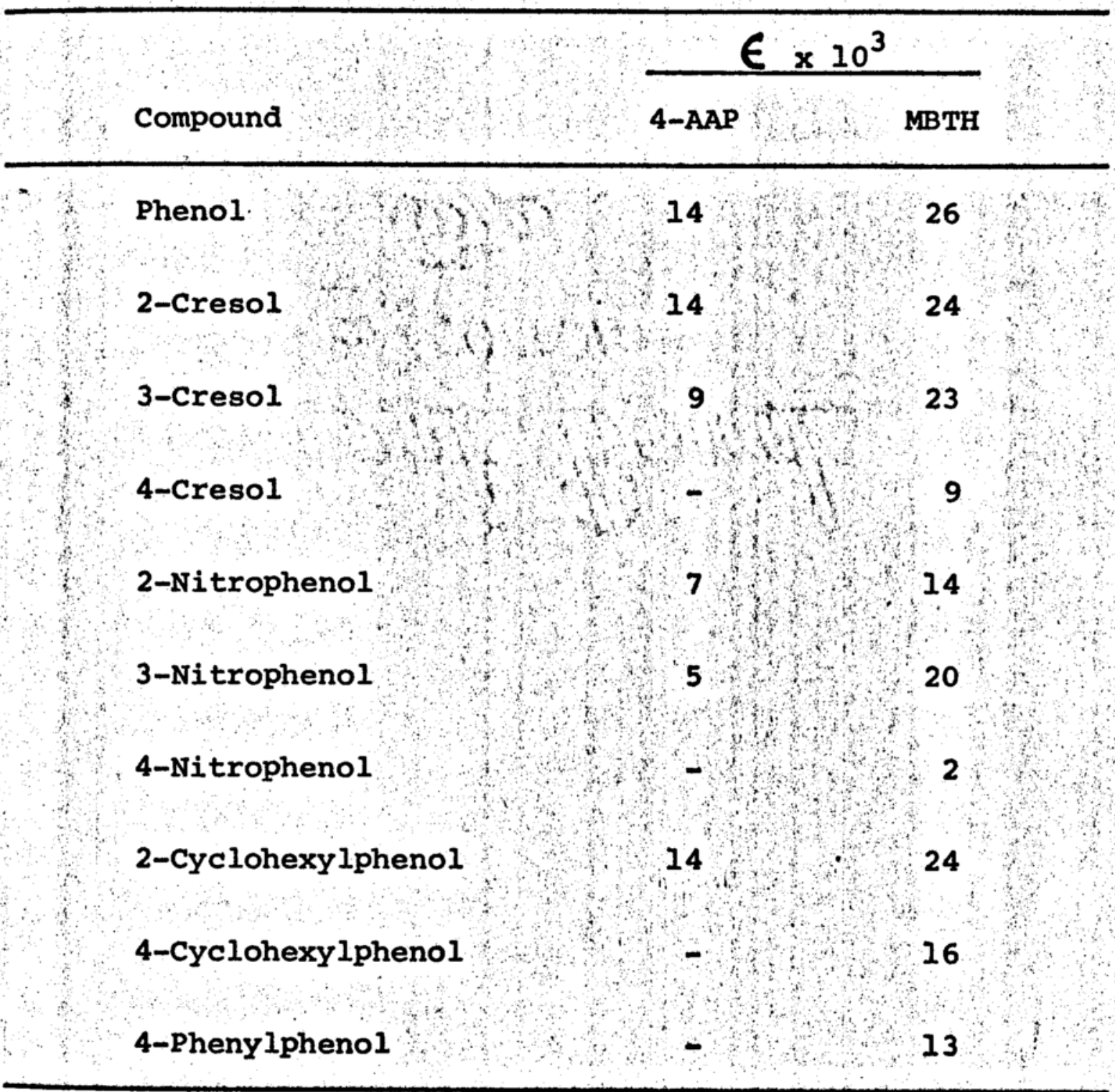


C. Research Plan

One of the fruitful directions of organic analytical research is the development of a body of general reactions upon which the analyst can draw when confronted with a particular problem. In this context, aromatic hydroxylation offers the possibility of a general approach for the detection of the phenyl group and the determination of aromatic compounds in aqueous solution.

Little prior use has been made of aromatic hydroxylation. The partial conversion of benzoic acid into salicylic acid by hydrogen peroxide was first observed by Hanriot (47), and the reaction formed the basis of a spot test for benzoic acid (48). Nicholls (49) observed that the proportion of benzoic acid converted to salicylic acid was a constant (12\%), and the method was modified for the determination of benzoic acid in food using a reagent of ferric ion, hydrogen peroxide, and sulfuric acid ${ }^{l}(50,51)$; the salicylic acid was removed by extraction and determined colorimetrically. Bartos (52) determined several aromatic compounds colorimetrically through formation of o-nitrosophenols using the cupric ion-hydrogen peroxide-hydroxylamine reagent (Table I). He subjected only five mono-substituted benzene derivatives to his method and observed deviations from Beer's Law for one of them. His method is not highly sensitive, requiring a minimum concentration of aromatic of $10^{-3} \mathrm{M}$.

1 This reagent is apparently the Fenton reagent. 
As previously noted, the Hamilton hydroxylating agent seems to provide advantages of simplicity, yield, and speed compared with the other systems listed in Table I. If the hydroxylation reaction occurs on a reasonable time scale and is general for aromatics of different substitution pattern and electronic configuration, than the Hamilton system may, provide a widely applicable method for generating phenols from aromatic compounds for analytical use.

The choice of colorimetric "finish" for subsequent phenol determination must be based upon sensitivity as well as possible interference caused by components of the Hamilton system. Since catechol, the enediol catalyst, is itself a phenol, it would be expected to respond to many of the general colorimetric methods for phenol determination. In this context, Lacoste (36) has observed that the 4-aminoantipyrine method is much less sensitive to catechol than to monohydric phenols. Because it would be desirable to employ the final analytical measurement in situ, the 4-AAP method may be the finish of choice.

The plan of the analytical method is illustrated by Eq. (15).<smiles>[R]c1ccccc1</smiles>

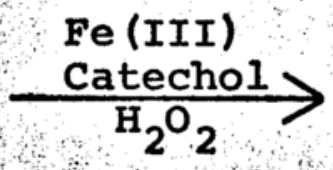<smiles>[R]c1cccc(O)c1</smiles><smiles>[R]CC(=O)c1c(C)n(C)n(-c2ccccc2)c1=O</smiles> 
An aqueous aromatic sample is hydroxylated by the Hamilton system; the corresponding phenol is subsequently determined by oxidative coupling with 4-aminoantipyrine. As one of the few methods available for the detection of the phenyl group ${ }^{2}$, this technique would have the advantages and limitations of a general functional group method.

2 Nitration and bromination have found limited use $(30,50)$. 


\section{EXPERIMENTAI}

\section{A. Materials}

All inorganic chemicals were analytical reagent grade, unless otherwise stated, and were used without further purification. Ferric perchlorate hexahydrate $\left(\mathrm{Fe}\left(\mathrm{ClO}_{4}\right)_{2} \cdot 6 \mathrm{H}_{2} \mathrm{O}\right)$ was obtained from G. F. Smith Co. and was used directly. Both ceric ammonium sulfate and potassium ferricyanide were obtained from J. T. Baker Chemical Co.

The following chemicals, listed according to source. were analytical reagent grade, and were used without further purification: Mallinckrodt (ethyl acetate, acetone, chloroform, methanol, benzene, toluene, isopropyl alcohol, benzoic acid and phenol); J. T. Baker Chemical Co. (anhydrous dextrose, aniline, and hydrogen peroxide - 30\%); Merck Chemical Co. (pyridine). Hydrogen peroxide solutions ( $3 \%$ - prepared by diluting the $30 \%$ solution) were standardized against permanganate.

Sorbitol, acetonitrile, and 1,4-dioxane were all used as received (Fisher scientific Co.. certified reagent). The following chemicals (J. T. Baker Chemical Co.) were used without further purification: lactose (U.S.P.), stearic acid (U.S.P.), benzaldehyde (N.F.), nitrobenzene (Purified), and hydroquinone (Purified). Talc-UsP (Merck) and corn starch (Beaver Dam Wholesale Co.) were also used directly. The following compounds were all used without further purification: N,N-dimethyl-p-phenylenediamine hydrochloride 
(Eastman White Label), 4-aminoantipyrine (Aldrich Chemical Co.). 3-methy1-2-benzothiazolinone hydrazone (Aldrich Chemical Co.), N,N-dimethylformamide (Eastman White Label), triethylenediamine (Aldrich Chemical Co.), Schardinger $\alpha$ - and B-dextrin (Pierce Chemical Co.). I-phenylalanine (Schwartz/. Mann). I-tryptophan (Nutritional Biochemical Corp.), sodium pentobarbital ( $\mathrm{K}$ and $\mathrm{K}$ Laboratories, Inc.), quinine sulfate (Fisher Scientific Co.), Zephiran chloride, 1:750 (Winthrop Labs), salicylamide (Eastman White Label), 3-methyl catechol and 4-methyl catechol (Aldrich Chemical Co.), and 2-, 3-, and 4-hydroxypyridines (Aldrich Chemical Co.). Physostigmine sulfate (commercial source), was also used directly.

Catechol (Matheson, Coleman and Beli) was recrystallized twice from toluene, m.p. $103-104^{\circ}$ [1it. (54), $104^{\circ}$ ]; hydrocinnamic acid (Eastman) was recrystallized from petroleum ether, m.p. $47-48^{\circ}$ [1it. (55), 47.5-48 ${ }^{\circ}$; benzamide (Eastman) was purified by recrystallization from water, m.p. 125.5-127 [lit. (56), $125-126^{\circ}$ ]; the isomers of methoxybenzoic acid were all recrystallized from water; ㅇ-methoxybenzoic acid, m.p. 98.5-100 [lit. (56), 99-101 ${ }^{\circ}$ ], m-methoxybenzoic acid, m.p. $104.5-105^{\circ}$ [1it. (56), 104-105], p-methoxybenzoic acid, m. p. $183-184^{\circ}$ [1it. (57), $\left.184^{\circ}\right]$.

N-methylbenzylamine (Eastman) was distilled at reduced pressure, b.p. $71 \% / 2 \mathrm{~mm}$ [lit. (58), b.p. $184^{\circ} / 760 \mathrm{~mm}, 78^{\circ} / 14$ mm]; methyl benzoate (Eastman) was purified by distillation, b.p. $199.6^{\circ}$ [lit. (59), b.p. 200\%]; ethyl benzene (Eastman) was distilled, b.p. $135^{\circ}$ [lit. (60), b.p. $135^{\circ}$ ]; benzyl 
alcohol (Eastman) was distilled at reduced pressure, b.p. $70 \% / 15 \mathrm{~mm}$ [lit. (61), $104-105 \% / 20 \mathrm{~mm}$ ]

Salicylic acid (Eastman) was recrystallized from methanol/water, m.p. $159-160^{\circ}$ [lit. (62), 158 ${ }^{\circ}$; m-hydroxybenzoic acid (Eastman) was purified by recrystallizing from water, m.p. $200^{\circ}$ [lit. (63), $202^{\circ}$ ]; p-hydroxybenzoic acid (Eastman) was recrystallized from xylene/ethanol, m.p. 213$214^{\circ}\left[1\right.$ it. $\left.(64), 212-213^{\circ}\right]$.

o-Cresol (Eastmán) was distilled under reduced pressure, b.p. $191-192^{\circ} / 760 \mathrm{~mm}\left[1 i t .(65), 192^{\circ}\right] ;$ m-cresol (Eastman White Label), and p-cresol (Eastman White Label, b.p., 90\%/11 mm) [lit. (66), 95-96\% $/ 15 \mathrm{~mm}$ ] were used without further purification.

o-Aminophenol and $\underline{m}$-aminophenol were both recrystallized from toluene/methanol, m.p. $174.5-176^{\circ}$ and $122-123^{\circ}$, respectively [lit. $(67,68), 171^{\circ}, 123^{\circ}$ respectively].

Guaiacol (Eastman) was distilled under reduced pressure, m.p. $28^{\circ}$ [lit. (69), $27.4^{\circ}$ ] ; p-methoxyphenol (Eastman) was also purified by distilling under reduced pressure, m.p. $53^{\circ}$ [lit. (70), $53^{\circ}$ ].

ㅇ-Nitrophenol (Eastman) was recrystallized from absolute ethanol, m.p. $44.5-45^{\circ}$ [lit. (71), 45 $]$; m-nitrophenol (Eastman) was purified by recrystallizing from aqueous $\mathrm{HCl}$, m.p. 95-96 ${ }^{\circ}$ [1it. (72), 95-96 ${ }^{\circ}$; p-nitrophenol was recrystallized from toluene, m.p. $113-114^{\circ}\left[1\right.$ it. (73), $\left.114^{\circ}\right]$.

Salicylaldehyde (Eastman) was purified by distillation at reduced pressure, b.p. $65-66 \% / 2 \mathrm{~mm}$ [1it. (74), $197 \% / 760 \mathrm{~mm}$ ] ; 
salicylhydrazide was used as received; m-chlorophenol (Eastman) was used without further purification, b.p. $214^{\circ}$ [1it. (75), 213-216 ${ }^{\circ}$, as was m-hydroxyacetophenone (Aldrich), m.p. 94-96 ${ }^{\circ}$ [1it. (76), $96^{\circ}$ ]; however, m-hydroxybenzaldehyde (Eastman) was purified by recrystallizing twice from water, m.p. 101-1030 [1it. $\left.(7,7), 101-102^{\circ}\right]$.

Anisole (Aldrich Chemical Co.) was found to be gas chromatographically pure $\left(10 \% \mathrm{SE}-30,165^{\circ} \mathrm{C}\right)$.

The following drugs were used without further purification: chloramphenicol (Ruger Chemical Co.), m.p. 150-151.5 ${ }^{\circ}$ [lit. (78), 150.5-151.5 ${ }^{\circ}$; benzocaine (Matheson, Coleman and Bell), m.p. 87.5-89.0 [1it. (79), 90 $]$; phenacetin (Ruger, Chemical Co.), m.p. $134-135^{\circ}$ [lit. (80), $134^{\circ}$ ], dl-mandelic

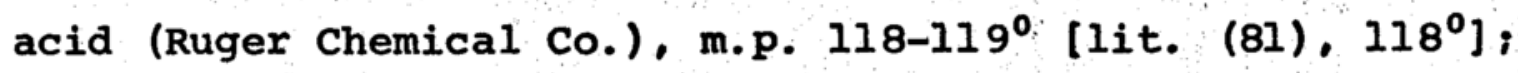
antipyrine (Ruger Chemical Co.), m.p. 109-110 $0^{\circ}$ [1it. (82), 109.5-110.5 ${ }^{\circ}$; dl-ephedrine hydrochloride (Merck), m.p. 187$188^{\circ}$ [lit. (83), 187.5-189.5 ${ }^{\circ}$; benzenesulfonamide (Aldrich), m.p. $152-154^{\circ}$ [lit. (84), $154^{\circ}$ ]; sulfanilamide (Merck), m.p. 163.5-164.5 [lit. (85), $166.5^{\circ}$ ].

Atropine sulfate, m.p. $188-190^{\circ}$ [lit. (86), $191^{\circ}$ ] and chlorpheniramine maleate, m.p. $135-136^{\circ}$ [1it. (87), $135^{\circ}$ ], were obtained from commercial sources and used without further purification.

Acetanilid (Merck) was purified by recrystallization from water, m.p. $113-114^{\circ}$ [lit. (88), $114^{\circ}$ ]; acetylsalicylic acid (Merck) was recrystallized from acetone, m.p. 135-136 [lit. (89), $135^{\circ}$ ], 2-naphthalene sulfonic acid (Matheson, 
Coleman and Bell) was recrystallized from 20\% dioxane in benzene, m.p. $121-123^{\circ}$ [lit. (90), 120-122 ${ }^{\circ}$; phenobarbital (z. D. Gilman, Inc.) was recrystallized from water/methanol, m.p. $174-176^{\circ}$ [lit. (91), $175.5^{\circ}$ ]; barbital (z. D. Gilman, Inc.) was recrystallized from water, m.p. $188-189.5^{\circ}$ [1it. (92), $190^{\circ}$ ]; dl-tropic acid, obtained from commercial sources, was further purified by recrystallization from benzene, m.p. $116-117^{\circ}$ [1it. (93), $116-117^{\circ}$ ].

Standard buffers were prepared according to Bates (94) using freshly boiled, purified water. Water was purified by passage of once-distilled water through an ion-exchange column (Continental Deionized Water Service). pH 4.0 acetate buffer, in which hydroxylation reactions were run, was prepared to be $5 \times 10^{-3} \underline{M}$ in total buffer concentration, ionic strength $0.1 \mathrm{M}$. Ionic strengths were adjusted using potassium chloride. Borate buffer, used to quench hydroxylations and to develop colors, was prepared to be $0.4 \mathrm{M}$ in total boric acid and $0.2 \underline{M}$ in sodium hydroxide. Aqueous catechol $(3 \times$ $10^{-3}$ M) was stored in the dark. Ferric perchlorate stock solution $\left(1.5 \times 10^{-2} \underline{M}\right)$ was stabilized by adding one drop of $60 \%$ perchloric acid per 50.0 cc of solution. Ferric perchlorate reagent solution $\left(1.5 \times 10^{-3} \mathrm{M}\right)$ was prepared by diluting $1.0 \mathrm{cc}$ of ferric perchlorate stock solution to 10.0 cc with water.

B. Apparatus

Spectral measurements were made with either a Cary model 
14 or model 16 spectrophotometer fitted with thermostated cell compartments that maintained temperature constant to $\pm 0.1^{0}$

$\mathrm{pH}$ measurements at $25.0^{\circ} \mathrm{C}$ were made with either a Radiometer pH meter model 25 with scale expander and equipped with a Corning combination electrode 476051 or a Sargent model DR expanded scale digital readout $\mathrm{pH}$ meter equipped with a Sargent combination electrode s-30072-15.

Water bath temperatures at $25.0^{\circ}$ were maintained to $\pm 0.1^{\circ}$ with a sargent Thermonitor Electronic Relay. Water bath temperatures at $50^{\circ}$ and $75^{\circ}$ were maintained to $\pm 0.1^{\circ}$ with a "Temptrol 151 " water bath (Precision Scientific Co.) or with a "Dual-Purpose" water bath system equipped with a relay, heater, stirrer and circulator (American Instrument Co.) and regulated by a mercury column thermoregulator (Branwill scientific Co.) ${ }^{1}$

Corrected melting points were determined on a ThomasHoover Capillary melting point apparatus.

Thermometers for use at $25.0^{\circ}$ were calibrated against a thermometer carrying a National Bureau of Standards calibration certificate. Thermometers for use at $50^{\circ}$ and $75^{\circ}$ were calibrated against a reference thermometer which had been calibrated against a thermometer carrying an ASTM certificate. Pre-coated thin layer plates, $0.25 \mathrm{~mm}$ thickness (Silica Gel G or Silica Gel F-254) were obtained from Brinkman

I I am indebted to Prof. J. R. Robinson for graciously loaning this high-temperature water bath. 
Instruments; pre-coated cellulose sheets (with and without fluorescent indicator) were obtained from Eastman Kodak. A spray kit, consisting of a reservoir bottle, sprayer head and propellant (Chromatosprayer) was obtained from Scientific Manufacturing Industries.

\section{c. Procedures}

\section{Hydroxylation Time Course Studies.}

Reaction mixtures were prepared in $50-\mathrm{ml}$ volumetric flasks by mixing appropriate volumes of reagent and substrate solutions, and adjusting pH between 3.5 and 4.0 with concentrated hydrochloric acid or saturated sodium hydroxide solution. After the solutions had been brought to volume with pH 4.0 acetate buffer, their temperatures were adjusted to the desired reaction conditions $\left(25^{\circ}, 54^{\circ}\right.$, or $\left.75^{\circ}\right)$ by placing them in a water bath. ${ }^{2}$ Typical concentrations in the final solution were $1 \times 10^{-4} \underline{M}$ aqueous catechol, $5 \times 10^{-5} \underline{M}$ ferric perchlorate, and between $10^{-3}$ and $10^{-4}$ M aromatic substrate. When a "stabilizer" was present, its initial concentration was chosen after preliminary experiments established an "effective" concentration (see Results Section).

Reactions were initiated by adding $0.2 \mathrm{ml}$ of $3 \%$ hydrogen peroxide solution to the $50 \mathrm{ml}$ reaction mixture. Samples were removed at recorded times by pipetting a $5.0 \mathrm{ml}$ aliquot

2 Elevated temperatures were needed to shorten the reaction time. 
into $5.0 \mathrm{ml}$ of borate buffer. ${ }^{3}$ This quenches the reaction. Colors were developed by adding $0.1 \mathrm{ml}$ of $3 \%(\mathrm{w} / \mathrm{v})$ aqueous 4-aminoantipyrine followed by $0.1 \mathrm{ml}$ of $10 \%(\mathrm{w} / \mathrm{v})$ aqueous potassium ferricyanide. 4 The absorbance was measured immediately at the absorption maximum against a reagent blank carried through the same procedure. 5 plots of absorbance against time are subsequently prepared.

\section{Proposed Analytical Method.}

To a $25 \mathrm{ml}$ volumetric flask are added $1.0 \mathrm{ml}$ of $3 \times 10^{-3} \underline{\mathrm{M}}$ aqueous catechol, $1.0 \mathrm{ml}$ of $1.5 \times 10^{-3} \underline{\mathrm{M}}$ ferric perchlorate, $5.0 \mathrm{ml}$ of $1.8 \times 10^{-2} \mathrm{M}$ glucose, and enough of the aromatic compound such that its concentration in the diluted solution will be in the range $10^{-4}$ to $10^{-3}$ M. The solution is diluted to the mark with $\mathrm{pH} 4.0$ acetate buffer. The $\mathrm{pH}$ of this reaction solution should be in the range of 3.5 to 4.0 .

The reaction vessel is placed in a $75^{\circ}$ water bath and reaction is initiated by adding $0.1 \mathrm{ml}$ of $3 \%$ hydrogen peroxide

3 A $2.0 \mathrm{ml}$ aliquot was pipetted into $20.0 \mathrm{ml}$ of borate buffer for experiments involving the hydroxylation of anisole with the Hamilton system (see Results Section).

4

In preliminary experiments, $0.2 \mathrm{ml}$ of potassium ferricyanide was used (see Discussion Section).

5

It has been shown (Ref. 37 and later portions of this thesis) that the red antipyrine dye is not very stable for phenols containing electron withdrawing groups, hence, the absorbance should be read immediately. When color fading occurs, absorbance values were extrapolated to time of color development (see Discussion Section). 
solution. After $10 \mathrm{~min}$. at $75^{\circ}$, a $5.0 \mathrm{ml}$ aliquot is pipetted into $5.0 \mathrm{ml}$ of borate buffer. The color is developed by adding $0.1 \mathrm{ml}$ of $3 \%(\mathrm{w} / \mathrm{v})$ aqueous 4-aminoantipyrine followed by $0.1 \mathrm{ml}$ of $10 \%(\mathrm{w} / \mathrm{v})$ aqueous potassium ferricyanide. The absorbance is measured immediately at the absorption maximum against a reagent blank carried through the same procedure. A standard curve is prepared by subjecting known concentrations, bracketing the unknown concentration, of the same aromatic compound to the procedure.

3. Studies on the Analytical Finish

(a) The 4-Aminoantipyrine Method. Aqueous stock solutions of monosubstituted phenols were prepared to be in the range 5 to $7 \times 10^{-3} \mathrm{M}$. These were further diluted with borate buffer such that colors produced by adding $0.1 \mathrm{ml}$ of $3 \%(\mathrm{w} / \mathrm{v})$ 4-aminoantipyrine followed by $0.2 \mathrm{ml}$ of $10 \%(\mathrm{w} / \mathrm{v})$ potassium ferricyanide to $25.0 \mathrm{ml}$ of solution, gave initial absorbance values between 0.3 and 0.9 absorbance units when measured at $510 \mathrm{~nm}$ against a reagent blank treated in the same way. Absorbances were recorded as a function of time in $1 \mathrm{~cm}$ cells on the Cary model 14 spectrophotometer. Normalized plots of absorbance vs time were subsequently prepared.

Some neutral phenols were subjected to a chloroform extraction using the following procedure: aqueous stock solutions of neutral mono-substituted phenols were diluted with borate buffer such that absorbances in the final 
chloroform colorimetric solution were in the range 0.4 to 1.0 absorbance units when measured at $485 \mathrm{~nm}$ against a reagent blank treated in the same manner. To a $10 \mathrm{ml}$ aliquot of phenol sample in borate buffer contained in a $60 \mathrm{ml}$ separatory funnel were added $0.1 \mathrm{ml}$ of $3 \%(\mathrm{w} / \mathrm{v})$ 4-aminoantipyrine and $0.1 \mathrm{ml}$ of $10 \%(\mathrm{w} / \mathrm{v})$ potassium ferricyanide. The aqueous phase was extracted with a $15 \mathrm{ml}$ and a $5 \mathrm{ml}$ portion of chloroform, the extracts being filtered through glass wool and combined in a $25 \mathrm{ml}$ volumetric flask. The extracts were diluted to volume with chloroform and the absorbance measured as a function of time in $1 \mathrm{~cm}$ cells at $485 \mathrm{~nm}$ against a reagent blank treated in the same manner. Normalized plots of absorbance against concentration were prepared.

Neutral aromatic compounds could be determined with increased sensitivity after hydroxylation by concentrating the final colored solution in chloroform, as above, with the following procedure: after hydroxylation was complete (see Results Section), $15.0 \mathrm{ml}$ of reaction mixture was pipetted into a $60 \mathrm{ml}$ separatory funnel containing $10 \mathrm{ml}$ of borate buffer. To this was added $0.1 \mathrm{ml}$ of $3 \%(\mathrm{w} / \mathrm{v})$ 4-aminoantipyrine, about $4 \mathrm{ml}$ of chloroform, followed by $0.1 \mathrm{ml}$ of $10 \%$ (w/v) potassium ferricyanide, and the mixture was shaken for about one minute. The chloroform layer was filtered through glass wool into a $5 \mathrm{ml-volumetric} \mathrm{flask.} \mathrm{The} \mathrm{separatory}$ funnel was rinsed with an additional $0.75 \mathrm{ml}$ of chloroform, which was combined with the first chloroform extract, and this solution was diluted to volume with chloroform. The 
absorbance was measured at the absorption maximum against a reagent blank carried through the same procedure.

(b) MBTH Method. Qualitative color tests were performed with phenol in the presence of components of the Hamilton system using oxidative coupling with 3-methyl-2benzothiazolinone hydrazone (MBTH).

To $1.0 \mathrm{ml}$ of aqueous phenol $(5 \mu \mathrm{g} / \mathrm{ml})$ containing combinations of the following: $1 \times 10^{-4} \underline{\mathrm{M}}$ catechol, $5 \times 10^{-5} \underline{\mathrm{M}}$ ferric perchlorate, $1 \times 10^{-3} \underline{M}$ hydrogen peroxide; was added $1.0 \mathrm{ml}$ of $0.05 \%(\mathrm{w} / \mathrm{v})$ aqueous MBTH. After $5 \mathrm{~min} .$, the color was developed by adding $1.0 \mathrm{ml}$ of $0.2 \%(\mathrm{w} / \mathrm{v})$ ceric ammonium sulfate in $0.4 \% \mathrm{H}_{2} \mathrm{SO}_{4}$.

\section{Attempted TLC Detection Studies.}

Stock solutions of mono-substituted aromatic and monosubstituted phenols were prepared to be $1 \mathrm{mg} / \mathrm{ml}$ in methanol. The following spray reagents were used:

$\underline{I} 2 \times 10^{-4} \underline{M}$ catechol and $1 \times 10^{-4} \underline{M}$ ferric perchlorate in $\mathrm{pH} 4.0$ acetate buffer.

II $3 \% \mathrm{H}_{2} \mathrm{O}_{2}$ in $\mathrm{pH} 4.0$ acetate buffer (prepared by diluting $30 \%$ hydrogen peroxide $1: 10$ with buffer).

- III $0.24 \%$ 4-aminoantipyrine in borate buffer.

IV $0.4 \%$ potassium ferricyanide in borate buffer.

Between 2 to $10 \mu \mathrm{g}$ of sample (aromatic or phenol) was applied to cellulose or silica gel plates using $2-\mu 1$ micropipettes (Drummond Microcaps). The sample spots were airdried and sprayed consecutively with sprays I and II (aromatic 
compounds only). After hydroxylation (about 10 min.) the plates were sprayed with Sprays III and IV for visualization. 
III. RESULTS

A. Hydroxylation of Anisole with the Hamilton System

A linear Beer's Law plot was observed for the determination of phenol by means of the 4-aminoantipyrine (4-AAP) coupling method in the presence of components of the Hamilton system. The molar absorptivity obtained $\left(1.2 \times 10^{4}\right)$ is close to literature values (see Table v). No interference by catechol was observed. This lack of interference by the Hamilton system made the 4-AAP method the finish of choice, especially for the early developmental studies.

\section{Dependence of Time Course Appearance on Peroxide/} substrate Ratio.

The experimental conditions employed by Hamilton $(17,18)$ include the following initial reactant concentrations: catechol, $1.5 \times 10^{-4} \underline{M}$ i Fe(III) $4 \times 10^{-5}$ M; $\mathrm{H}_{2} \mathrm{O}_{2}, 2 \times 10^{-3} \mathrm{M} ;$ anisole, $10^{-2}$ ‥ As an analytical reagent, however, the hydrogen peroxide in the Hamilton system must be in excess over the aromatic sample. The experimental conditions were therefore altered to reflect a more realistic system for the analysis of aromatic compounds. The following conditions were initially chosen: catechol, $1 \times 10^{-4} \mathrm{M} ; \mathrm{Fe}$ (III), $4 \mathrm{x}$ $10^{-5} \underline{\mathrm{M}} ; \mathrm{H}_{2} \mathrm{O}_{2}, 2 \times 10^{-2} \underline{\mathrm{M}}$; anisole, $5 \times 10^{-4} \underline{\mathrm{M}}$. Under these circumstances, low yields (less than 10\%) of phenol product were obtained after $2-1 / 2$ hrs reaction time. ${ }^{1}$ Furthermore, plete after $2-1 / 2$ hrs with the reagent concentrations given. 
when phenol was substituted for anisole in the Hamilton system, it was hydroxylated as shown by the decrease in absorbance (measured as the 4-AAP coupled dye) with time; presumably, phenol was converted to one or more dihydroxybenzenes. With the quantitative measurement employed here (oxidative coupling with 4-aminoantipyrine, which gives no interfering color with catechol), only the monohydroxy compound appears to be detected.

Figure 1 shows the time course for the hydroxylation of anisole with the Hamilton reagent as a function of the initial ratio of peroxide to aromatic. The absorbance is that of the 4-AAP coupled product. At ratios greater than 2 (filled circles), production and then loss of the monohydroxy compound occurs; at a ratio of 2.3 , stable absorbance values (and maximum yield) are obtained. ${ }^{2}$ When this ratio is reduced to values less than 2 (Figure 2, filled circles), stable absorbance values are again obtained, but the yield is dramatically reduced.

The effect of the initial ratio of peroxide to anisole on yield is further illustrated in Figure 3, which compares a concentration-response curve for the hydroxylation of anisole at constant initial ratio (where $\left[\mathrm{H}_{2} \mathrm{O}_{2}\right]_{0}$ is varied as [anisole] $]_{0}$ is varied) with one in which the ratio is not held constant (i.e., $\left[\mathrm{H}_{2} \mathrm{O}_{2}\right]_{0}$ is constant, but [anisole] 0 is varied). For the former, a straight line results, but in a

2 This absorbance corresponds to a maximum yield of about $40 \%$. 
Figure 1: Change of absorbance with time (4-AAP finish) for hydroxylation of anisole at $25^{\circ}$ in the absence of oxygen at several initial peroxide/ anisole ratios: $5.26 \times 10^{-4} \underline{M}$ anisole, $4.26 \times 10^{-5} \underline{M} \mathrm{Fe}(\mathrm{III}), 1.17 \times 10^{-4} \underline{\mathrm{M}}$ catechol, pH 3.9

O. $1.23 \times 10^{-3} \underline{\mathrm{M}} \mathrm{H}_{2} \mathrm{O}_{2}$ initial ratio, 2.3

Q. $2.45 \times 10^{-3} \underline{\mathrm{H}_{2}} \mathrm{O}_{2}{ }^{\prime}$ initial ratio, 4.7

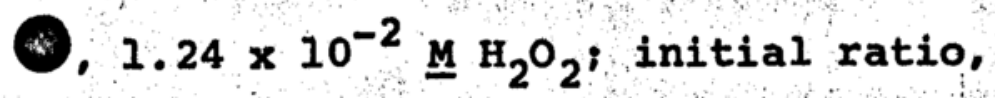
23.6

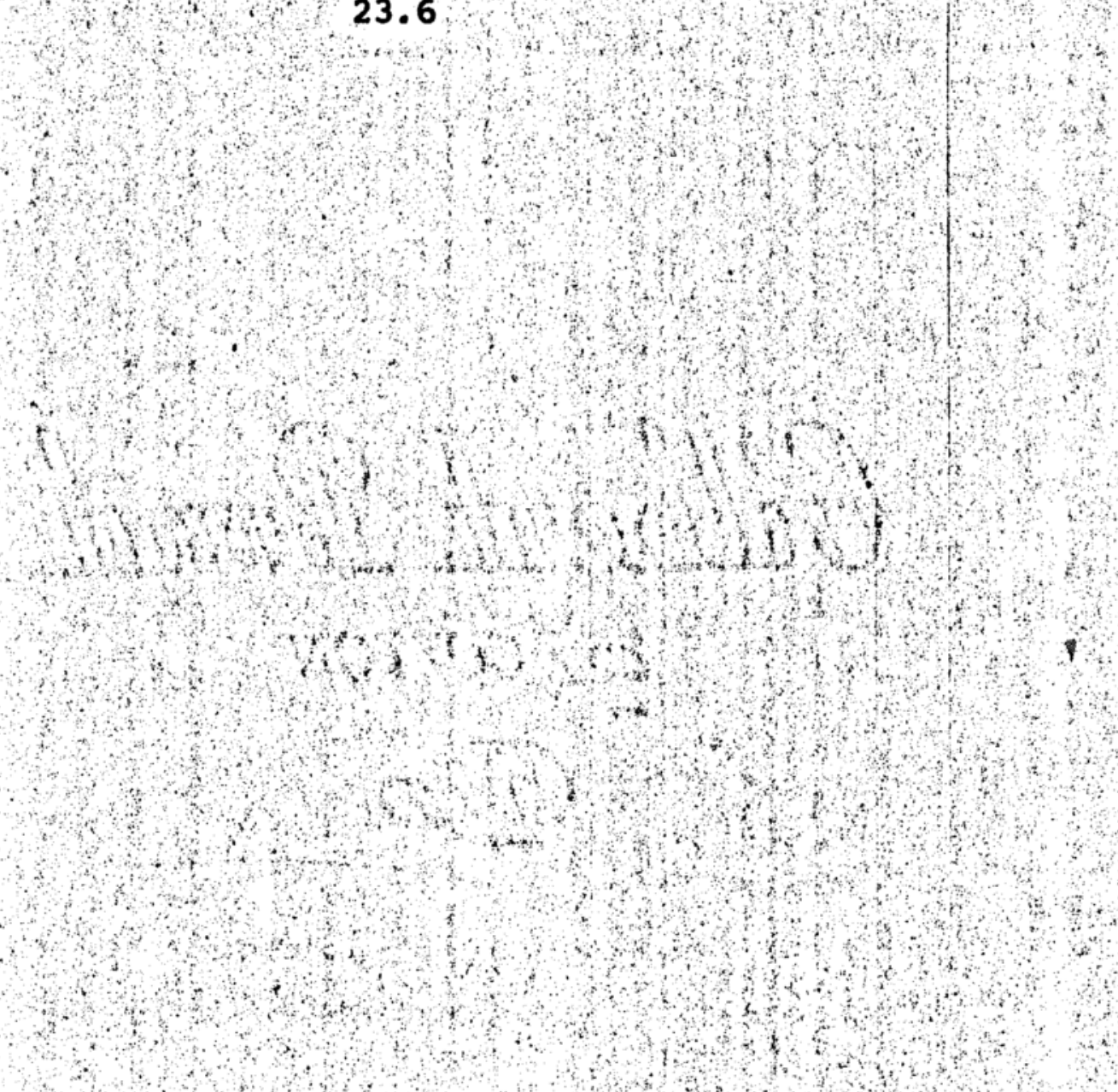




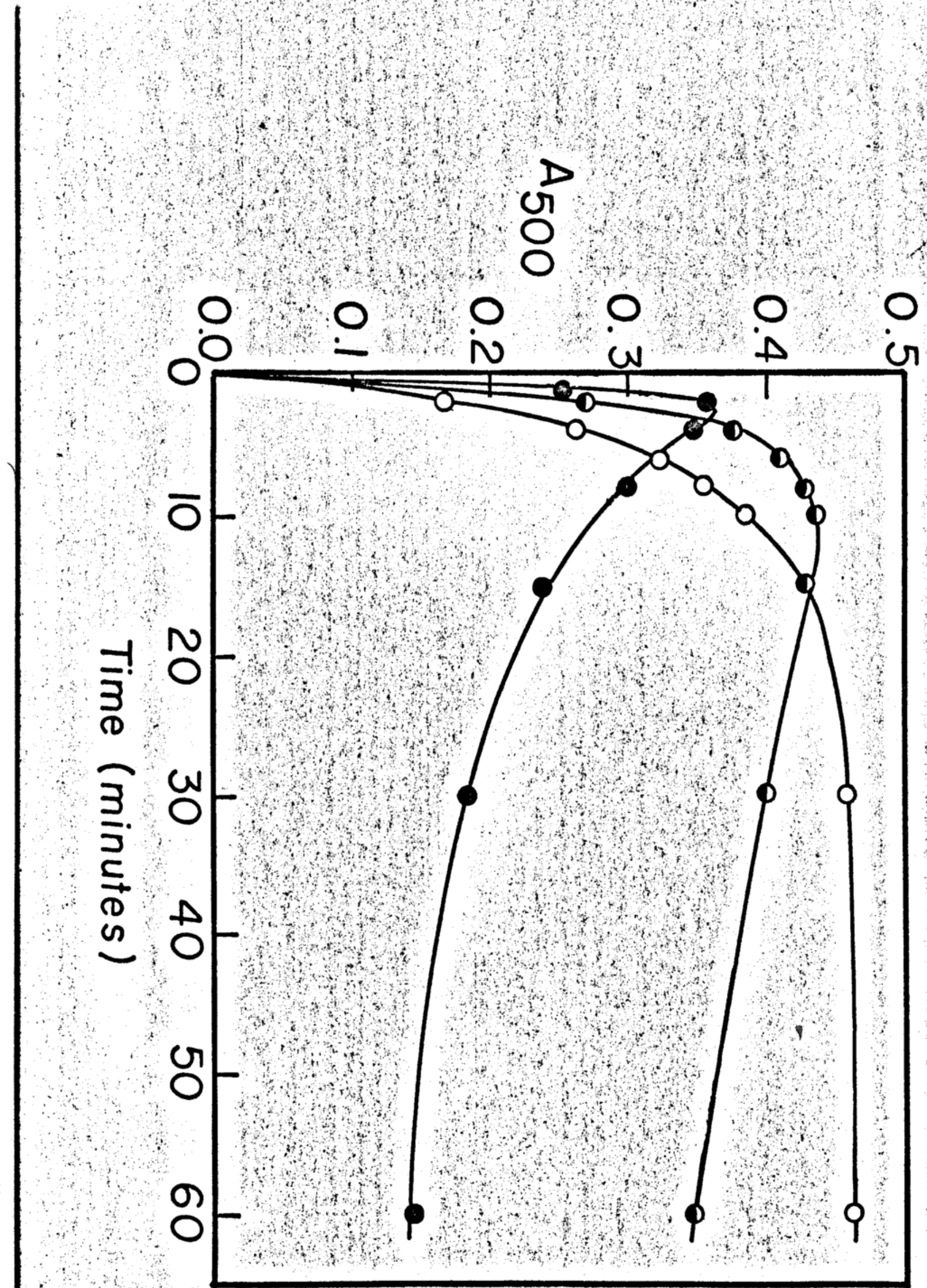


Figure 2: Change of absorbance with time (4-AAP finish) for hydroxylation of anisole at $25^{\circ}$ in the absence of oxygen: $5.26 \times 10^{-4} \underline{M}$ anisole. $4.26 \times 10^{-5} \underline{\mathrm{M} e}$ (III), $1.17 \times 10^{-4} \underline{\mathrm{M}}$ catechol. pH 3.9

O. $1.23 \times 10^{-3} \mathrm{M} \mathrm{H}_{2} \mathrm{O}_{2}$ initial ratio, 2.3

O. $5.93 \times 10^{-3} \mathrm{M} \mathrm{H}_{2} \mathrm{O}_{2}$ initial ratio, 1.1

, $2.97 \times 10^{-4} \mathrm{M}_{2} \mathrm{O}_{2}$ initial ratio, 0.6 


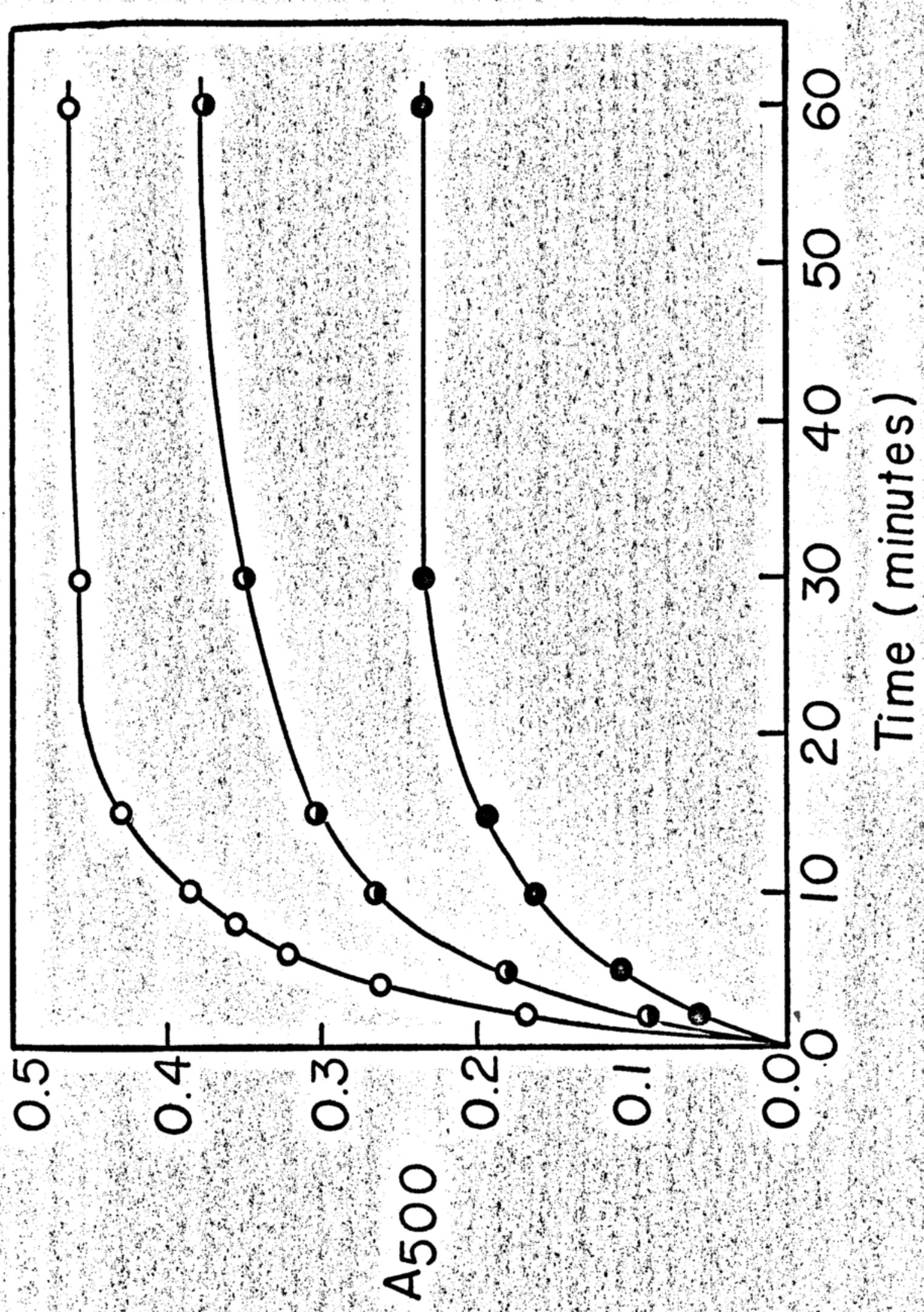




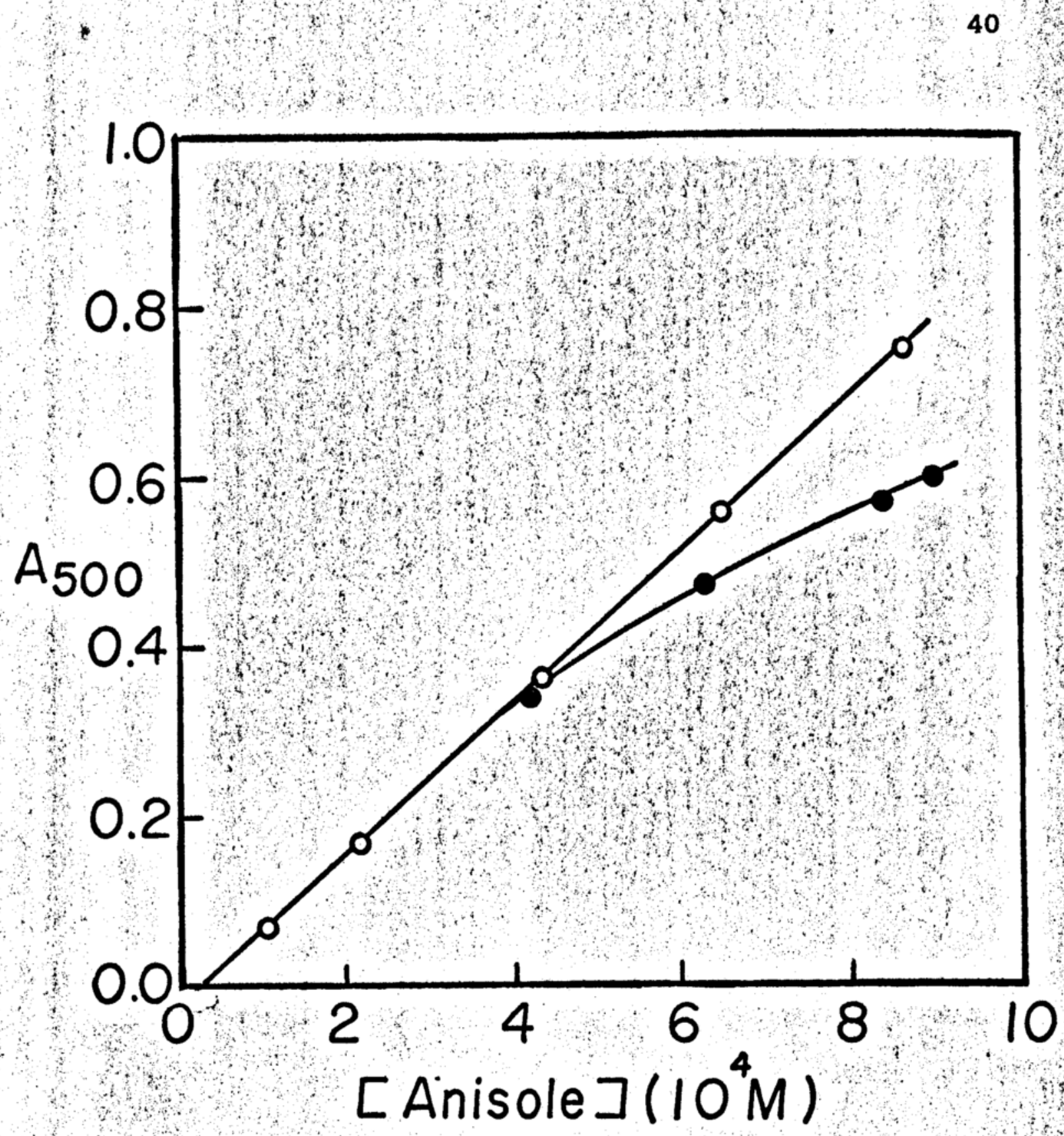

Figure 3: Concentration-response curves for hydroxylation of anisole in de-aerated water and a nitrogen atmosphere, $4.26 \times 10^{-5} \mathrm{M} F\left(\right.$ III), $1.17 \times 10^{-4} \mathrm{M}$ a) catechol, pH $3.9,1 \mathrm{hr}$ reaction time, $25^{\circ}, 4-\mathrm{AAP}$ finish.

$\mathrm{O}\left[\mathrm{H}_{2} \mathrm{O}_{2}\right] \mathrm{O}$ [Anisole $]_{0}=2.1$

- $\left[\mathrm{H}_{2} \mathrm{O}_{2}\right]_{\mathrm{O}}=8.21 \times 10^{-4} \mathrm{M}$ 
practical analytical situation this ratio cannot be held constant without prior knowledge of substrate concentration. The outcome is the result shown in Figures 1 and 2 and $a$ curved absorbance-concentration plot (Figure 3, filled circles). Though a curved absorbance-concentration plot does not prevent analytical use, there is no doubt that most analysts prefer to work with linear plots.

Figures 1,2 and 3 report data for the hydroxylation of anisole in de-aerated water under a nitrogen atmosphere. ${ }^{3}$ In the presence of oxygen, measurable, though small, differences in yield of monohydroxylated product were noted, but the time course for hydroxylation, Figure 4, revealing the production and then loss of monohydroxy product, is unchanged in general appearance.

2. Dependence of Yield on Catechol and Fe (III) Concentrations.

Hamilton $(17,18)$ observed a maximum rate for the loss of peroxide at a catechol concentration of $2.5 \times 10^{-4}$ M. His initial reactant concentrations were: $\mathrm{Fe}$ (III) $3.94 \times 10^{-5} \mathrm{M}$; anisole, $0.01 \mathrm{M} ; \mathrm{H}_{2} \mathrm{O}_{2}, 1.75 \times 10^{-3} \mathrm{M} ; \mathrm{pH} 4.3$ in $0.005 \mathrm{M}$ acetate buffer. Based upon these data, the effect of catechol on the yield of monohydroxy product for the hydroxylation of

3

Water was de-aerated by bubbling nitrogen gas through water that had been boiled and allowed to cool to room temperature. A nitrogen atmosphere was maintained using balloons filled with nitrogen gas placed over burettes containing the reaction mixture. 


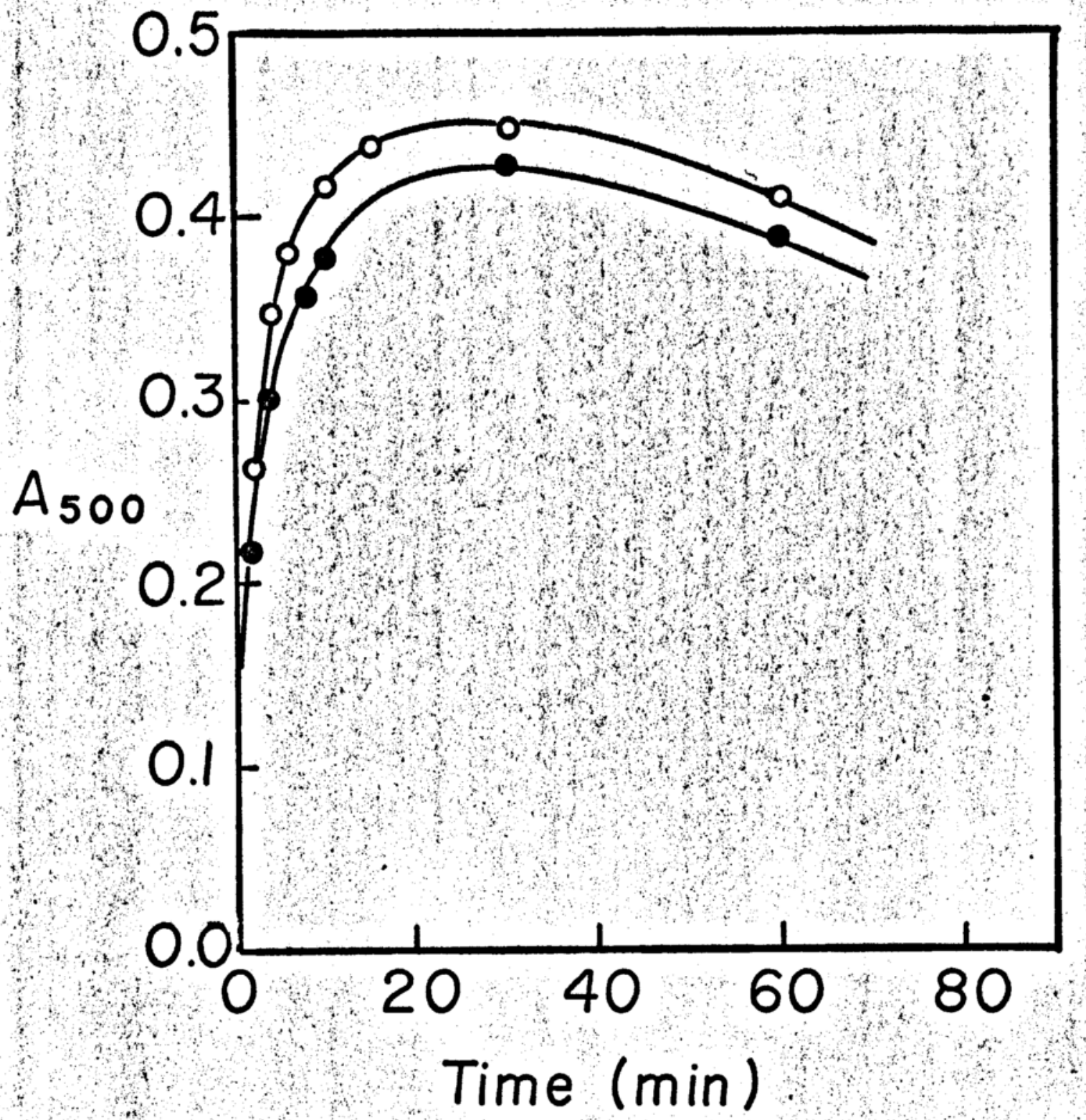

Figure 4: Change of absorbance (4-AAP finish) with time for hydroxylation of anisole at $25^{\circ}$ in presence and absence of oxygen; $4.26 \times 10^{-5} \underline{\mathrm{M} e}$ (III), $1.17 \mathrm{x}$ $10^{-4} \mathrm{M}$ catechol, $2.55 \times 10^{-3} \mathrm{M} \mathrm{H}_{2} \mathrm{O}_{2}, 5.39 \times 10^{-4} \mathrm{M}$ anisole, $\mathrm{pH} 3.9$.

$0 . \mathrm{O}_{2}$ absent

* $\mathrm{O}_{2}$ present 
anisole was investigated. Since the yield had been shown to be a function of the initial ratio of peroxide to anisole, this ratio was held constant at its maximum yield value of 2 (cf. Figures 1 and 2).

Table VI, summarizing the data, shows that a maximum yield is produced at about $1.2 \times 10^{-4}$ M catechol. To determine whether the absorption maximum was related to the ratio [Fe(III)]/[catechol], catechol concentration was kept constant at its optimum $\left(1.2 \times 10^{-4}\right.$ M) while Fe(III) was varied. The data, graphically depicted in Figure 5, establish that the ratio of catalysts contributes (however little), to the overall yield. Consequently, the effect of catechol concentration on yield was reinvestigated, but at a constant [Fe(III)]/ [catechol] ratio of 0.5 (which gives the maximum absorbance). The data, appearing in Table VII, demonstrate that an optimum catechol concentration does occur at a value of about $1.2 \times 10^{-4} \underline{\mathrm{M}}$.

\section{Dependence of Yield on $\mathrm{pH}$.}

Hamilton (18) indicated that the rate of loss of peroxide for the hydroxylation of anisole was independent of the $\mathrm{pH}$ from 3.5 to 4.2 . Table VIII illustrates that yield is essentially independent of the $\mathrm{pH}$ from 3.5 to 4.2 . The data , are depicted graphically in Figure 6.

\section{4. "Aging" of the Hamilton System.}

Catechol, itself an aromatic, should be hydroxylated, thereby altering its catalytic effectiveness as well as 


\section{Table VI}

Effect of [Catechol] ${ }_{0}$ on Yield for the Anerobic Hydroxylation of Anisole at $25^{\circ}$.

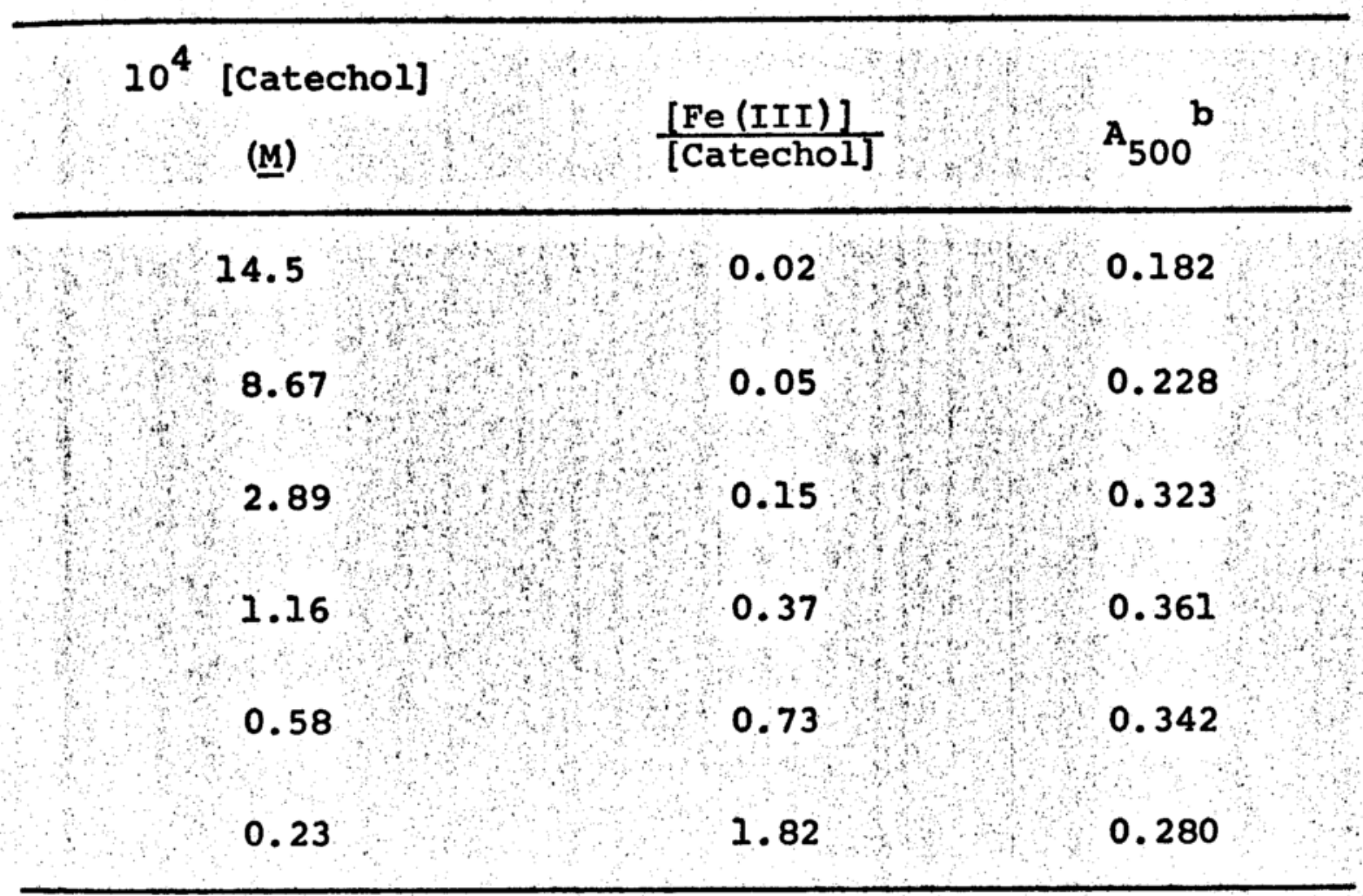

a $4.22 \times 10^{-4} \underline{M}$ anisole; $4.26 \times 10^{-5} \underline{M ~ F e}\left(\right.$ III) $; 8.01 \times 10^{-4} \underline{M}$ $\mathrm{H}_{2} \mathrm{O}_{2} ; \mathrm{pH} 3.9$; reaction time $60 \mathrm{~min}$.

b Absorbance values corrected for blank, from 4-AAP finish. 


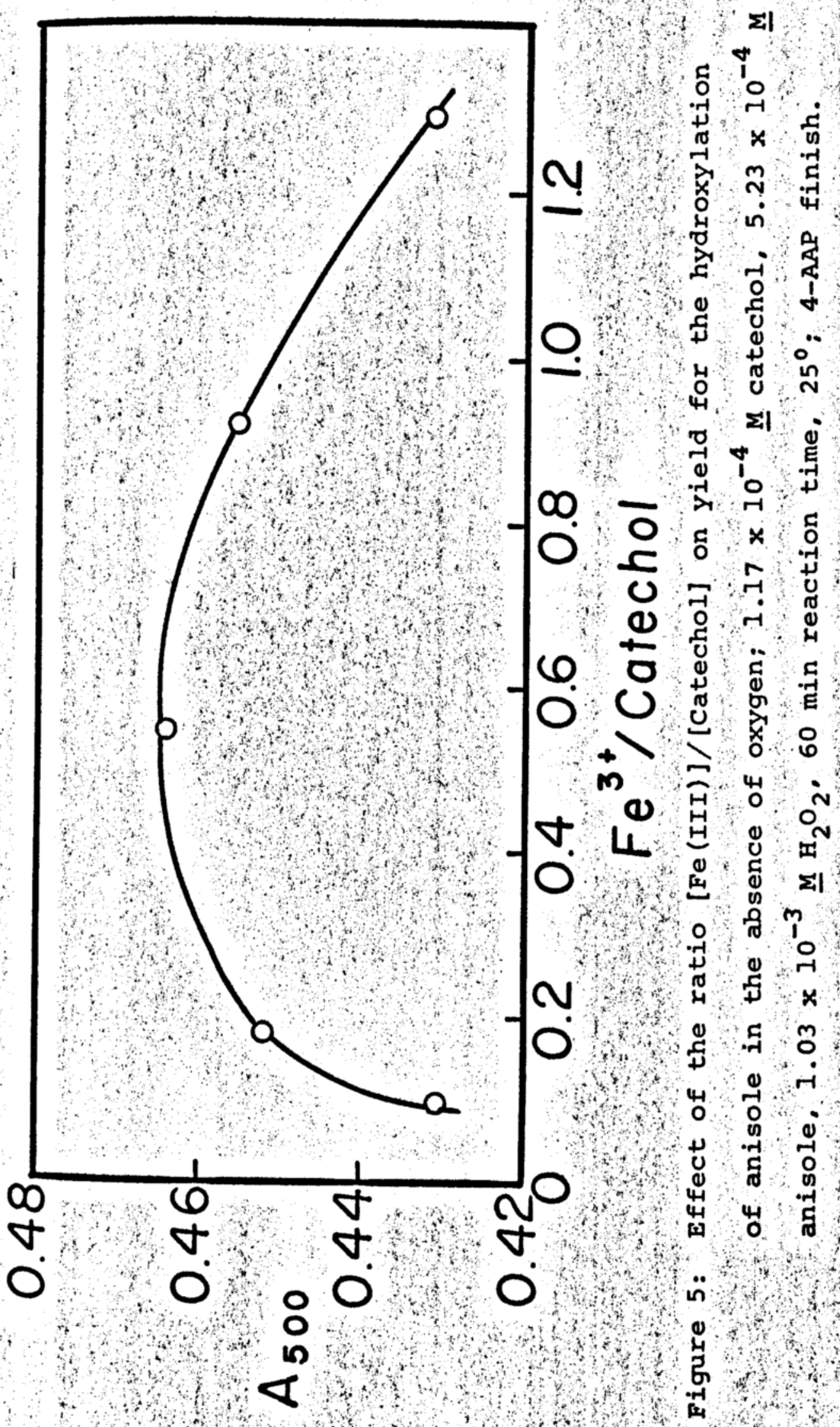


Table VII

Effect of [Catechol] $]_{0}$ on Yield at Constant [Fe(III)]/[Catechol], for the Anerobic Hydroxylation of Anisole at $25^{\circ}$. a

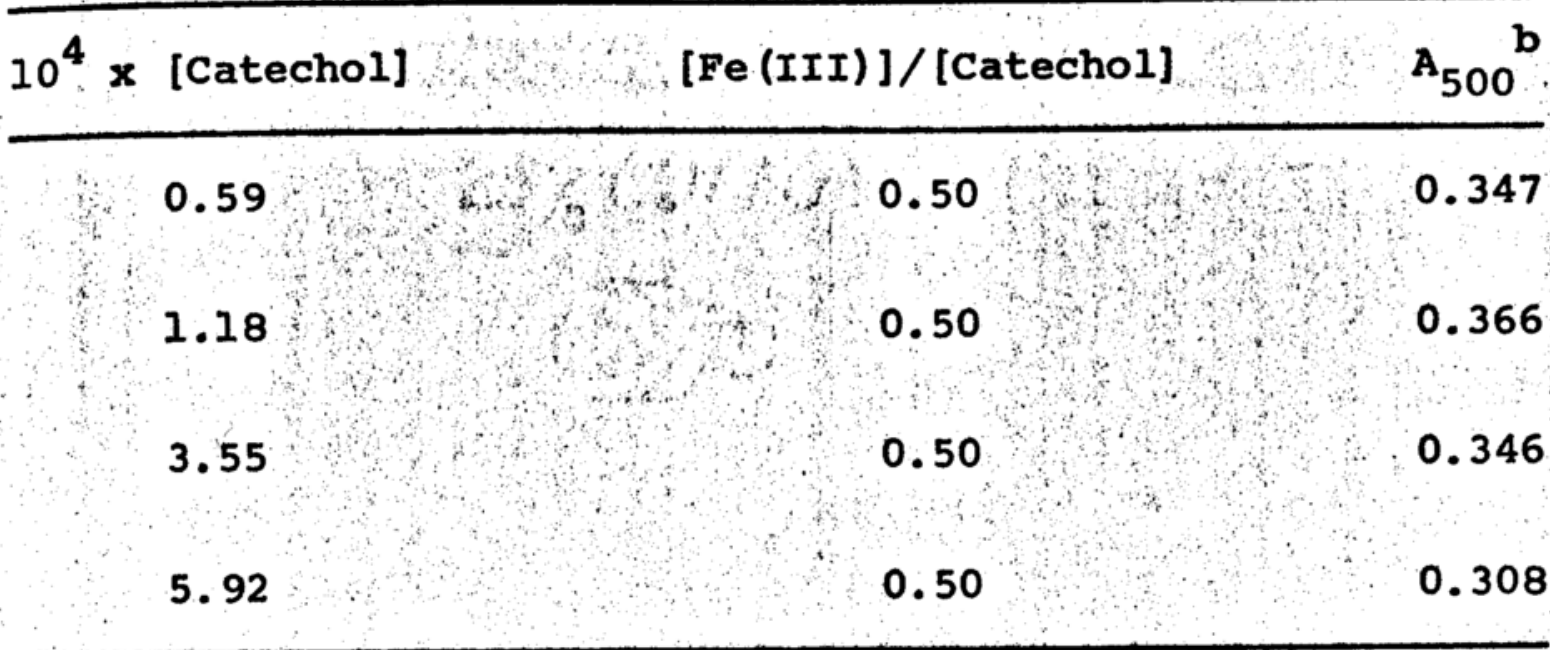

a $4.26 \times 10^{-4} \underline{M}$ anisole; $8.65 \times 10^{-4} \underline{M} \mathrm{H}_{2} \mathrm{O}_{2} ; \mathrm{pH} 3.9 ;$ reaction time $60 \mathrm{~min}$.

b Absorbance values corrected for blank, from 4-AAP finish. 
Table VIII

Dependence of Yield on pH for the Hydroxylation of Anisole at $25^{\circ}$ in the Absence of Oxygen.

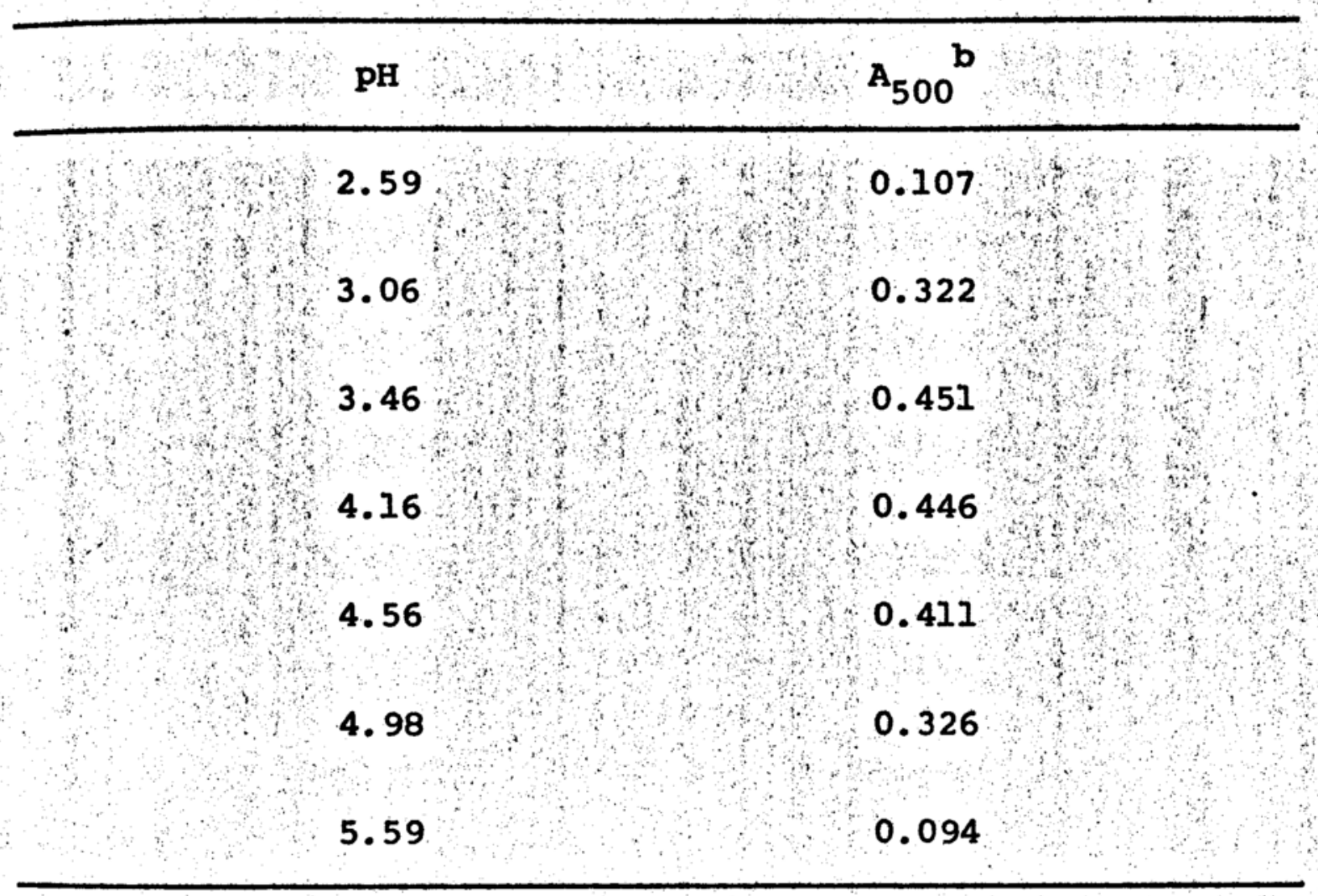

a $5.90 \times 10^{-5} \underline{M} \mathrm{Fe}(\mathrm{III}) ; 1.18 \times 10^{-4} \underline{M}$ catechol; $1.08 \times 10^{-3} \underline{M}$ $\mathrm{H}_{2} \mathrm{O}_{2} ; 5.33 \times 10^{-4} \underline{M}$ anisole; reaction time $60 \mathrm{~min}$.

b Absorbance values, corrected for blank, from 4-AAP finish. 


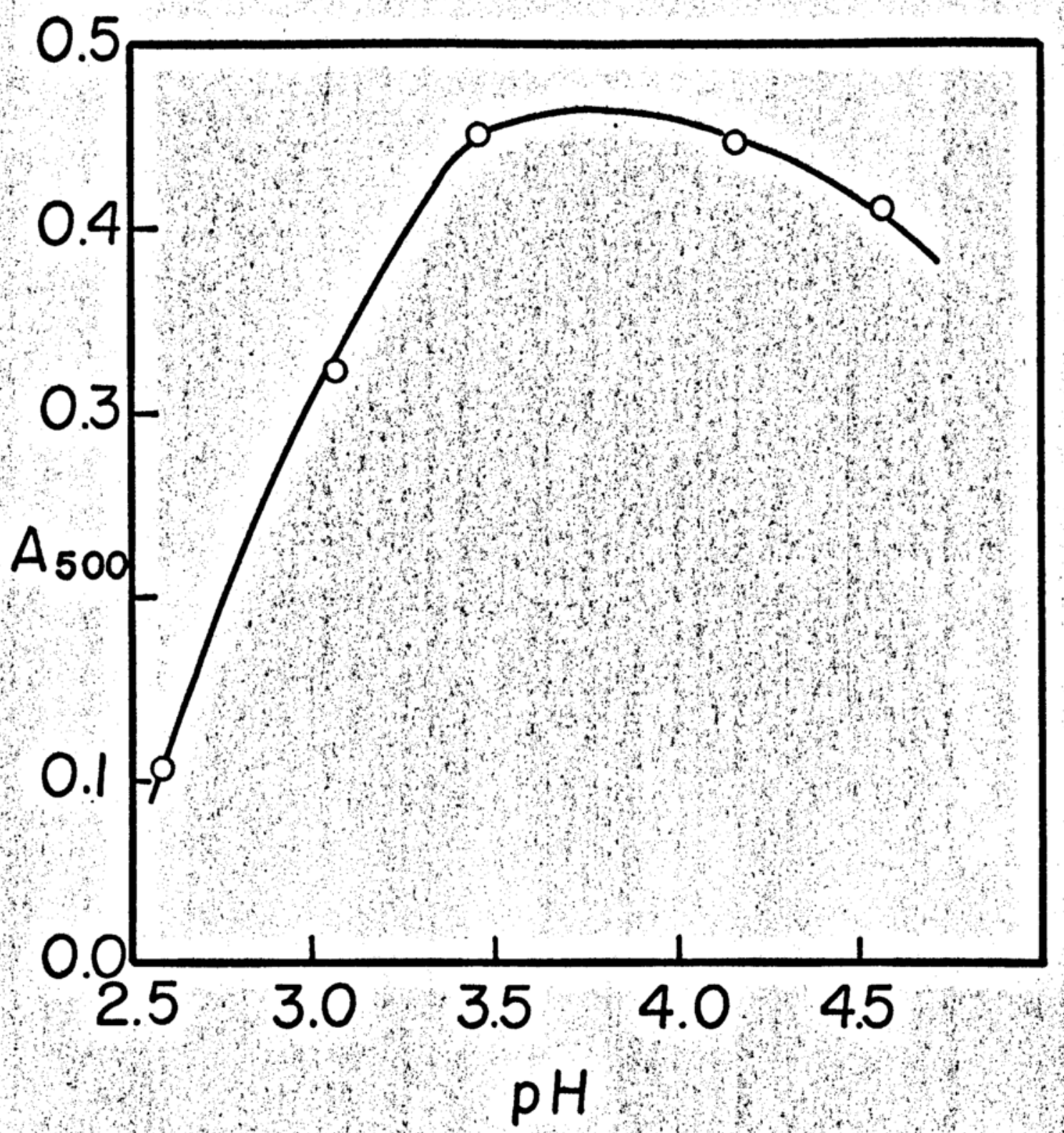

Figure 6: Dependence on pH of yield. Data from Table VIII. 
depleting peroxide. This "aging" phenomenon is dramatically illustrated in Table IX, which shows that the time of addition of substrate (anisole) to a pre-prepared Hamilton system affects the yield of monohydroxy compound. The longer the system is allowed to age (in the absence of anisole), the lower the yield. Furthermore, the data indicate that the catalytic effectiveness of catechol is markedly altered with aging because when additional catechol is added to an aged solution (additional $\mathrm{H}_{2} \mathrm{O}_{2}$ was also added), the yield returns to the expected value. In the absence of added enediol however, no further increase in yield is noted.

B. Hydroxylation with a Cyclodextrin-Modified Hydroxylating System

1. Stabilizing Effect of Cyclodextrins.

Figure 1 shows that under analytical conditions, where the hydrogen peroxide in the Hamilton system is in excess of the aromatic sample compound, hydroxylation continues beyond the introduction of the first hydroxy group. This destruction of the monohydroxy product by rapid subsequent hydroxylation therefore renders the Hamilton system ineffective as an analytical reagent.

One approach in circumventing the instability of the initial product of hydroxylation that has been tried (with a successful method resulting), is to stabilize it by the addition of a cyclodextrin. (The cyclodextrin effect will be 
Table IX

Effect of Aging on Yield for the Anerobic Hydroxylation of Anisole at $25^{\circ}$.

Age of Hamilton system

before addition of anisole $\quad A_{500}$ (4-AAP finish)

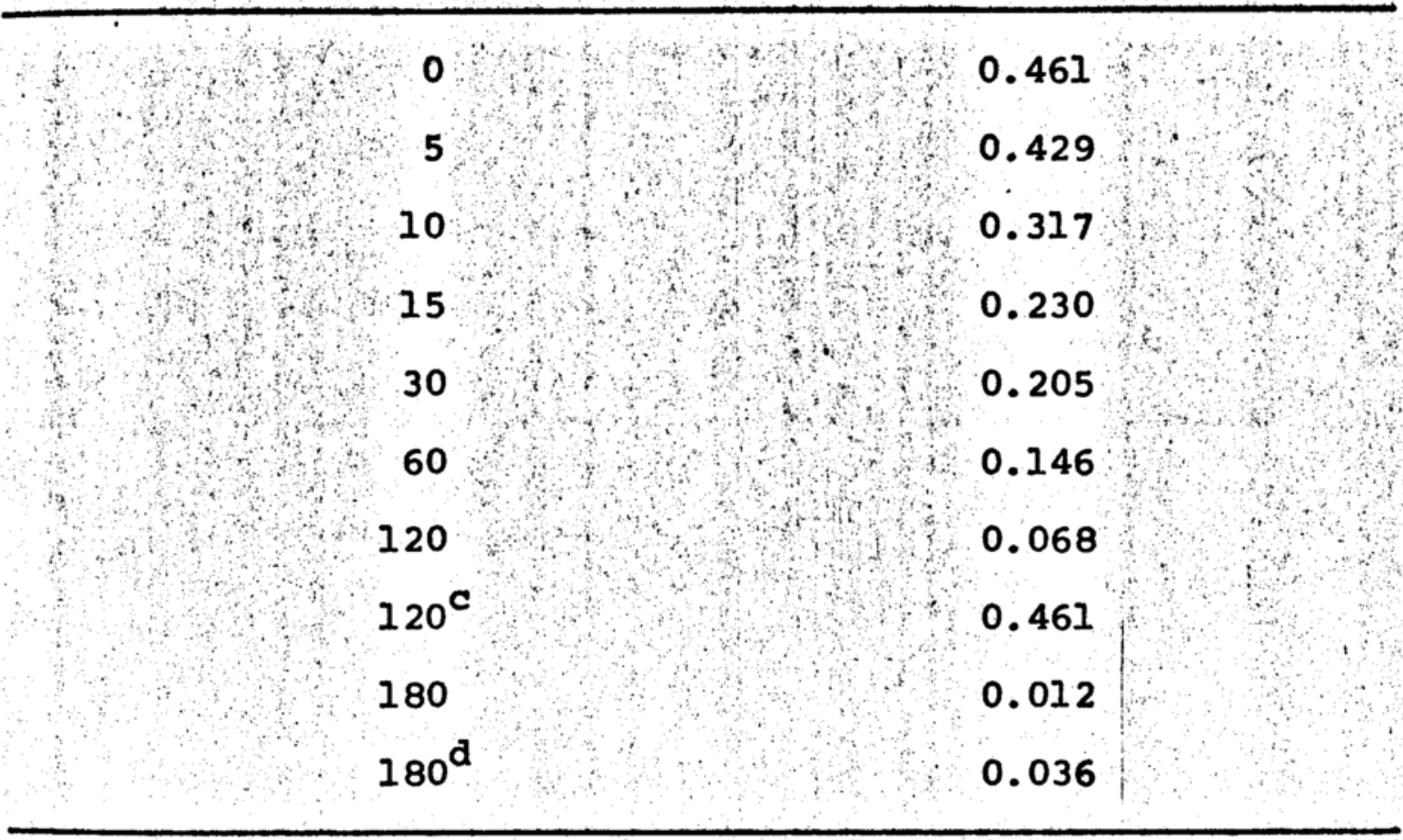

a $5.36 \times 10^{-4} \underline{M}$ anisole; $5.90 \times 10^{-5} \underline{\mathrm{M}} \mathrm{Fe}(\mathrm{III}) ; 1.17 \times 10^{-4} \underline{\mathrm{M}}$ catechol; $1.06 \times 10^{-3} \underline{\mathrm{M}} \mathrm{H}_{2} \mathrm{O}_{2} ; \mathrm{pH} 3.9 ; 60$ min reaction time.

b Corrected for blank.

C Additional catechol $\left(1.17 \times 10^{-4} \underline{\mathrm{M}}\right)$ and $\mathrm{H}_{2} \mathrm{O}_{2}\left(1.06 \times 10^{-3} \mathrm{M}\right)$ added at this time.

Additional $\mathrm{H}_{2} \mathrm{O}_{2}\left(1.06 \times 10^{-3} \mathrm{M}\right)$ added at this time. 
discussed in the Discussion Section.) Figure 7 , showing the effect of cyclodextrin concentration on the time course for hydroxylation of anisole 4 . gives the results of an initial experiment that encouraged further investigation, since it revealed that moderate stabilization could be achieved at high ratios of peroxide to sample. The yield is dependent upon the initial ratio of peroxide to sample as well as the ratio of cyclodextrin to sample. In this experiment, the former ratio was 33 whereas the latter was varied at 0.156 , 1.56 and 15.6. At high cyclodextrin concentrations ([Cyclodextrin]/[Anisole] -15.6 ), the yield is severely depressed although stability is achieved (filled circles); too little cyclodextrin, on the other hand, produces no stabilizing effect (open circles, ratio $=0.156$ ). At a ratio of cyclodextrin to anisole of 1.56 , however, both reasonable stability and reasonable yield ensue (half-filled circles).

The effect of the initial ratio of peroxide to anisole on yield at fixed cyclodextrin to anisole ratio is depicted in Figure 8. It is apparent that too much (filled circles) or too little peroxide (half-filled circles) depresses the yield (presumably because of sequential hydroxylation and insufficient reaction, respectively).

4 These and all subsequent studies were run in an air environment. Although yields were slightly decreased when oxygen was present (Figure 4), the simplicity of running reaction under "aerobic" conditions more than offsets the modest loss in yield. 
Figure 7: Change of absorbance (4-AAP finish) with time for hydroxylation of anisole at $25^{\circ}$ in aqueous solution; $5.54 \times 10^{-4} \underline{M}$ anisole; $1.18 \times 10^{-4} \underline{M}$ catechol, $5.92 \times 10^{-5} \underline{\mathrm{MF}}$ (III) $1.83 \times 10^{-2} \underline{M}$ $\mathrm{H}_{2} \mathrm{O}_{2} ; \mathrm{pH} 3.8$

O. $8.48 \times 10^{-5} \underline{\text { M }}$-cyclodextrin

Q $8.48 \times 10^{-4} \mathrm{M} \beta$-cyclodextrin

* $8.48 \times 10^{-3}$ M $\beta$-cyclodextrin 


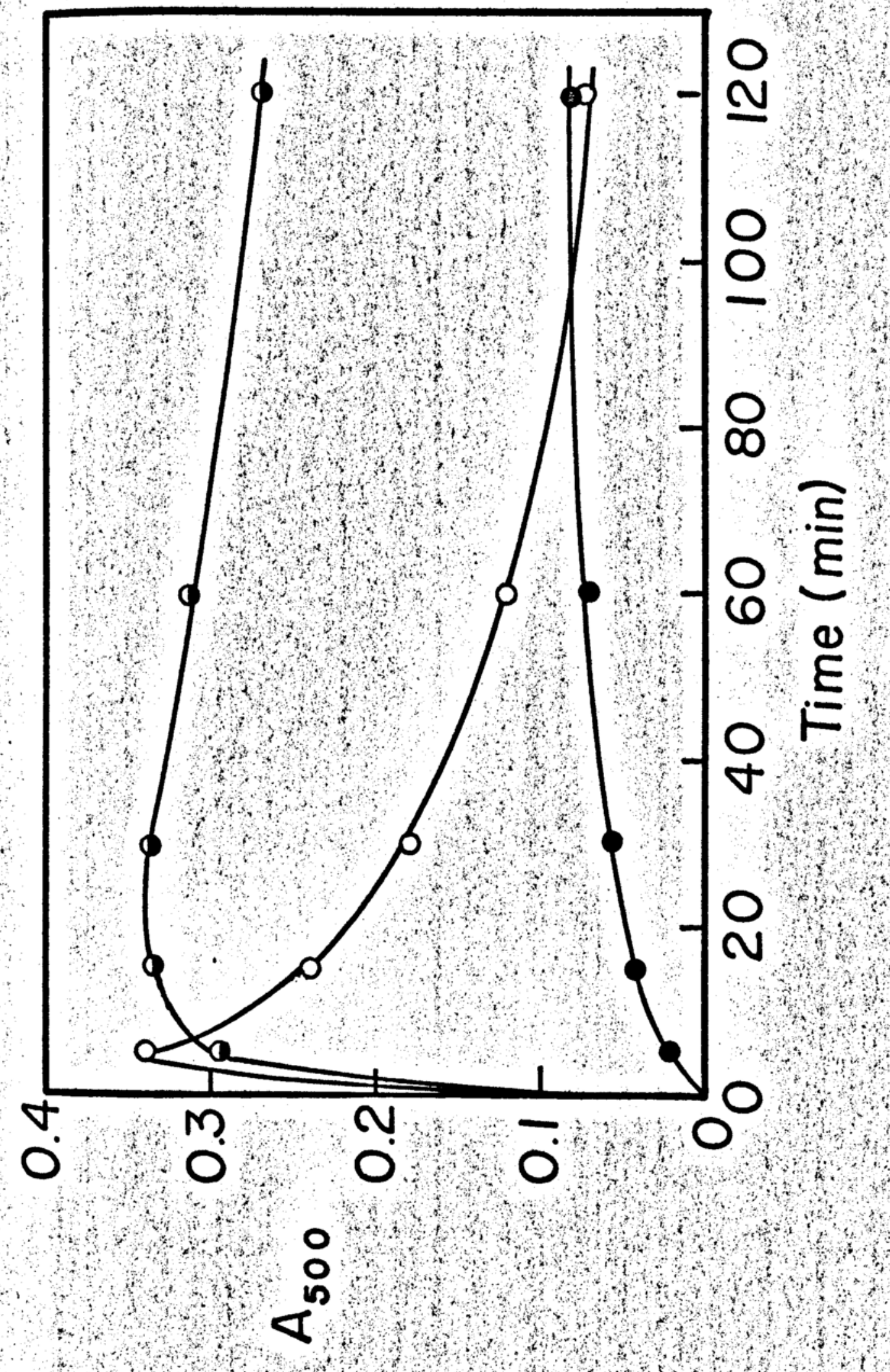


Figure 8: Effect of the ratio of peroxide to anisole on the time course for the cyclodextrinmediated hydroxylation of anisole in aqueous solution at $25^{\circ}, 6.39 \times 10^{-4} \mathrm{M}$ anisole, $1.17 \times 10^{-4} \underline{M}$ catechol, $5.94 \times 10^{-5} \underline{M} \mathrm{Fe}(\mathrm{III})$, $8.92 \times 10^{-4} \underline{M} \beta$-cyclodextrin, $\mathrm{pH} 3.8,4-\mathrm{AAP}$ finish.
O. $3.27 \times 10^{-3} \mathrm{M} \mathrm{H}_{2} \mathrm{O}_{2}$
○. $7.24 \times 10^{-2} \mathrm{M} \mathrm{H}_{2} \mathrm{O}_{2}$
(2) $7.24 \times 10^{-4} \underline{\mathrm{M}} \mathrm{H}_{2} \mathrm{O}_{2}$ 


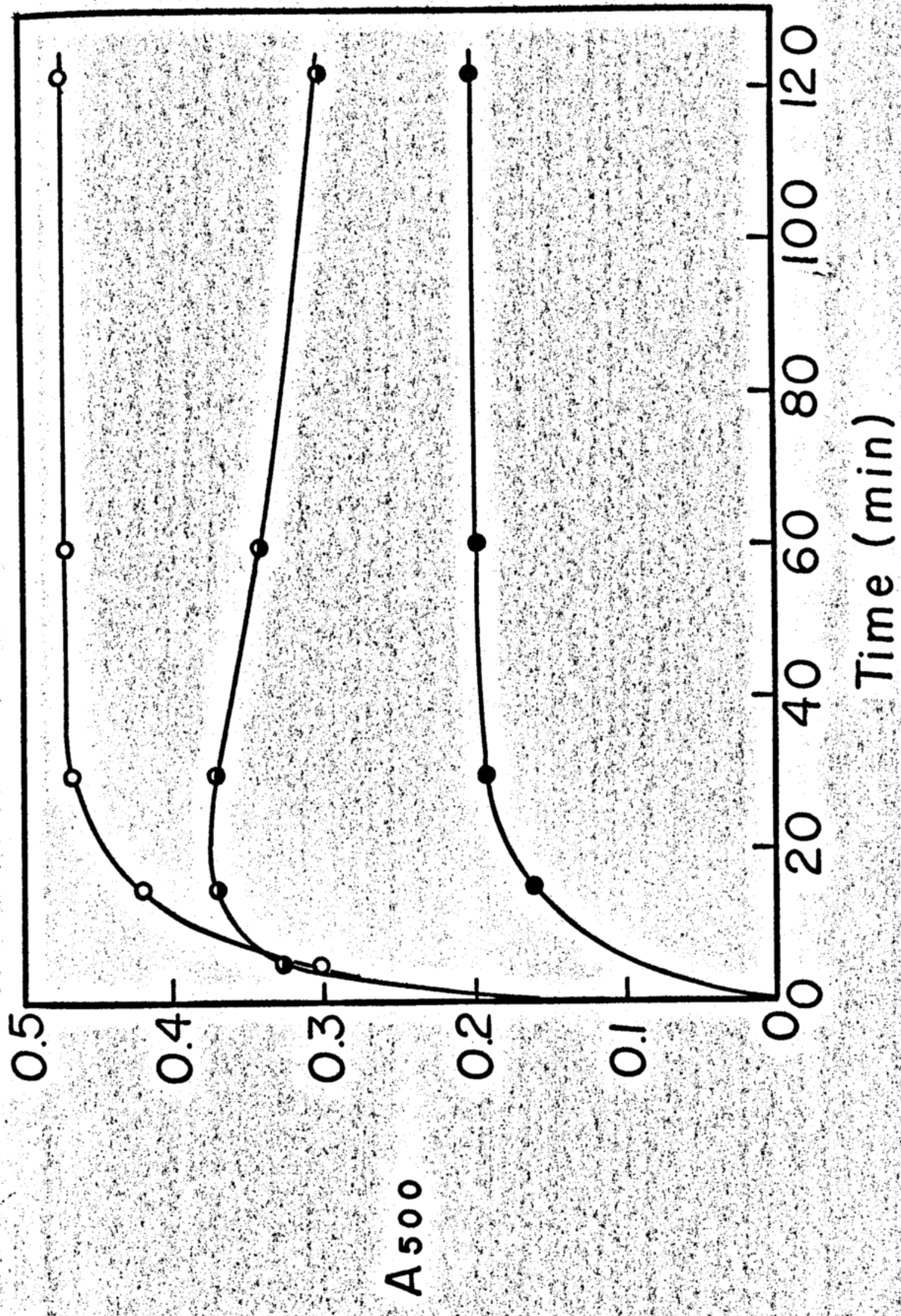


The data from Figures 7 and 8 suggest that, if sufficient cyclodextrin is incorporated into the reaction mixture, then a constant percentage yield of monohydroxy product, independent of the initial ratio of peroxide to substrate, will result. Provision of course, must be made for the acceptable range over which the ratio is allowed to vary (cf. Figure 8).

A concentration-response curve for the hydroxylation of anisole is depicted in Figure 9. A constant yield for the initial product of hydroxylation is reflected in the linearity of the graph. 5 The initial ratio of peroxide to anisole varied from about 6 to 35 while the ratio of cyclodextrin to anisole was in the range 1.6 to 10. It should be noted that as the peroxide-anisole ratio becomes smaller ${ }^{6}$, the need for the stabilizing effect of cyclodextrin diminishes because sequential hydroxylation is not as pronounced (cf. Figure 1). The choice of reaction time $(60 \mathrm{~min})$ was based upon the shape of the time course curve. Inspection of Figure 8 reveals that, in the presence of sufficient cyclodextrin and peroxide (open circles), the reaction with anisole apparently gives a limiting yield in $60 \mathrm{~min}$. Figure 10, showing the time course for hydroxylation of benzene in the presence and

5 The nature of the negative intercept will be discussed later.

6

The cyclodextrin-anisole ratio becomes smaller also. 


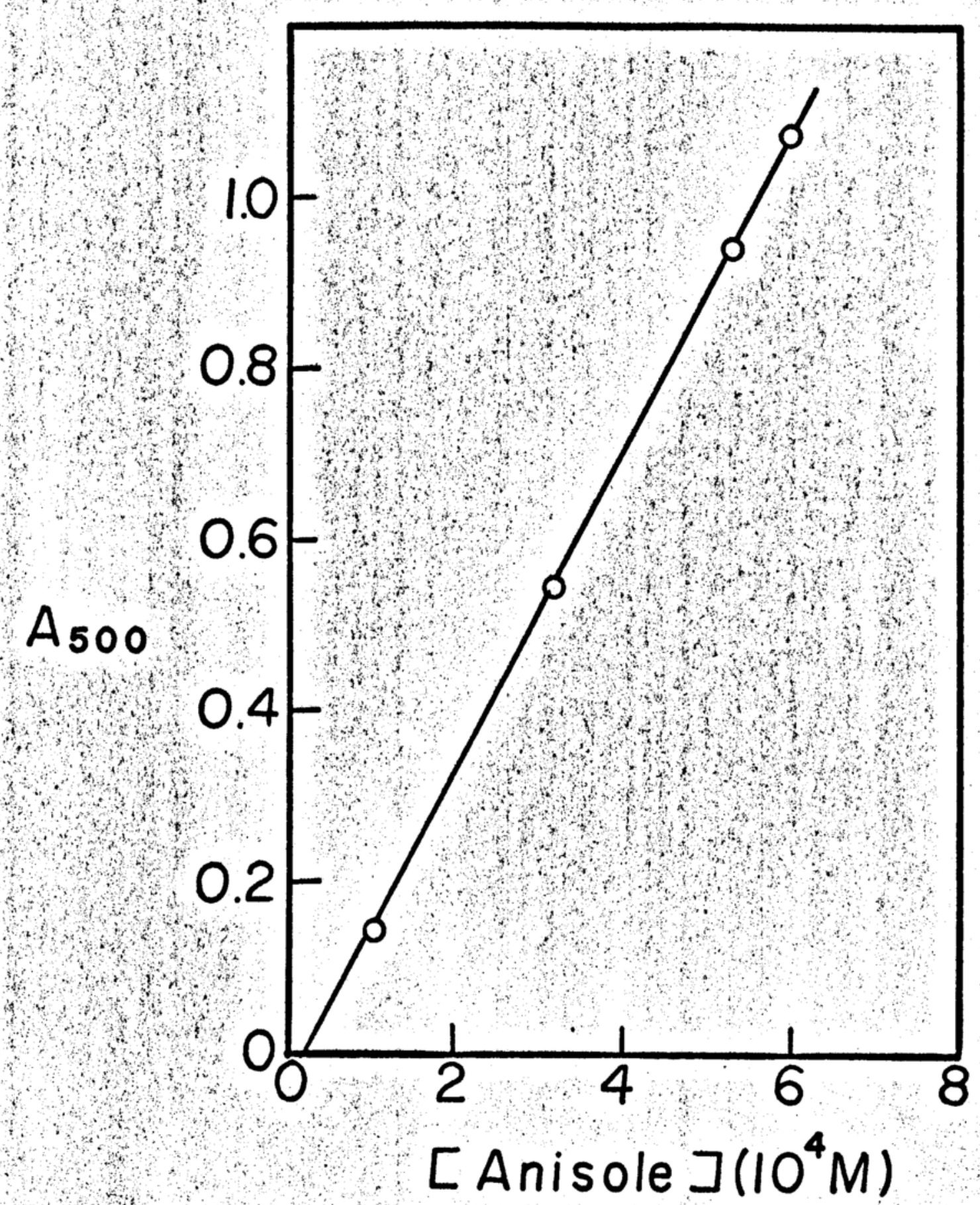

Figure 9: Concentration-response curve for the hydroxylation of anisole at $25^{\circ}, 1.17 \times 10^{-4} \underline{M}$ catechol, $5.94 \times$ $10^{-5} \mathrm{M} \mathrm{Fe}(\mathrm{III}), 3.53 \times 10^{-3} \mathrm{M} \mathrm{H} \mathrm{O}_{2}, 1.0 \times 10^{-3} \mathrm{M}$ cyclodextrin, $60 \mathrm{~min}$ reaction time, 4-AAP finish. 
Figure 10: Time course for hydroxylation of benzene in

the presence and absence of cyclodextrins

at $25^{\circ}, 5.38 \times 10^{-4} \underline{M}$ benzene, $1.17 \times 10^{-4} \underline{M}$

catechol, $5.94 \times 10^{-5} \underline{M F e}$ (III), $3.63 \times$

$10^{-3} \underline{\mathrm{M}} \mathrm{H}_{2} \mathrm{O}_{2}, \mathrm{pH} 3.7,4-\mathrm{AAP}$ finish.

; no cyclodextrin

( ) $\beta$-cyclodextrin, $1.13 \times 10^{-3} \mathrm{M}$

* $\alpha$-cyclodextrin, $1.26 \times 10^{-3} \mathrm{M}$ 


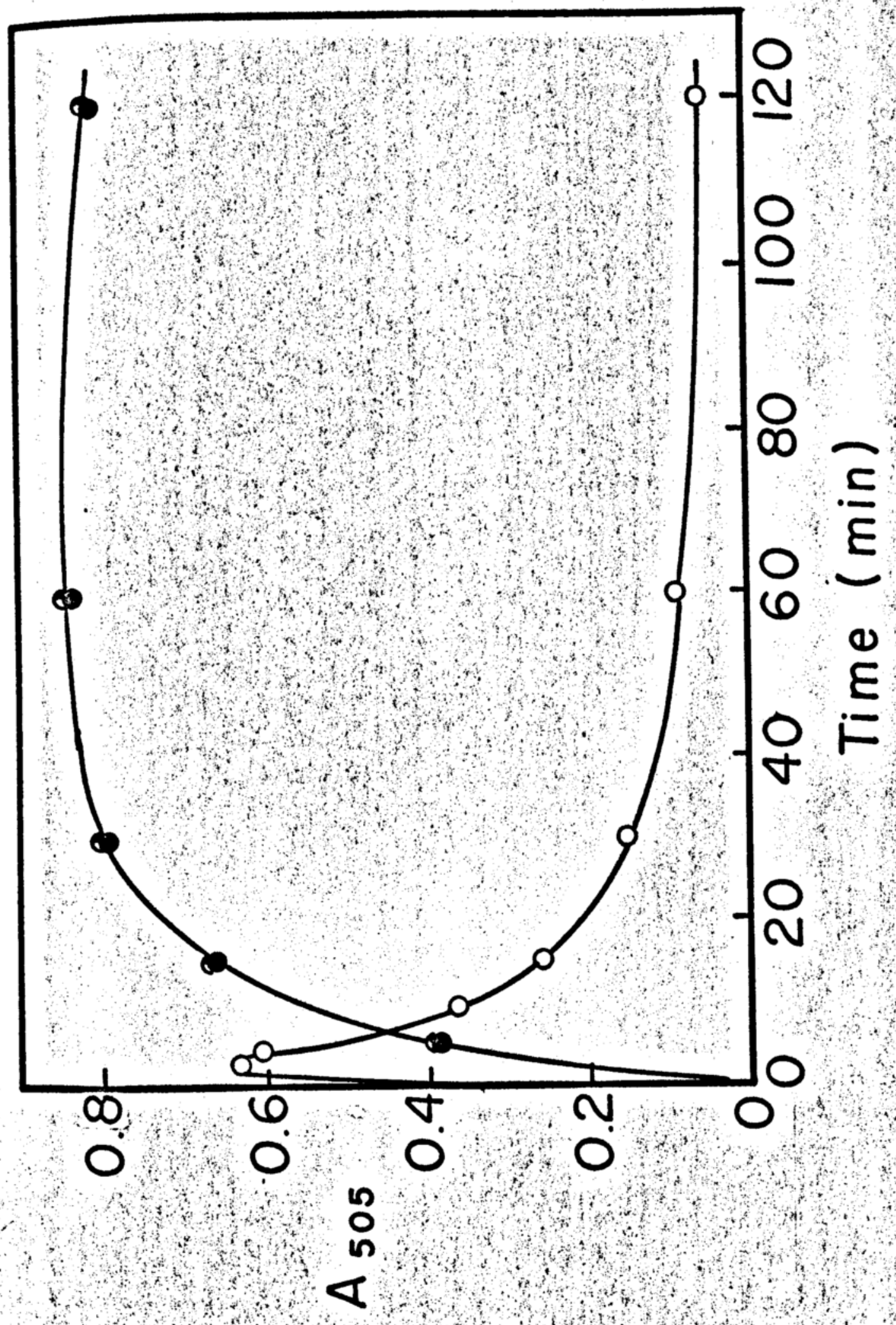


absence of cyclodextrins, illustrates that a 60 min reaction time is again appropriate. Figure 10 also reveals that $\alpha$ cyclodextrin and $\beta$-cyclodextrin show similar behavior in the hydroxylation system; both indeed stabilize the initial hydroxylation product.

Figure $I 1$ is a Beer's Law plot for the treatment of benzene with the cyclodextrin-modified Hamilton reagent. A linear curve results, indicating constant yields over the range of initial peroxide-benzene ratios 3.15 to 31.5 . The cyclodextrin to benzene ratio varied from 0.98 to 9.8 .

Figure 12, showing the time course of the treatment of nitrobenzene with the Hamilton system with and without cyclodextrins, reveals that a 60 minute reaction time is insufficient for complete hydroxylation. Consequently, samples were allowed to react for 2 hours prior to final analytical measurement. With this substrate, color development by the 4aminoantipyrine method was omitted and the nitrophenolate absorption was measured at pH 8.6. More will be said about this later. The stabilizing effects exhibited by $\alpha$ - and $\beta-$ cyclodextrin appear to be similar, consistent with previous observations.

2. Effect of $\mathrm{pH}$ and Buffer Concentration.

The dependence of $\mathrm{pH}$ on yield for the cyclodextrinmediated hydroxylation of acetanilid, depicted in Figure 13, is consistent with previous data for anisole hydroxylation in an unmodified Hamilton system (Figure 6). The yield is 
61

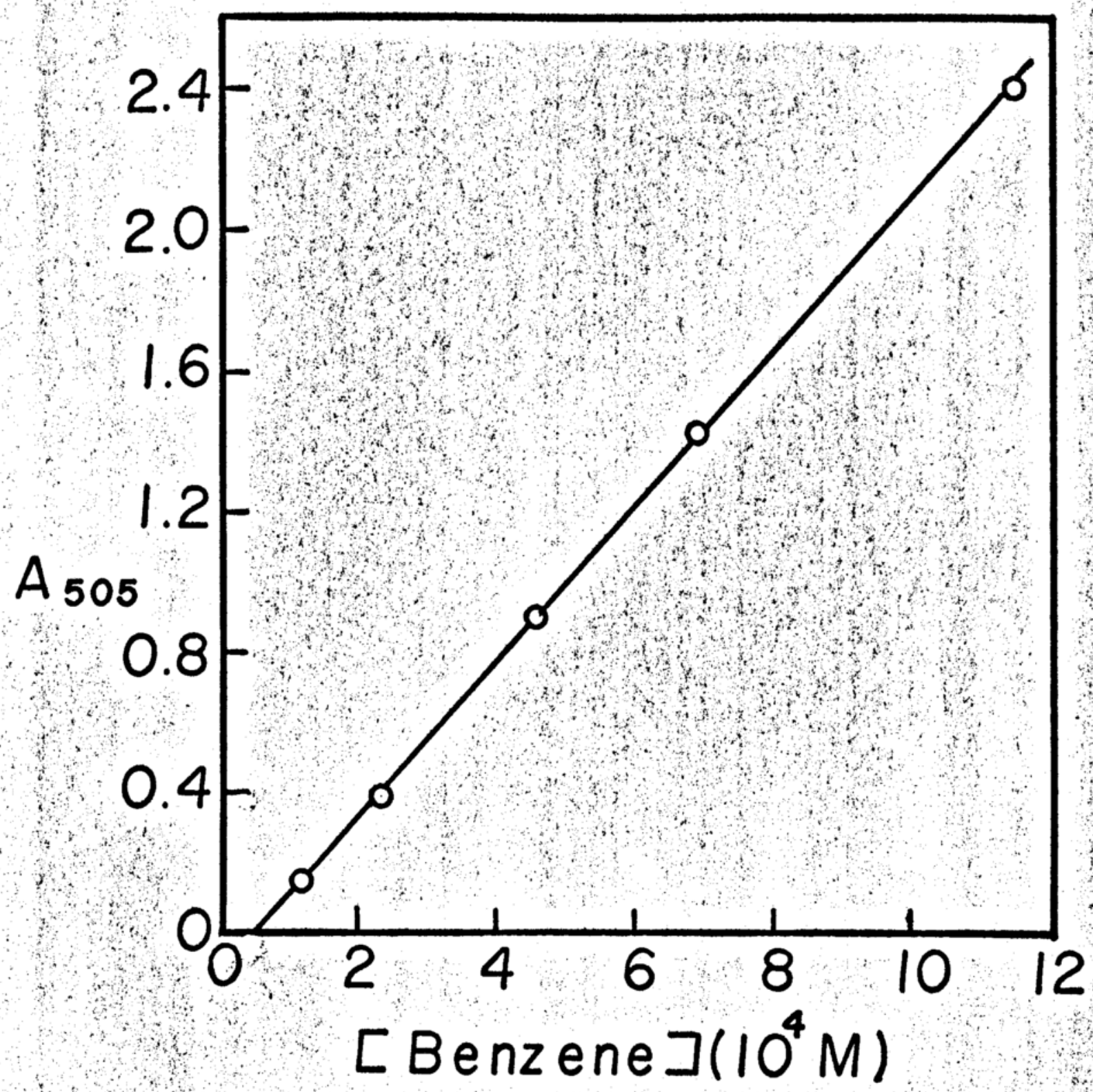

Figure 11, Concentration-response curve for the hydroxylation of benzene at $25^{\circ}, 1.18 \times 10^{-4}$ M catechol, $5.94 \times$ $10^{-5} \underline{\mathrm{M} e}(\mathrm{III}), 3.62 \times 10^{-3} \underline{\mathrm{M}} \mathrm{H}_{2} \mathrm{O}_{2}, 1.13 \times 10^{-3} \underline{\mathrm{M}}$ $\beta$-cyclodextrin, 60 min reaction time; 4-AAP finish. 


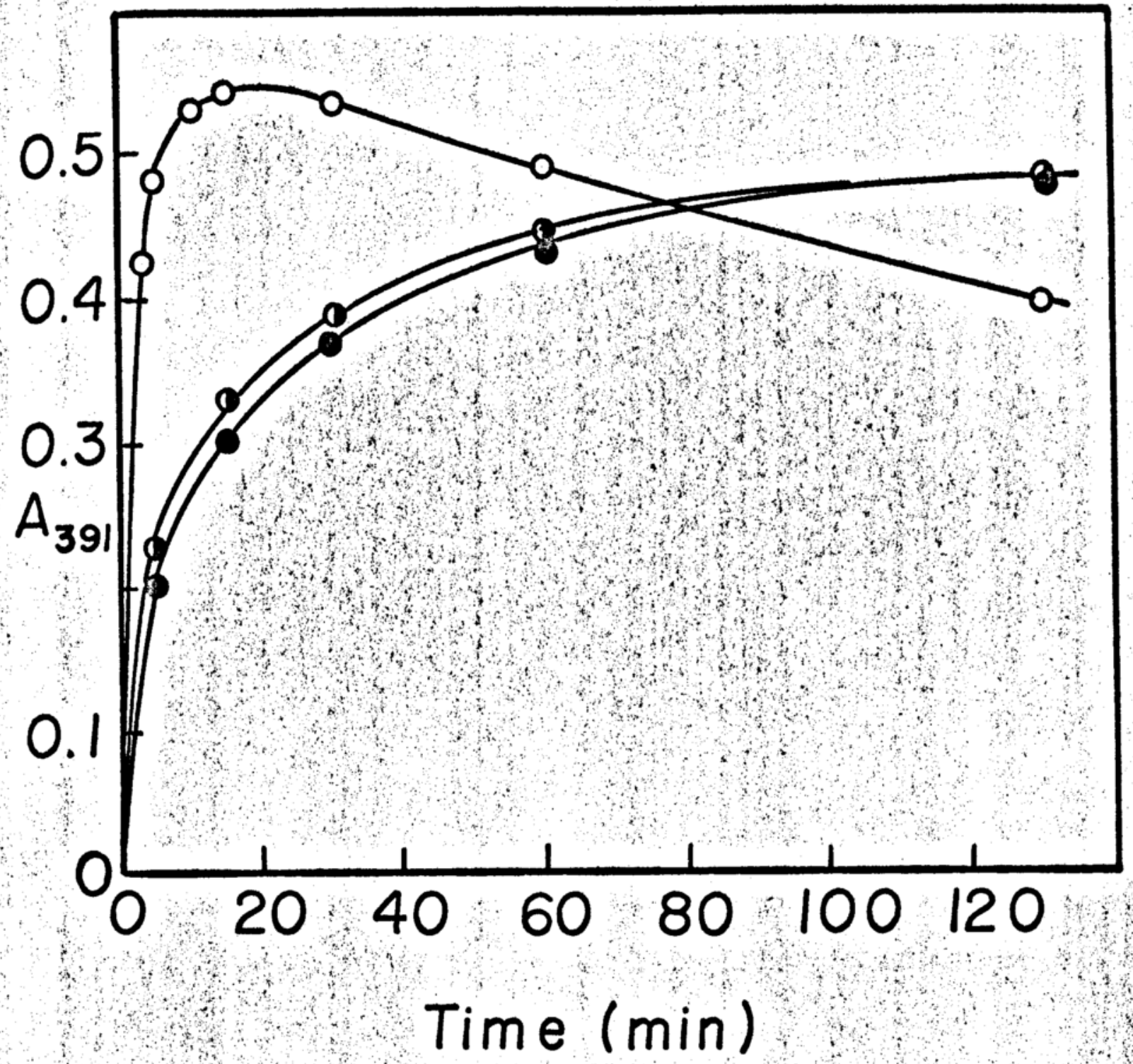

Figure 12, Change of absorbance with time for hydroxylation of nitrobenzene at $25^{\circ}, 5.20 \times 10^{-4}$ nitrobenzene, $1.17 \times 10^{-4} \underline{\mathrm{M}}$ catechol, $5.94 \times 10^{-5} \underline{\mathrm{M}}$ Fe (III), $3.62 \times 10^{-3} \mathrm{M} \mathrm{H}_{2} \mathrm{O}_{2}, \mathrm{pH} 3.6$. Absorption based on nitrophenolate ion.

O. No cyclodextrin

Q. $1.26 \times 10^{-3} \underline{M}$ a-cyclodextrin Q. $1.10^{3} \times 10^{-3} \mathrm{M}$-cyclodextrin 
Figure 13: Dependence of yield on pH for treatment of acetanilid with cyclodextrin-modified

Hamilton system; $5.38 \times 10^{-4} \underline{M}$ acetanilid,

$1.17 \times 10^{-4} \underline{M}$ catechol, $5.94 \times 10^{-5} \underline{M}$

$\mathrm{Fe}(I I I), 4.0 \times 10^{-3} \underline{\mathrm{M}} \mathrm{H}_{2} \mathrm{O}_{2}, 8.4 \times 10^{-4} \underline{\mathrm{M}} \beta-$

A cyclodextrin, reaction time 2 hrs, 4-AAP

thinish. 


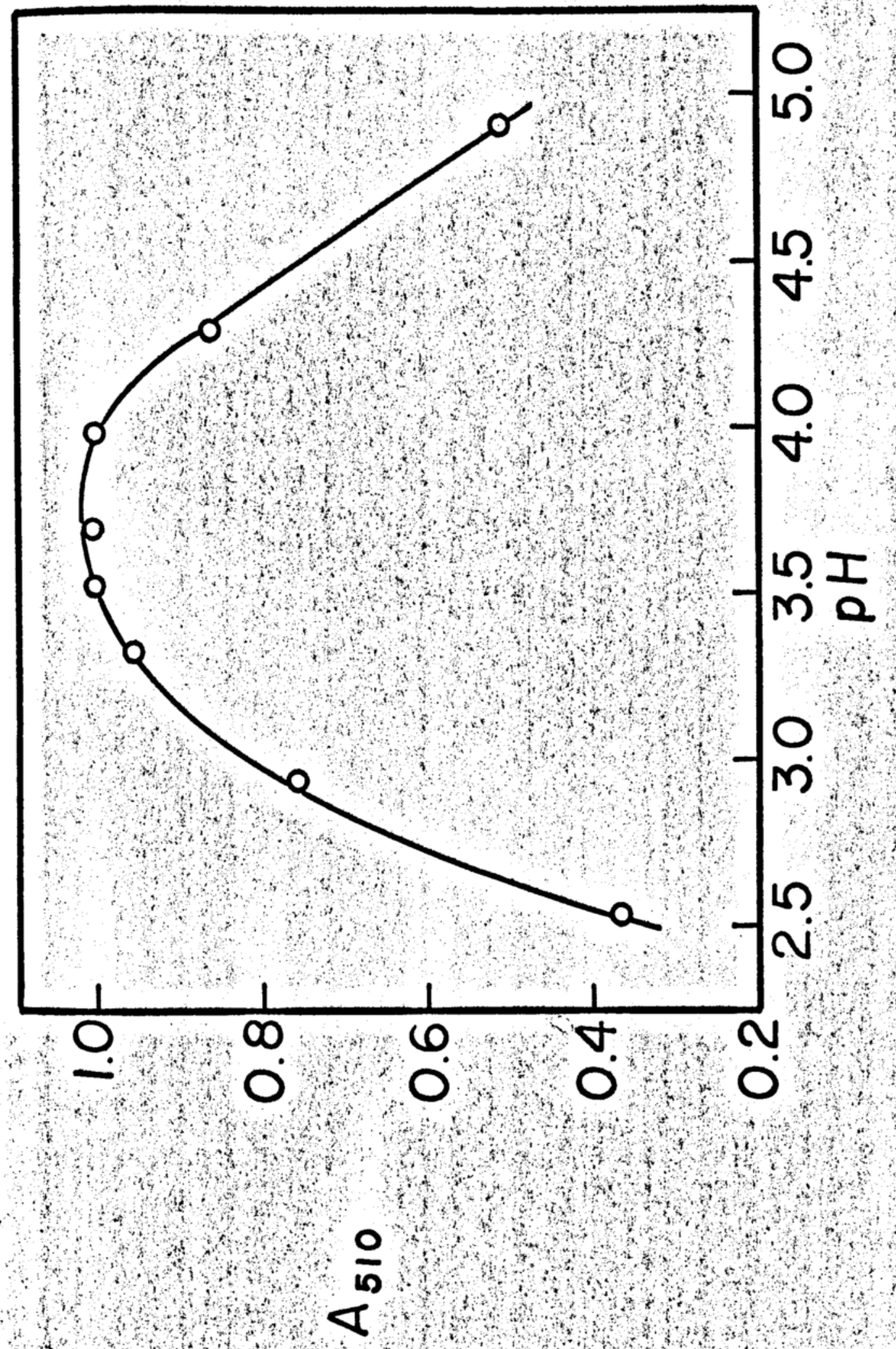


essentially pH independent over the range 3.5-4.0.

Hamilton (18) noted that the rate of oxidation of anisole was inhibited by increasing buffer concentration (presumably because the carboxylic acid effectively competes with the enediol for $F e($ III)). Figure 14 provides evidence that yield is also inhibited by increasing buffer concentration (see Discussion Section). In this study, the pH was, of course, constant.

\section{Analytical Results.}

Table $X$ lists the simple aromatic compounds that have been successfully treated by the cyclodextrin-modified Hamilton reagent. Linear working curves of absorbance against concentration were observed for most compounds up to concentrations of $8 \times 10^{-4} \underline{M}$. Fading of the color occurred with methyl benzoate and benzaldehyde, so the absorbance values were extrapolated to time of color development. (Nitrobenzene also exhibited fading, which appears to be associated with electron withdrawing groups. stability of the 4-aminoantipyrine-produced color will be discussed shortly.) Benzaldehyde gave a standard curve with marked negative curvature. Pyridine could not be determined because ㅇ- and p-hydroxy pyridines did not respond to the color development. The $\underline{m}$-substituted derivative does give a color. but apparently m-hydroxy pyridine was not a product of hydroxylation, or, if it was produced, its concentration in the final analytical solution was below detectable limits. 


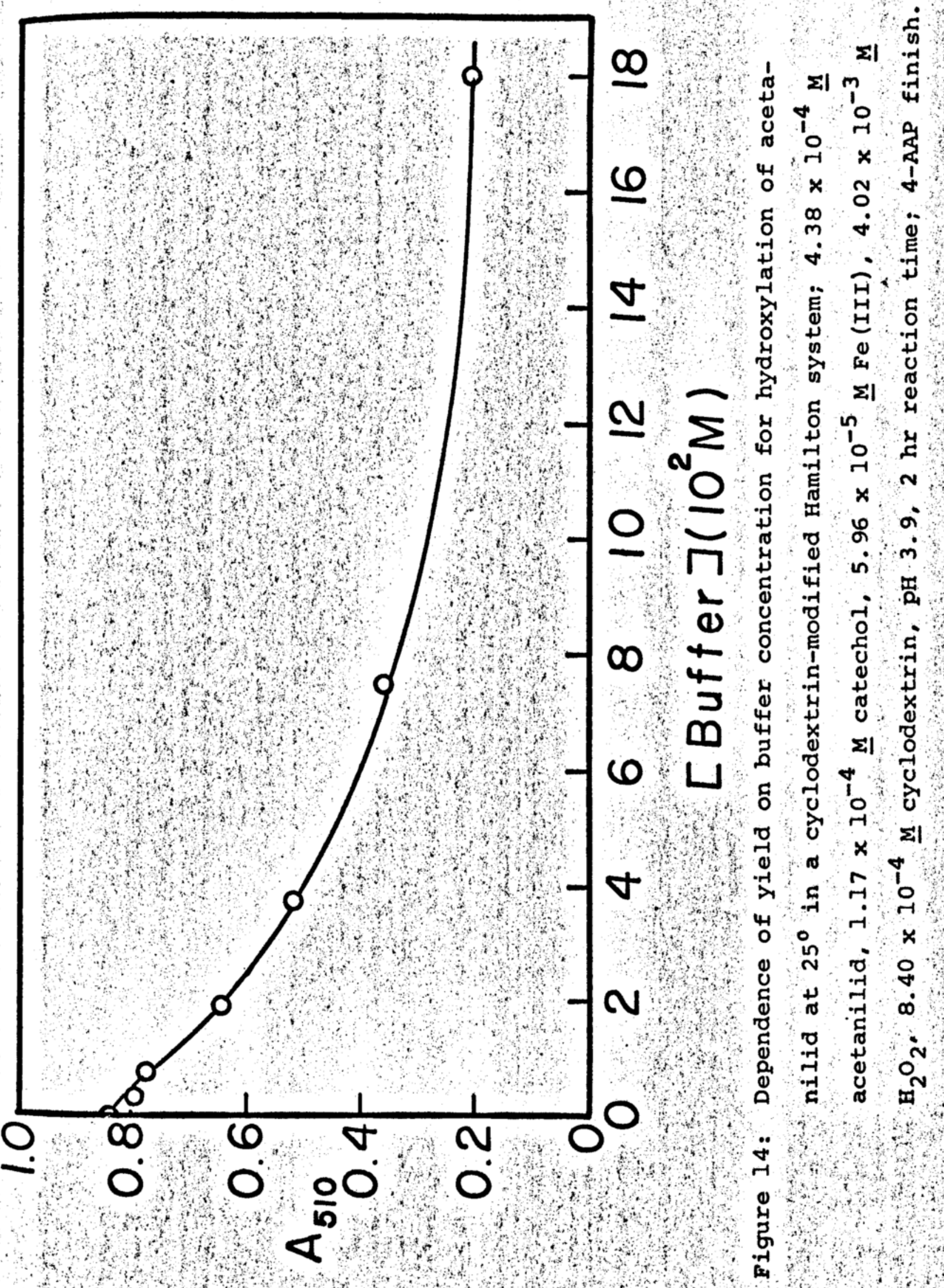


Table X

Aromatic Compounds Determined by the CyclodextrinModified Hydroxylation Procedure at $25^{\circ}$ a

Analytical

Reaction wavelength,

Sample time, $\mathrm{hr}$ nm $\mathrm{nm}^{\mathrm{b}}, 10^{-3} \mathrm{\epsilon}_{\mathrm{app}}$

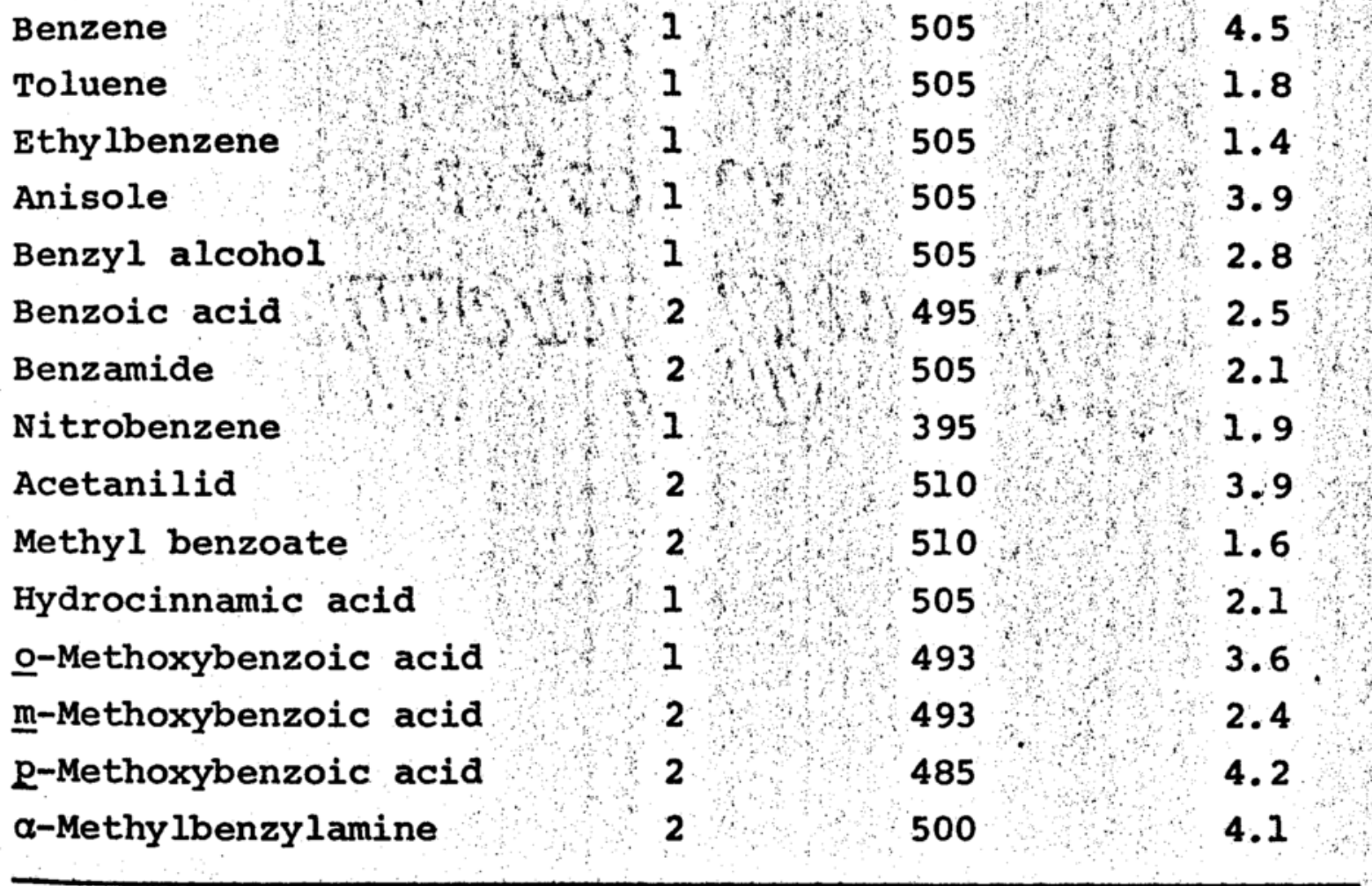

a $1.17 \times 10^{-4} \underline{M}$ catechol, $5.94 \times 10^{-5} \underline{\mathrm{Me}}$ (III), 3.0-4.0 $\mathrm{x}$ $10^{-3} \underline{\mathrm{M}} \mathrm{O}_{2}, 0.8-1.0 \times 10^{-3} \underline{\mathrm{M}}$-cyclodextrin, $\mathrm{pH} 3.6-4.0$.

b Color developed by the 4-aminoantipyrine method except with nitrobenzene, which was measured through the nitrophenolate absorption.

C Apparent molar absorptivity based upon total sample concentration, regardless of fate, in the final colorimetric solution. 
Aniline could not be determined either, because the hydroxylation product precipitated from solution. The choice of reaction time was based upon the nature of the time course curve, as previously discussed. In the determination of $6 \times 10^{-4} \mathrm{M}$ acetanilid, little or no interference was caused by $10^{-3} \mathrm{M}$ of methanol, acetonitrile, acetone, or ethyl acetate; N,Ndimethylformamide caused a $20 \%$ decrease in response, and dioxane, a $40 \%$ decrease. These data appear in Table XI. The reproducibility of the analytical method depends largely on solution preparation, time of reaction, and the usual spectrophotometric limitations. Under the reaction conditions described (see Table $\mathrm{x}$ ), the equation of the least-squares line for the hydroxylation of acetanilid (five concentration points, five trials per point) is given by Eq. (16)

$$
A_{510}=3.71 \times 10^{3} \mathrm{C}-0.016
$$

The standard deviation is 0.006 absorbance units.

Table XII contains a list of aromatic drugs and related compounds subjected to the procedure. Iinear working curves of absorbance against concentration were observed for most compounds up to concentrations of $8 \times 10^{-4}$ M. ${ }^{7}$ For samples containing electron witharawing groups (or substituents that

7 Above $8 \times 10^{-4} \mathrm{M}$, negative deviations from linearity resulted (see Discussion Section). 
Table XI

Effect on Yield of $10^{-3}$ M of Aliphatics for

the Cyclodextrin-Modified Hydroxylation

of Acetanilid at $25^{\circ}$.

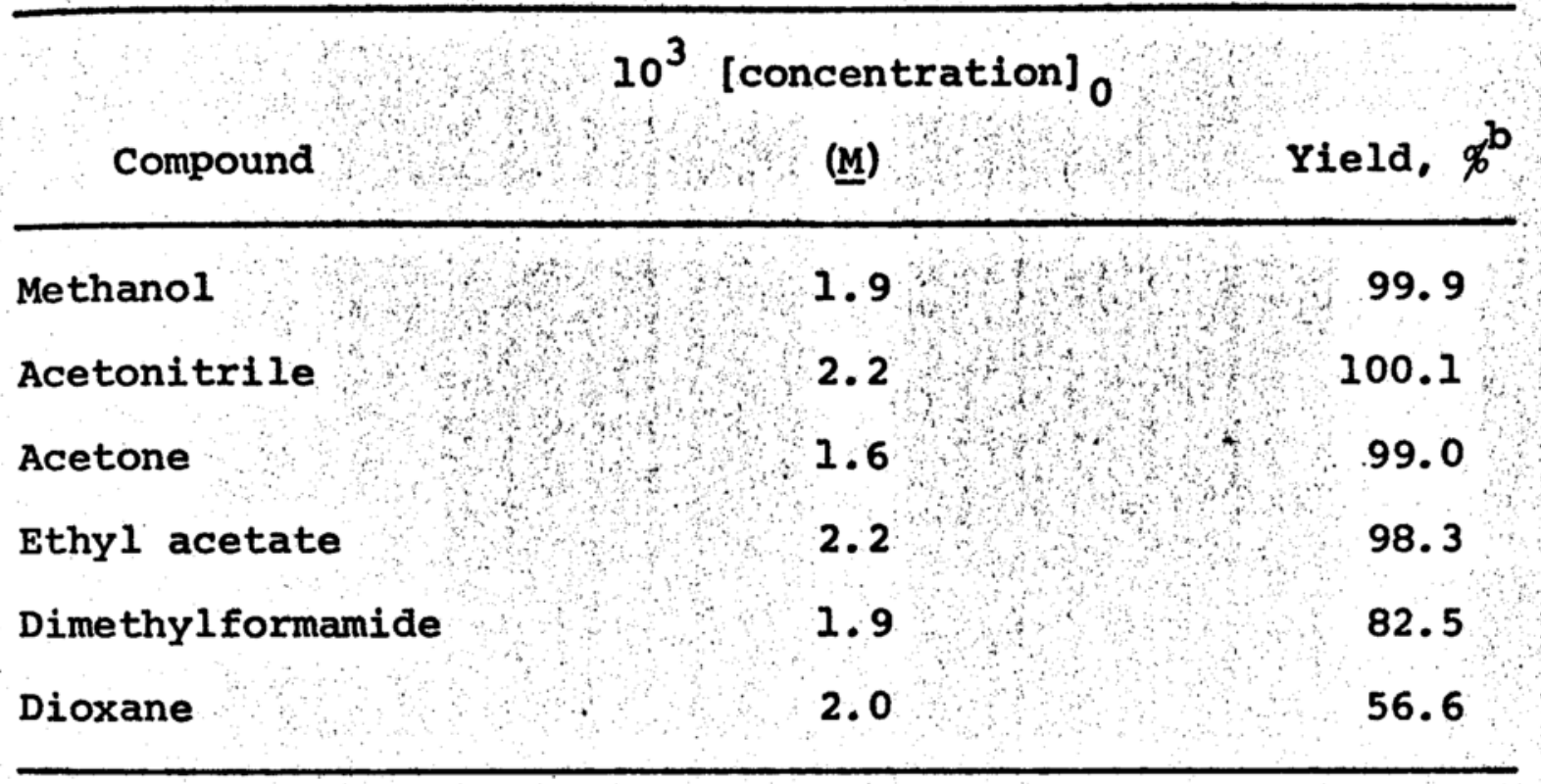

a $6.13 \times 10^{-4} \underline{M}$ acetanilid, $1.17 \times 10^{-4} \underline{M}$ catechol, $5.94 \times$ $10^{-5} \mathrm{M} \mathrm{Fe}(\mathrm{III}), 9.41 \times 10^{-4} \mathrm{M} \beta$-cyclodextrin, $3.60 \times 10^{-3} \mathrm{M}$ $\mathrm{H}_{2} \mathrm{O}_{2}, \mathrm{pH} 3.6,60$ min reaction time, 4-AAP finish.

b Yield relative to response for acetanilid in absence of aliphatic compounds. 
Table XII

Aromatic Drugs Determined by the CyclodextrinModified Hydroxylation Procedure at $25^{\circ}$.

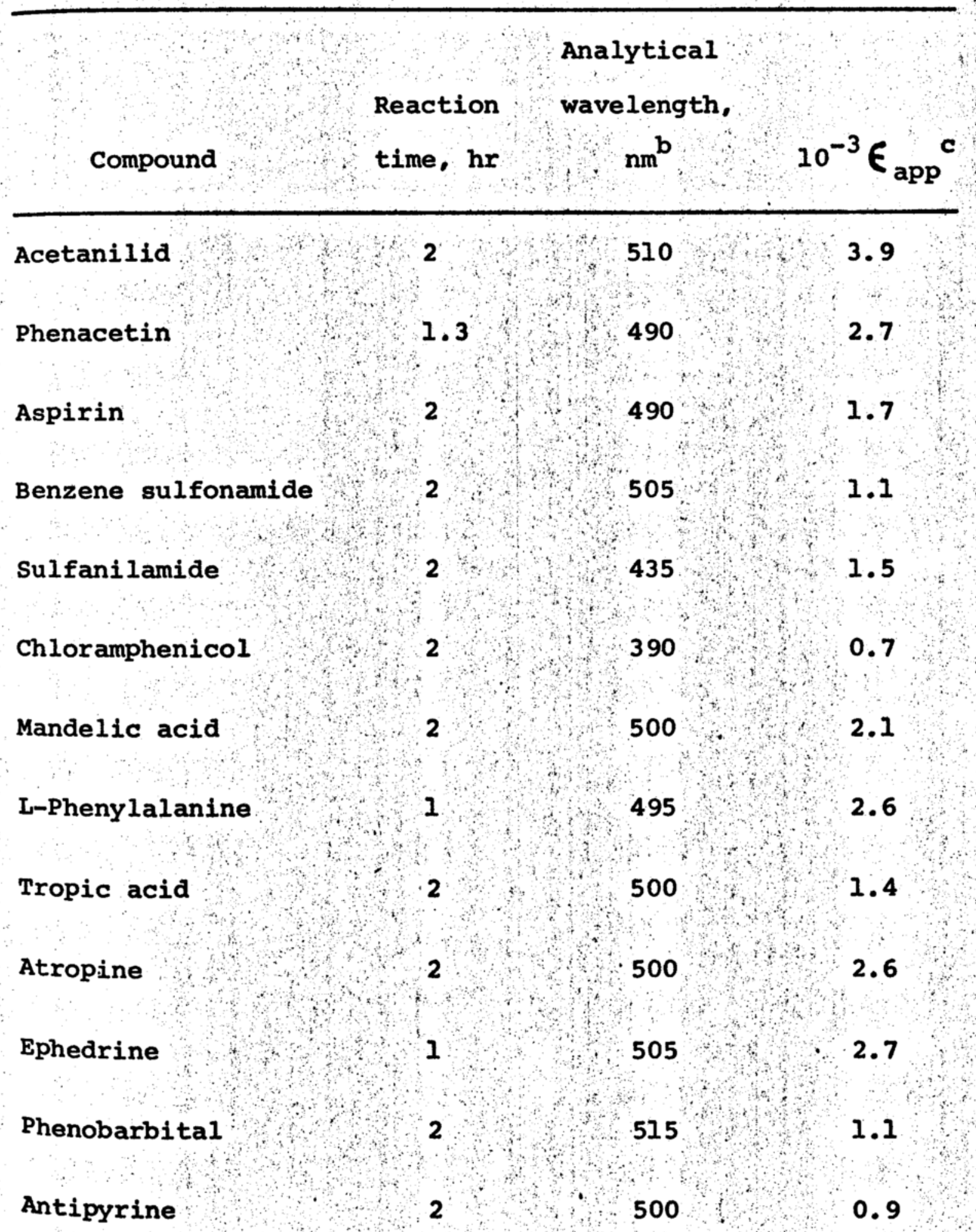


Table XII - cont.

Analytical

Reaction wavelength,

Compound time, $\mathrm{hr}, \mathrm{nm}^{\mathrm{b}}, 10^{-3} \epsilon_{\text {app }}{ }^{c}$

2-Naphthalene sulfonic acid

I-Tryptophan

Quinine

Physostigmine
2

2

2

2
510

485

490

495
0.6

0.5

0.5

0.2

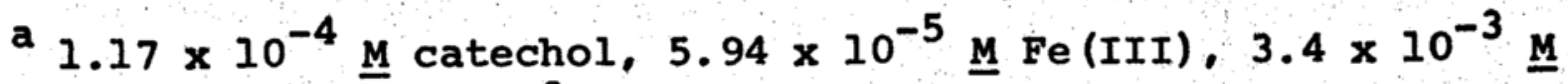
$\mathrm{H}_{2} \mathrm{O}_{2}, 1.0-1.3 \times 10^{-3} \mathrm{M}$-cyclodextrin, $\mathrm{pH} 3.6-4.0$.

b color developed by the 4-aminoantipyrine method except with sulfanilamide and chloramphenicol, which were measured through the nitrophenolate absorption.

C Apparent molar absorptivity based upon sample concentration, regardless of fate, in the final colorimetric solution. 
were oxidized to electron withdrawing groups ${ }^{8}$ ), where color fading occurred, absorbance values were extrapolated to time of color development. 9 Chlorpheniramine and benzalkonium chloride (zephiran) both gave standard curves with marked negative curvature. Benzocaine could not be determined because the hydroxylated product precipitated from solution. No interference was observed within $95 \%$ confidence limits, for the determination of acetanilid in the presence of tablet excipients at normal formulation levels. A typical synthetic mixture consisting of $35 \mathrm{mg}$ acetanilid, $100 \mathrm{mg}$ lactose, $10 \mathrm{mg}$ starch, $10 \mathrm{mg}$ talc, and $2 \mathrm{mg}$ stearic acid, gave an absorbance value of 0.690 .10 In the absence of excipients, an average absorbance (4 determinations) of 0.706 with a standard deviation of 0.005 was observed, and the range was 0.690-0.722 (student $t$ test, 95\% confidence limits, 3 degrees of freedom). Phenobarbital gave a standard curve exhibiting positive curvature, presumably because of an equilibrium

8

For example, the amine substituent of sulfanilamide apparently was oxidized to a nitro-group, enabling the nitrophenolate "color" to be used for quantification.

9

Acetanilid, mandelic acid, ephedrine, antipyrine, phenylalanine, chloramphenicol, physostigmine, benzenesulfonamide and sulfanilamide all exhibited color fading with the 4-AAP method.

10

The mixture, contained in a $50 \mathrm{ml}$ volumetric flask, was shaken with $30 \mathrm{ml}$ of water for $15 \mathrm{~min}$. After diluting to volume with water, a $2.0 \mathrm{ml}$ aliquot of the filtrate was subjected to the hydroxylation procedure as described in the Experimental Section. 
phenomenon within the cyclodextrin cavity (see Discussion section).

c. Hydroxylation with a Glucose-Modified Hydroxylating System

1. Stabilizing Effect of Glucose.

As a first step in investigating the stabilizing effect of cyclodextrins, the time course for hydroxylation of acetanilid as a function of glucose ( $\alpha-D-g l u c o s e, ~ d e x t r o s e)$ concentration was investigated. The results can be seen in Figure 15. It is apparent that glucose stabilizes the initial hydroxylation product, but its required concentration is about an order of magnitude greater than that for cyclodextrin.

Figure 16, showing a Beer's Law plot for the treatment of acetanilid with the Hamilton system in the presence and absence of glucose, reveals that indeed, a constant yield of monohydroxy product is obtained by incorporating glucose into the reaction mixture (filled circles); on the other hand, the curved absorbance-concentration plot in the absence of "stabilizer" (open circles) dramatically illustrates, again, the dependence of yield on the initial ratio of peroxide to substrate.

When cyclodextrin was incorporated into the Hamilton system as a stabilizer, phenobarbital gave a working curve with positive curvature while chlorpheniramine exhibited one 


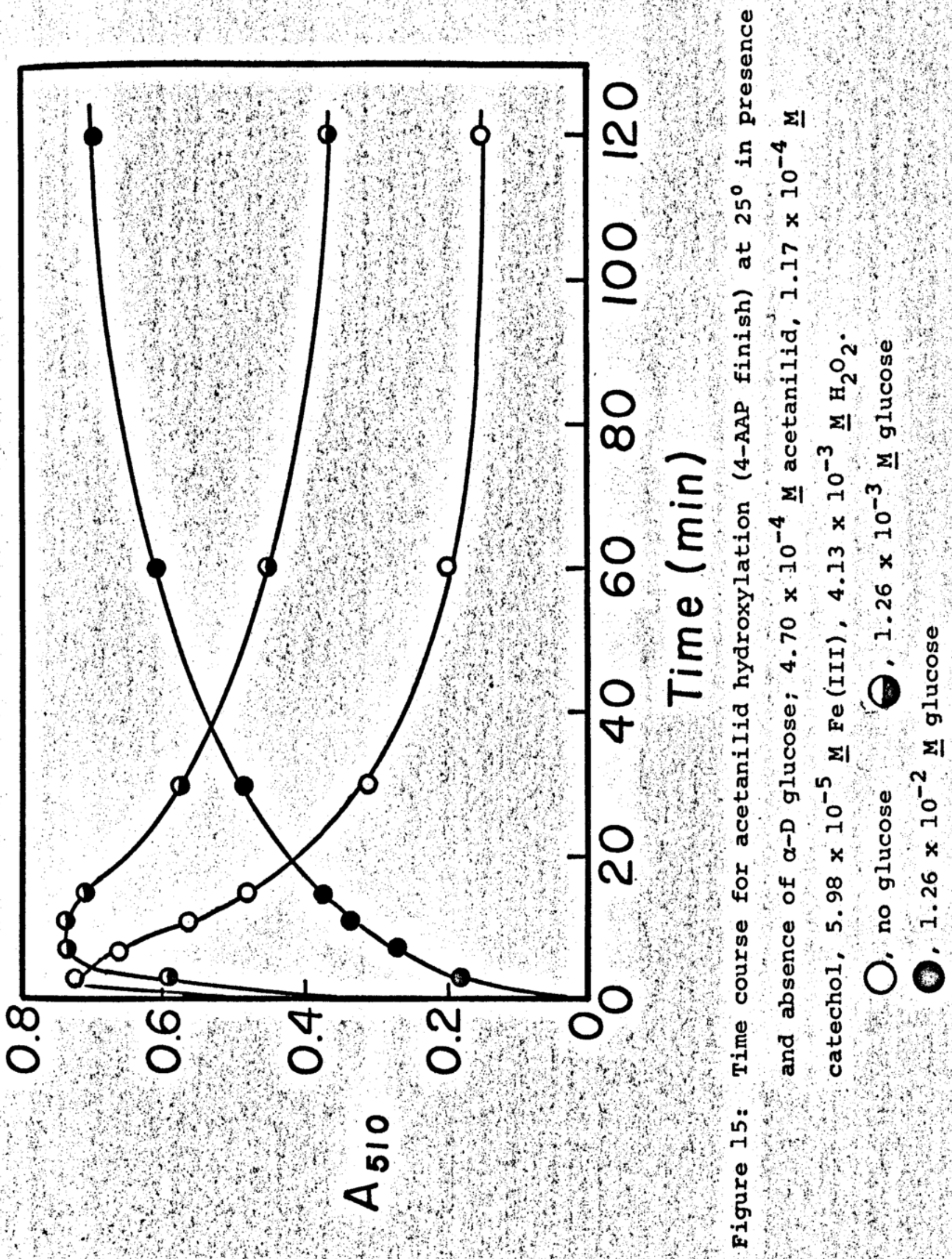




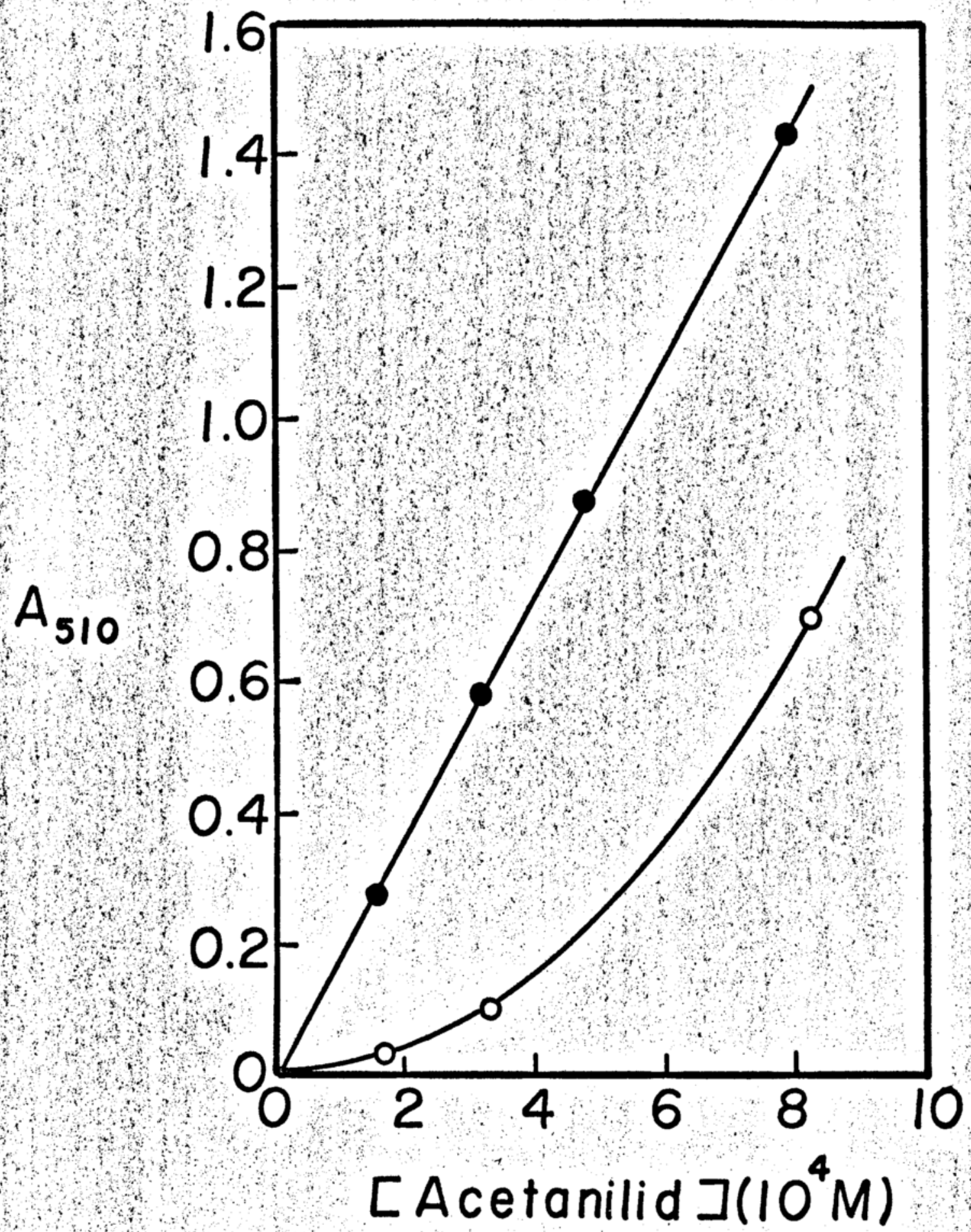

Figure 16, Concentration-response curve for hydroxylation of acetanilid at $25^{\circ}$ in the presence and absence of glucose; $1.17 \times 10^{-4} \underline{M}$ catechol, $5.98 \times 10^{-5} \underline{M}$ Fe (III), $4.13 \times 10^{-3}$ M $\mathrm{H}_{2} \mathrm{O}_{2}, 2 \mathrm{hr}$ reaction time; 4-AAP finish.

O. no glucose

Q. $7 \times 10^{-3}$ glucose 
with marked negative curvature. With glucose however, the working curve for phenobarbital was linear while that for chlorpheniramine showed less negative curvature. The effects, appearing graphically in Figures 17 and 18 respectively, may be associated with different mechanisms of stabilization (see Discussion Section).

\section{Temperature Effects.}

The effect of temperature on reaction rate was investigated in order to reduce reaction times from hours to minutes. ${ }^{11}$ Figure 19 illustrates the result of elevated temperatures $\left(55^{\circ}\right.$ and $\left.75^{\circ}\right)$ on the time course for hydroxylation of phenobarbital with a glucose-modified Hamilton system. At $55^{\circ}$ (filled circles), reaction time has been reduced from 2 hours at room temperature (see Table XII) to about 30 minutes, while at $75^{\circ}$ (half-filled circles) hydroxylation is complete in less than 10 minutes. Similar results at $75^{\circ}$, obtained for the treatment of acetanilid, are included in : Figure 19 to illustrate the generality of the temperature effect.

Inspection of Figure 19 also reveals that at higher temperatures, the yield for phenobarbital hydroxylation is depressed. Furthermore, under the conditions described, a phenobarbital standard curve showed slight negative curvature.

11 Temperature effects on a cyclodextrin-modified Hamilton system are probably more complex than with glucose because binding constants between cyclodextrin and aromatic are also temperature dependent (see Discussion Section). 


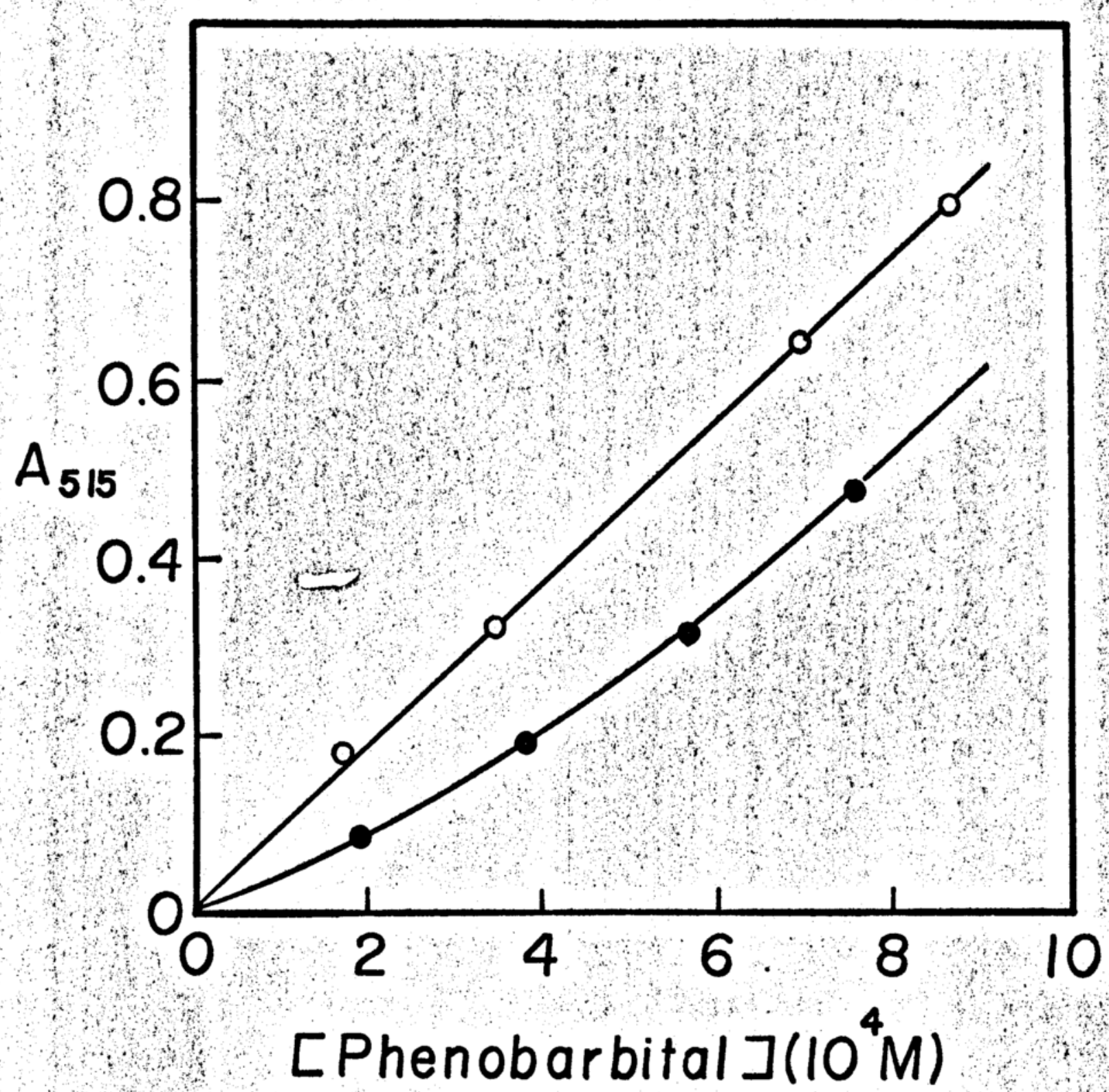

Figure 17: Concentration-response curve for treatment of phenobarbital with a modified Hamilton system at $25^{\circ} .1 .17 \times 10^{-4} \underline{\mathrm{M}}$ catechol, $5.94 \times 10^{-5} \underline{\mathrm{M}}$

Fe(III), $2 \mathrm{hr}$ reaction time, 4-AAP finish.

O. $7.24 \times 10^{-3} \underline{M}$ glucose; $3.90 \times 10^{-3} \underline{M}$ $\mathrm{H}_{2} \mathrm{O}_{2}$

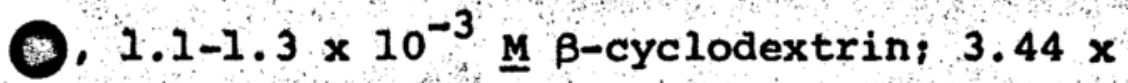
$10^{-3} \mathrm{M} \mathrm{H}_{2} \mathrm{O}_{2}$ 


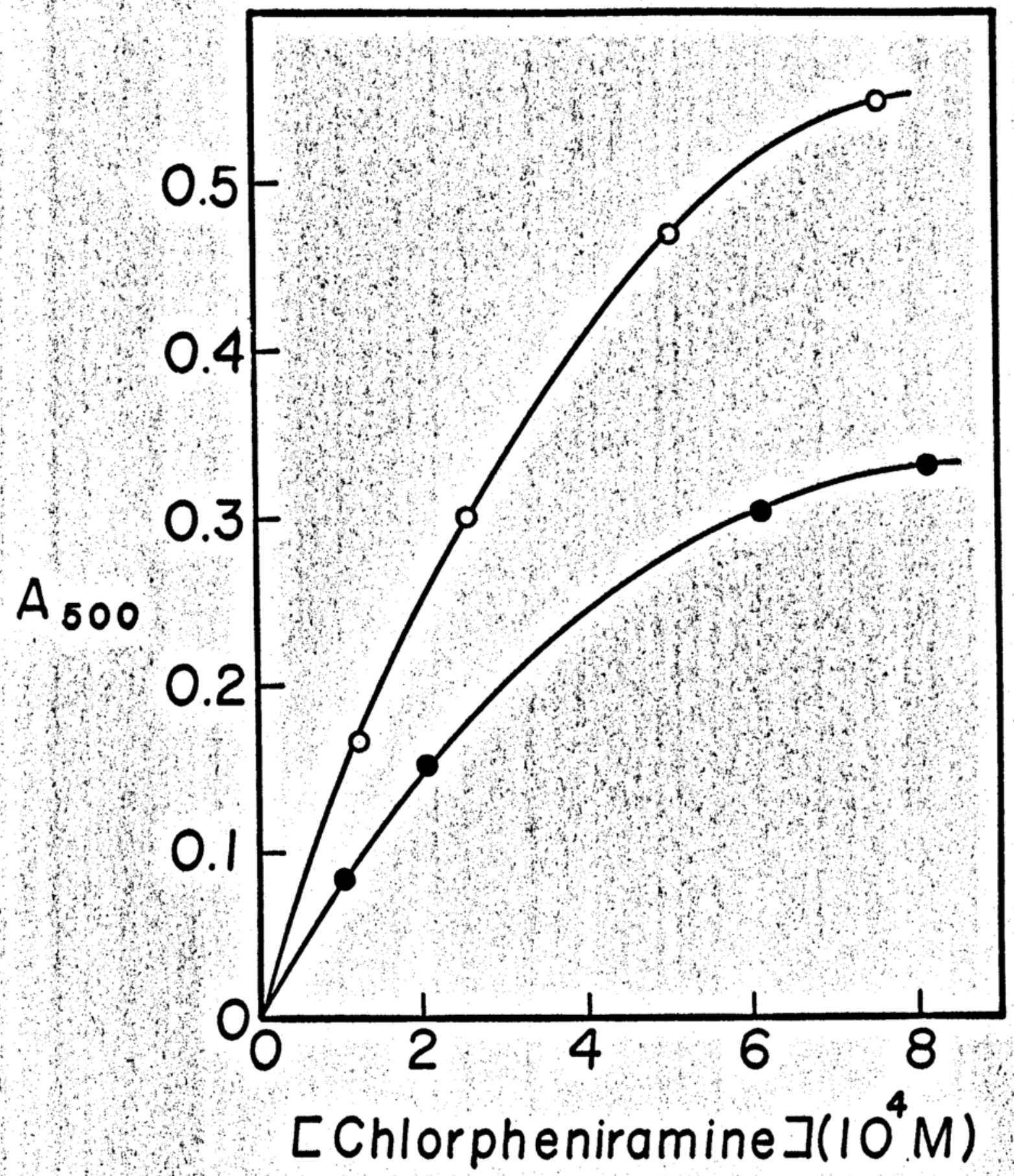

Figure 18: Concentration-response curve for treatment of chlorpheniramine with a modified Hamilton system at $25^{\circ}, 1.17 \times 10^{-4} \underline{M}$ catechol, $5.98 \times 10^{-5} \underline{M}$ Fe (III), $3.68 \times 10^{-3} \underline{\mathrm{M} \mathrm{H}} \mathrm{O}_{2}, \mathrm{pH} 3.9,2 \mathrm{hr}$ reaction time, 4-AAP finish.

O. $7.3 \times 10^{-3} \underline{M}$ dextrose

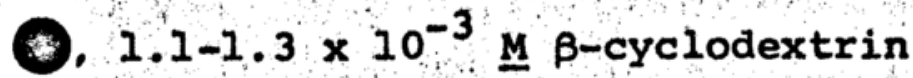


Figure 19: Effect of temperature on time course for treatment of acetanilide and phenobarbital with a glucose-modified Hamilton system; 1.17. $\times 10^{-4} \underline{M}$ catechol, $5.94 \times 10^{-5} \underline{M} \mathrm{Fe}(\mathrm{III})$, $3.90 \times 10^{-3} \underline{\mathrm{M}} \mathrm{O}_{2}, 7.24 \times 10^{-3} \mathrm{M}$ glucose; 4-AAP finish

Phenobarbital, $8.64 \times 10^{-4} \underline{M}$

Q. $55^{\circ}$

(1. $73^{\circ}$

Acetanilide, $5.94 \times 10^{-4} \underline{M}$

$74^{\circ}$ 


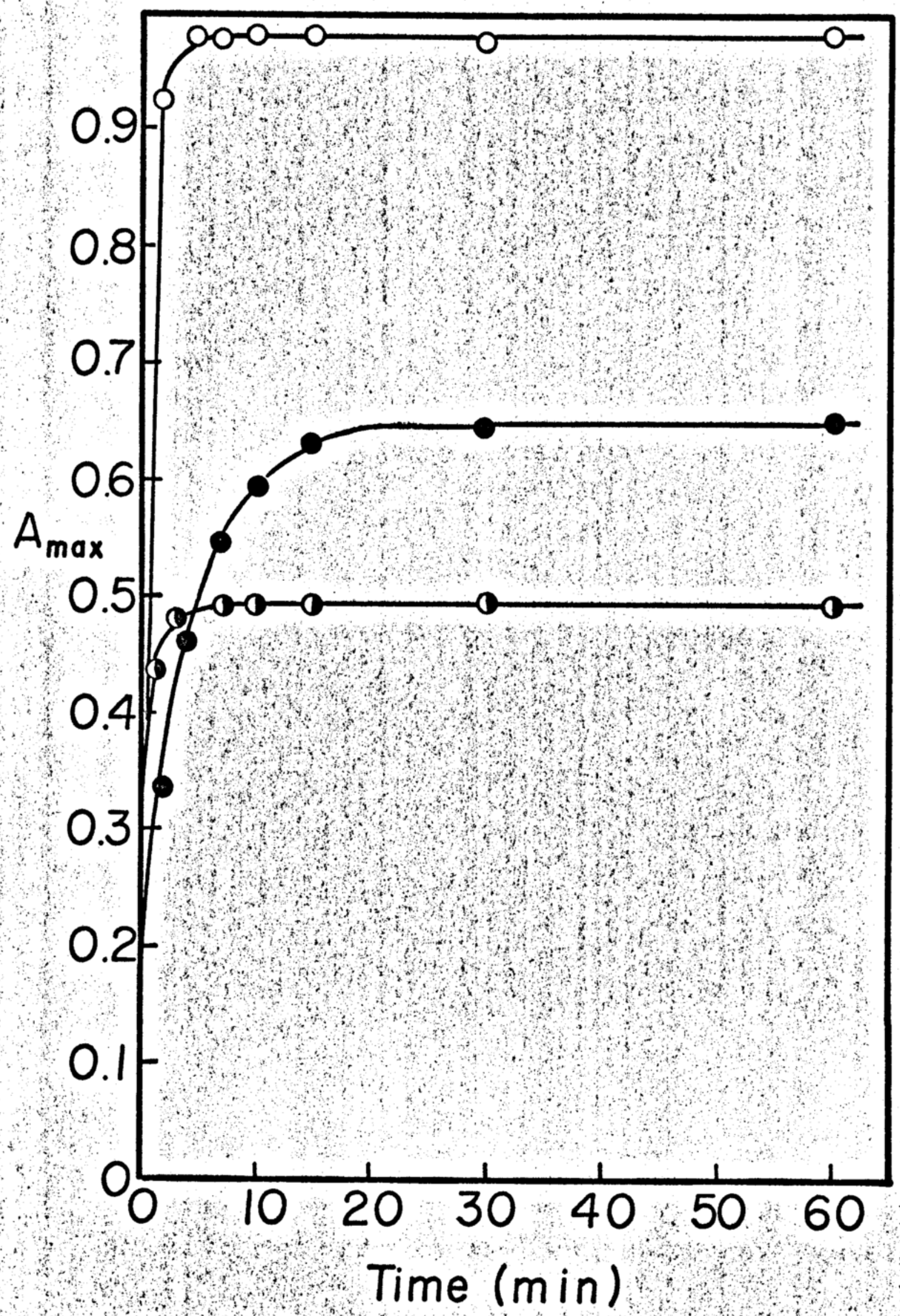


These data seem to suggest that at elevated temperatures, less glucose may be needed to stabilize the initial hydroxylation product; consequently the effect of glucose concentration on yield for the hydroxylation of phenobarbital, was studied. The data tabulated in Table XIII, show that the yield is glucose independent and maximal over a narrow range of stabilizer concentration $\left(2.20-3.67 \times 10^{-3} \underline{M}\right)$, and that at $7 \times$ $10^{-3} \mathrm{M}$ glucose (cf. Figure 19), the yield is depressed about $20 \%$ from its maximum value. When the standard curve for phenobarbital hydroxylation was re-run at lower glucose concentration $\left(3.67 \times 10^{-3} \mathrm{M}\right)$, it was linear. These data, graphically depicted in Figure 20, show the curvature in a Beer's Law plot at high stabilizer concentration (filled circles) and the linearity when glucose concentration was reduced (open circles). Also included is a standard curve at $25^{\circ}$, to illustrate the effect of temperature on yield, even though glucose concentration was optimized.

Table XIV summarizes data for the treatment of some monosubstituted aromatic compounds with the Hamilton system as a function of glucose concentration. The data are consistent with that for phenobarbital, displaying a narrow range of stabilizer concentration within which the yield is maximal.

\section{Dependence on Catechol.}

Hydroxylation with an unmodified Hamilton system revealed that a certain concentration of catechol (1.17 x $10^{-4} \underline{M}$ ) was necessary to obtain maximum yields of initial 
Table XIII
Effect of [Glucose] on Yield for the Hydroxylation of Phenobarbital at $75^{\circ}$ a

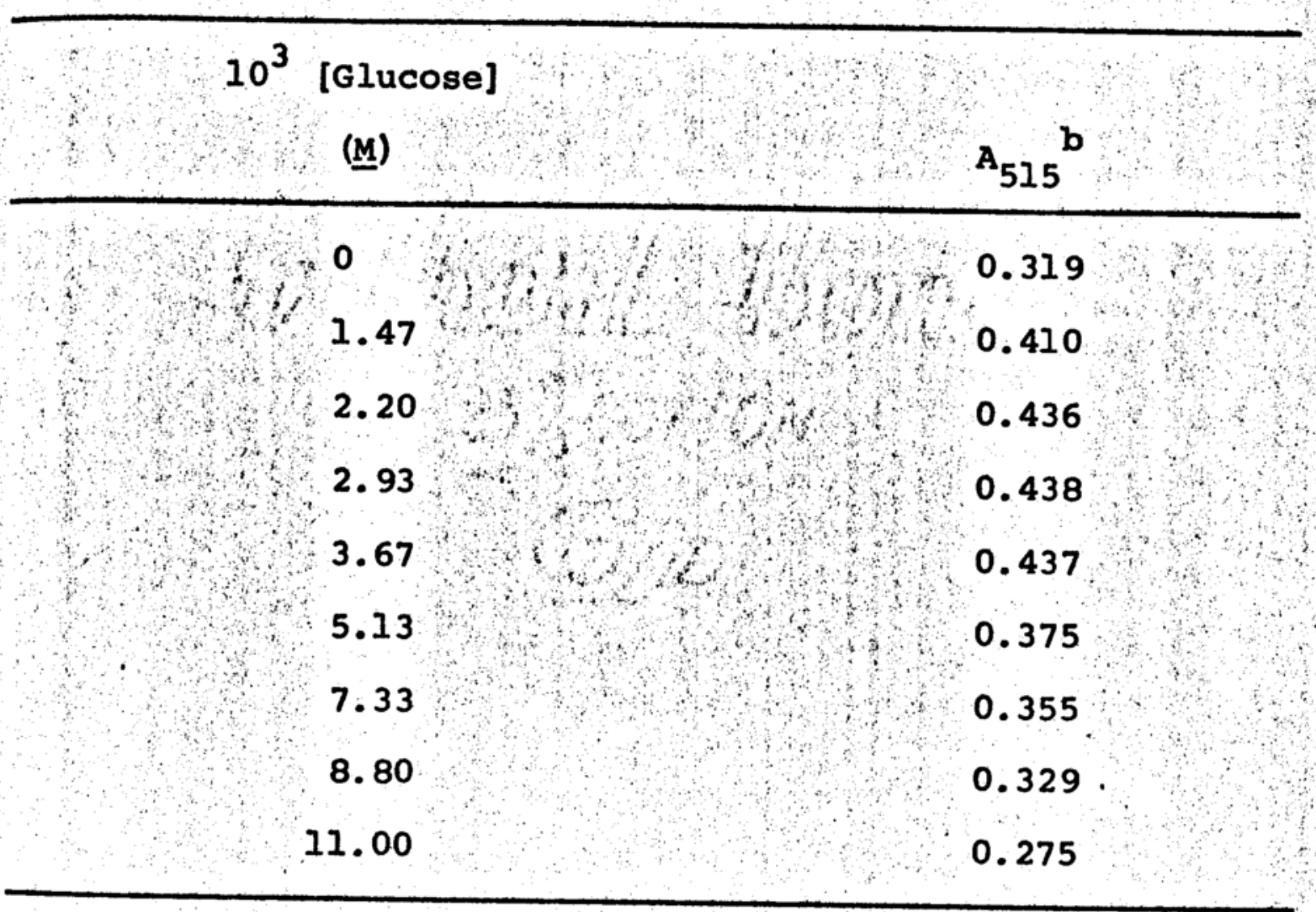

a $6.87 \times 10^{-4} \underline{M}$ phenobarbital, $1.17 \times 10^{-4} \underline{M}$ catechol, time. $94 \times 10^{-5} \underline{\mathrm{M} e}$ (III), $4.0 \times 10^{-3} \underline{\mathrm{M}} \mathrm{H}_{2} \mathrm{O}_{2}, \mathrm{IO}$ min reaction

b Absorbances corrected for blank; 4-AAP finish. 


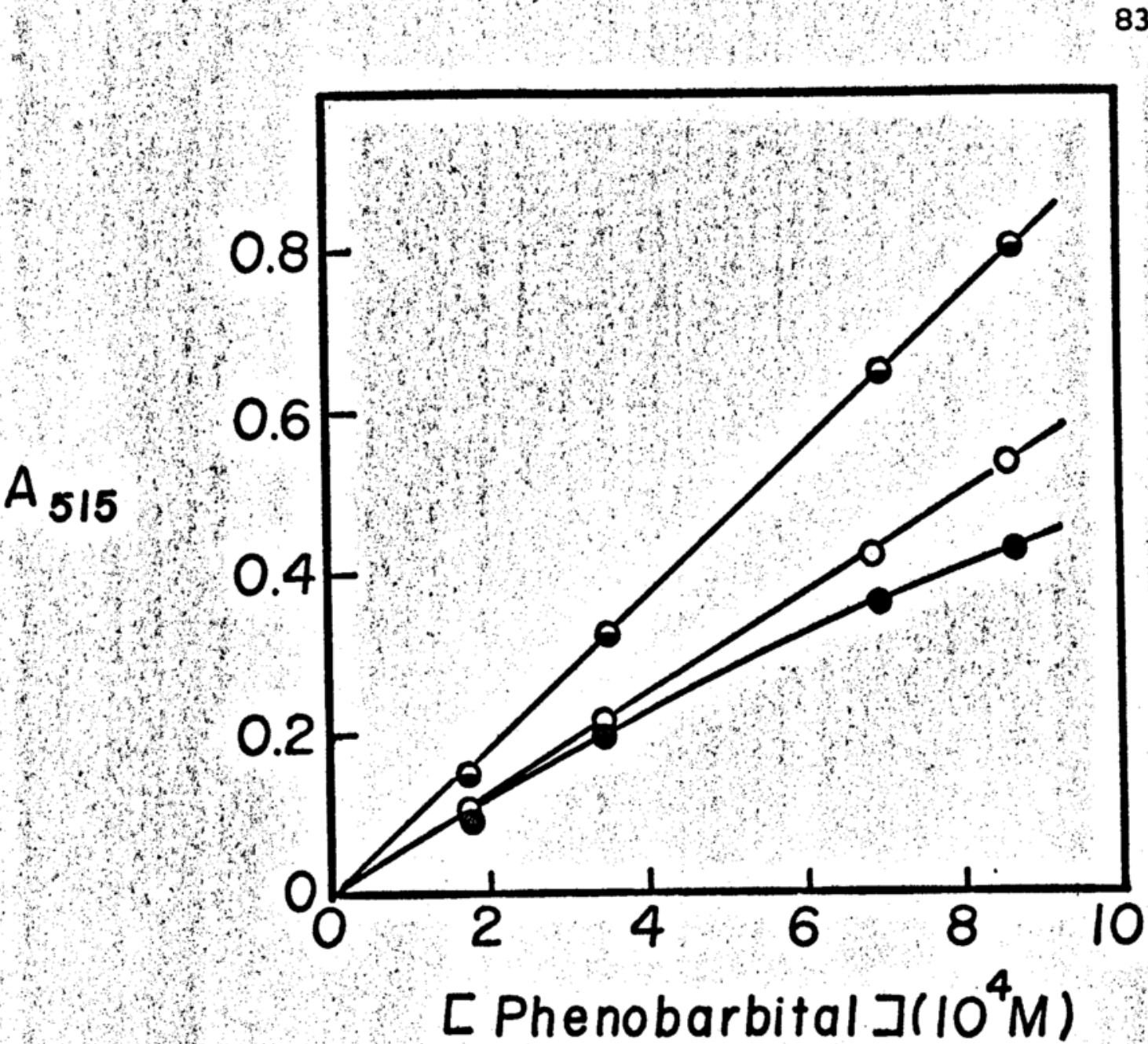

Figure 20. Concentration-response curves for treatment of phenobarbital with a glucose-modified Hamilton system; $1.17 \times 10^{-4} \underline{M}$ catechol, $5.94 \times 10^{-5} \underline{M}$

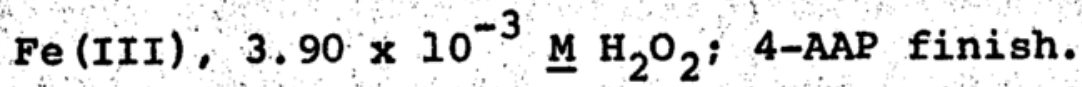

$0: 3.67 \times 10^{-3} \mathrm{M}$ glucose, $75^{\circ}, 10 \mathrm{~min}$ reaction time

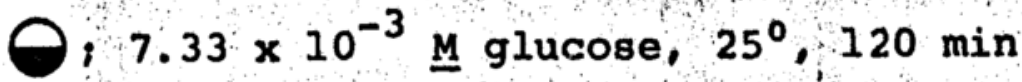
reaction time

$0,7.33 \times 10^{-3} \underline{M}$ glucose, $75^{\circ}, 10 \mathrm{~min}$ reaction time 
Table XIV

Dependence of Yield on [Glucose] for the Hydroxylation of Some Mono-substituted Benzene Derivatives at $75^{\circ}$ a

$10^{3}$ [Glucose]

(M)
$\mathrm{A}_{510}$

Acetanilid
Benzene $^{c}$

Anisolec

0.73

0.907

0.356

0.996

2.20

1.105

0.624

1.266

3.67

1.205

0.751

1.431

5.13

1.205

0.751

1.395

7.33

1.125

0.670

1.280

$a_{1.17 \times 10^{-4}} \underline{M}$ catechol, $5.94 \times 10^{-5} \underline{M} \mathrm{Fe}(\mathrm{III}), 3.90 \times$ $10^{-3} \underline{\mathrm{M}} \mathrm{H}_{2} \mathrm{O}_{2}, 10$ min reaction time; 4-AAP finish.

b $7.37 \times 10^{-4}$ M acetanilid.

C $6.07 \times 10^{-4} \underline{M}$ benzene.

d $6.87 \times 10^{-4} \underline{M}$ anisole. 
hydroxylation products. At $75^{\circ}$ with a glucose-modified Hamilton system, however, the yield is independent of catechol concentration above $1 \times 10^{-4}$ ‥ The data are presented in Figure 21.

\section{Analytical Results.}

Table XV lists the aromatic compounds that have been successfully treated by the glucose-modified Hamilton system at $75^{\circ}$. Linear working curves of absorbance against concentration were observed for most compounds at concentrations up to $1 \times 10^{-3}$ M. For solutions where color fading occurred, absorbance values were extrapolated to time of color development. Antipyrine gave a standard curve with marked negative curvature. Chlorpheniramine showed linear behavior up to $6 \times 10^{-4}$ M. No interference was observed for the determination of phenobarbital in the presence of tablet excipients at normal formulation levels. A typical synthetic mixture consisting of $65 \mathrm{mg}$ phenobarbital sodium, $26 \mathrm{mg}$ lactose, $20 \mathrm{mg}$ starch, $20 \mathrm{mg}$ talc, and $0.3 \mathrm{mg}$ stearic acid, gave an average recovery of $98.4 \% \pm 0.6 \%$ (four determinations) when compared against a standard phenobarbital solution carried through the same procedure. 12 Using a synthetic mixture

The synthetic mixture, contained in a $50 \mathrm{ml}$ volumetric flask, was shaken with $30 \mathrm{ml}$ water for $15 \mathrm{~min}$, the $\mathrm{pH}$ adjusted to 3.9 with $5 \% \mathrm{HCl}$, and the solution diluted to volume with water. A $4.0 \mathrm{ml}$ aliquot of the filtrate was subjected to the hydroxylation reaction as described in Experimental Section. 


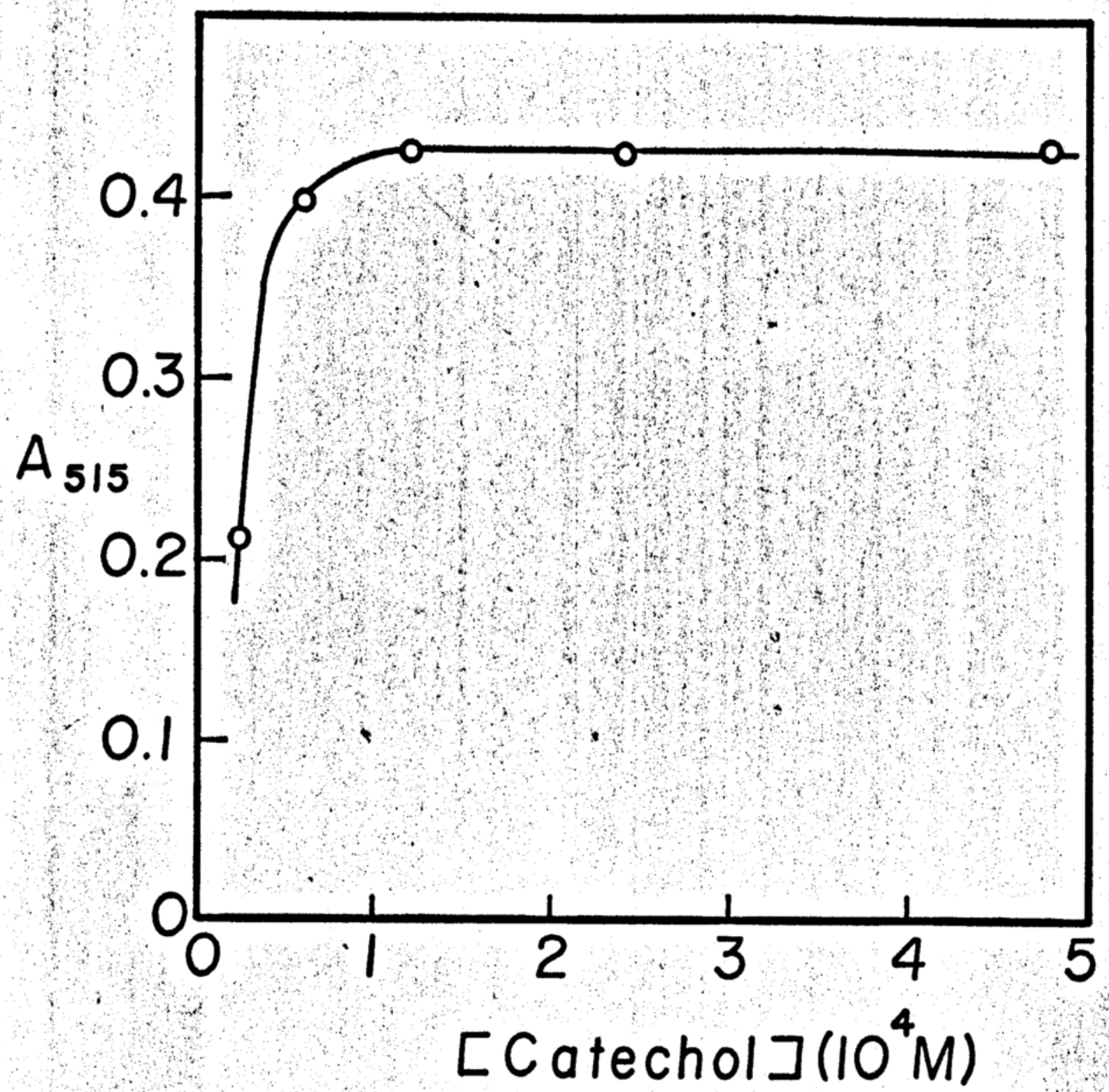

Figure 2.: Dependence on [catechol] of yield for hydroxylation of phenobarbital with a glucose-modified Hamilton system at $75^{\circ}, 6.87 \times 10^{-4}$ M phenobarbital, $3.90 \times 10^{-3} \mathrm{M} \mathrm{H}_{2} \mathrm{O}_{2},[\mathrm{Fe}(\mathrm{III})] /[$ catechol] $=$ $0.5,3.65 \times 10^{-3} \underline{M}$ glucose, 10 min reaction time; 4-AAP finish. 


\section{Table XV}

Aromatic Compounds Determined by the GlucoseModified Hydroxylation Procedure at $75^{\circ}$ a

\section{Analytical}

Compound wavelength, $\mathrm{nm}^{\mathrm{b}}$ $10^{-3}$ app $^{\mathrm{c}}$

Benzene

Anisole

Acetanilid

Phenacetin

Benzoic acid

o-Methoxybenzoic acid

m-Methoxybenzoic acid

p-Methoxybenzoic acid

Mandelic acia

Atropine

Ephedrine

Phenobarbital

Chloramphenicol

Chlorpheniramine
505

505

510

490

495

493

493

485

500

500

505

515

390

500
2. 9

4.4

3.4

3.1

1.7

3.7

2.8

5.5

1.3

2.1

2.2

1.3

0.8

2. 7

a $1.17 \times 10^{-4} \underline{\mathrm{M}}$ catechol, $5.94 \times 10^{-5} \underline{\mathrm{M}} \mathrm{Fe}(\mathrm{III}), 3-4 \times 10^{-3} \underline{\mathrm{M}}$ $\mathrm{H}_{2} \mathrm{O}_{2}, 3.7 \times 10^{-3} \mathrm{M}$ glucose, $\mathrm{pH} 3.6-4.0,10$ min reaction time.

b color developed by the 4-aminoantipyrine method except with chloramphenicol which was measured through the nitrophenolate absorption.

C Apparent molar absorptivity based upon sample concentration. regardless of fate, in the final colorimetric solution. 
composed of $65 \mathrm{mg}$ sodium phenobarbital, $200 \mathrm{mg}$ lactose, 20 $\mathrm{mg}$ starch, $20 \mathrm{mg}$ talc and $0.3 \mathrm{mg}$ stearic acid, a $10 \%$ decrease in response resulted. However, when the phenobarbital was extracted from the tablet excipients prior to hydroxylation ${ }^{13}$. an average recovery of $98.8 \% \pm 1.2 \%$ (four determinations) was obtained.

\section{Other Stabilizers.}

Both lactose and sorbitol were found to be effective stabilizers for the initial hydroxylation product when acetanilid was treated with the Hamilton system at $75^{\circ}$. The data, appearing in Table XVI, show that a maximum yield is obtained within a narrow range of stabilizer concentration dependent upon the nature of the stabilizer chosen.

At $25^{\circ}$, the following aliphatic compounds also exhibited stabilizer capability, acetonitrile, $0.1 \mathrm{M}$; isopropyl alcohol, $10^{-2} \underline{M}$; dioxane, $10^{-3} \underline{M}$; acetone, $10^{-1} \underline{M}$. A typical time course curve for acetanilid hydroxylation with a stabilizer-modified Hamilton system is presented in Figure 22.

The synthetic mixture, contained in a $60 \mathrm{ml}$ separatory funnel, was shaken with $10 \mathrm{ml}$ water for $15 \mathrm{~min}$, acidified with hydrochloric acid, and extracted with one $20 \mathrm{ml}$ and three $15 \mathrm{ml}$ portions of chloroform. The chloroform extracts were filtered through glass wool into a $100 \mathrm{ml}$ round-bottom flask, and evaporated to dryness using a roto-evaporator. The residue was dissolved in acetate buffer, quantitatively transferred to a $100 \mathrm{ml}$ volumetric flask, and diluted to volume with buffer. An $8.0 \mathrm{ml}$ aliquot was subjected to hydroxylation. 
Table XVI

Dependence of Yield on Stabilizer Concentration

for the Treatment of Acetanilid with the Hamilton system at $75^{\circ}$.

Concentration Limits $\mathbf{b}^{\mathrm{b}}$

(M)

$A_{\max , 510^{C}}$

Stabilizer

$0.0036-0.0051$

1.21

Glucose

$0.0036-0.0051$

1.18

Sorbitol

$0.0022-0.0037$

1.21

Lactose

$0.12-0.20$

1.17

Acetonitrile

a $7.36 \times 10^{-3} \underline{M}$ acetanilid, $1.17 \times 10^{-4} \underline{M}$ catechol, $5.94 \times$ $10^{-5} \underline{\mathrm{M} e}(\mathrm{III}), 4 \times 10^{-3} \underline{\mathrm{M}} \mathrm{H}_{2} \mathrm{O}_{2}, 10 \mathrm{~min}$ reaction time.

b

Concentration limits within which yield appears independent of stabilizer concentration.

C Absorbance corrected for blank; 4-AAP finish. 


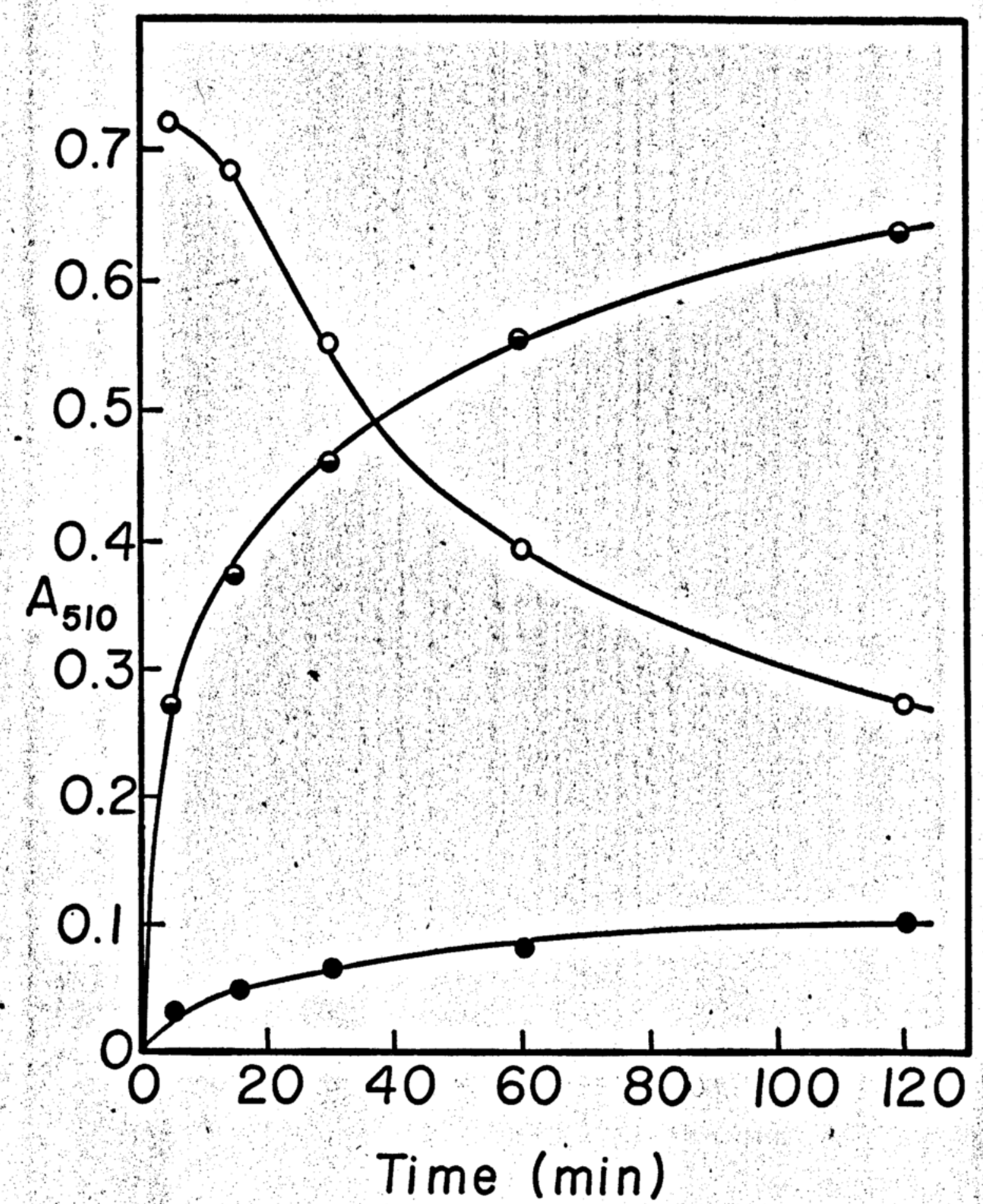

Figure 22: Treatment of acetanilid with an isopropyl alcoholmodified Hamilton system at $25^{\circ}, 5.05 \times 10^{-4} \mathrm{M}$ acetanilid, $1.17 \times 10^{-4} \underline{\mathrm{M}}$ catechol, $5.94 \times 10^{-5} \underline{\mathrm{M}}$

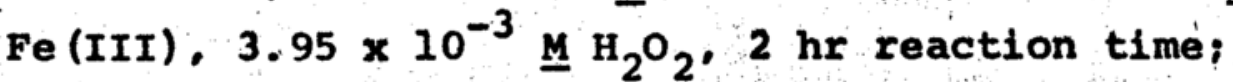
4-AAP finish.

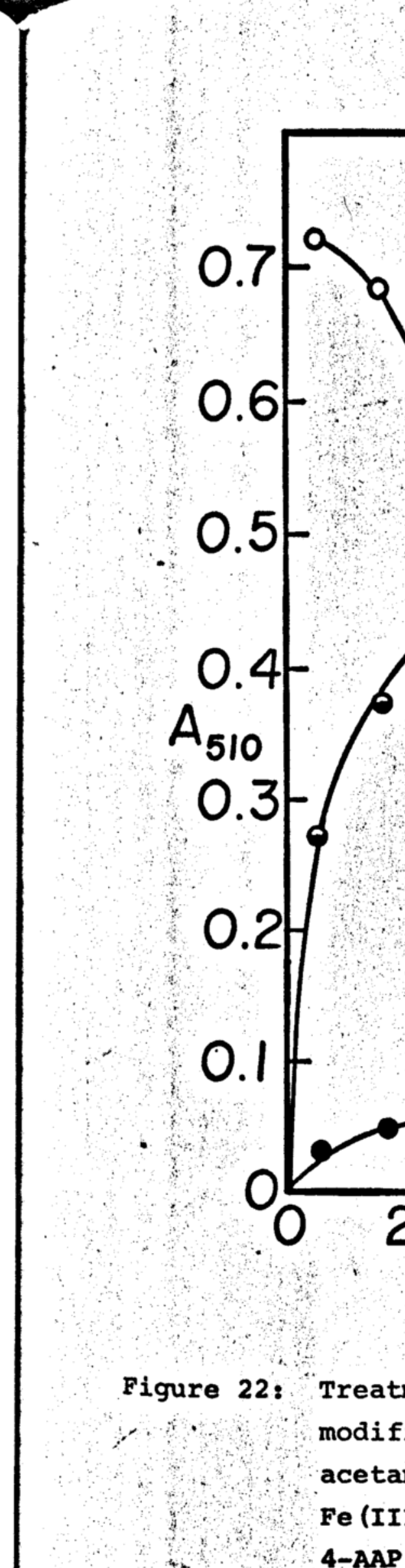

O. $10^{-3} \mathrm{M}$ isopropanol

O. $10^{-2} M$ 1sopropanol

3. $10^{-1} \underline{M}$ isopropanol 


\section{Studies on the Analytical Finish}

1. Stability of the 4-Aminoantipyrine-Produced Color.

The stability of the 4-aminoantipyrine-coupled dye has been the subject of several investigations $(33,35,37,39)$. The color was shown to be unstable for $\mathrm{pH}^{\prime} \mathrm{s}$ greater than 10 (39). and fading was accelerated when the dyes were exposed to strong sunlight (35). Recently, Svobodova, et al. (39) have associated color instability with the presence of electron-withdrawing groups.

Figure 23 shows the effect of substituents on color stability for some mono-substituted phenols subjected to the 4-aminoantipyrine finish in the present investigation. It is apparent that the presence of electron-withdrawing groups on the phenol molecule de-stabilizes the color. Phenol, on the other hand, produced a color stable for up to 3 hours.

Table XVII compares the extent of color loss for some

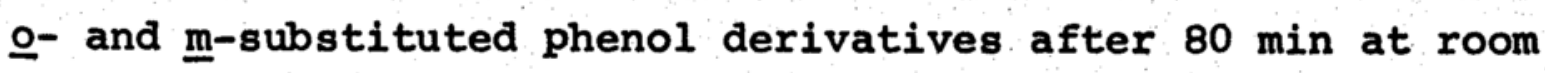
temperature. 14 The table reveals that color fading is most extensive for strongly electron-withdrawing groups in the ortho position. No explanation based upon structure-reactivity is apparent for the magnitude of the de-stabilizing effect exhibited by o-aminophenol, unless the amine group was partially oxidized by potassium ferricyanide to a nitroso or nitro group. is expelled during reaction. 


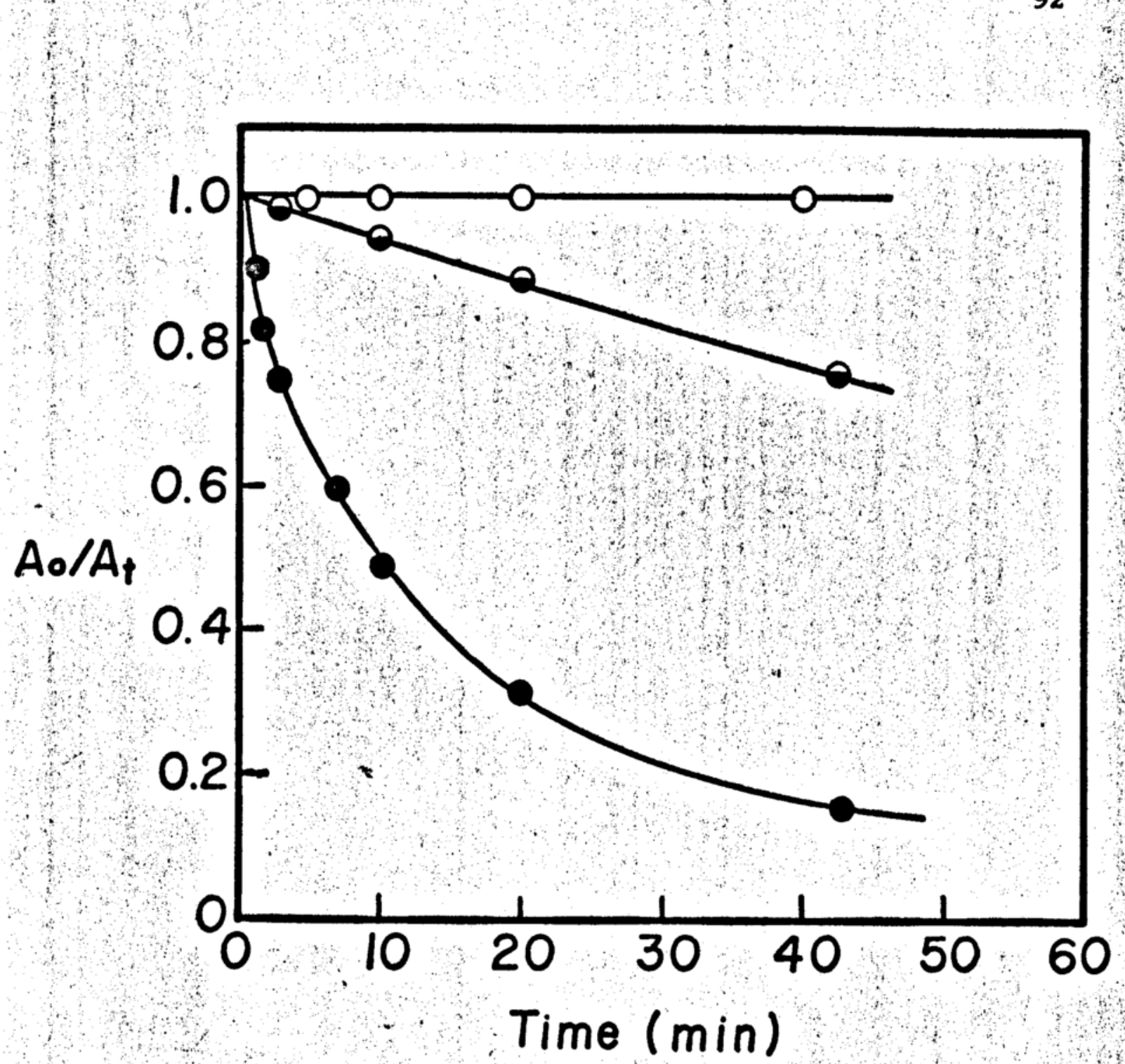

Figure 23. Effect of substituent on color stability of the antipyrine dye for some o-substituted phenols at $25^{\circ}$ in borate buffer.

substituent:

O. $\mathrm{H}$

○. $\mathrm{COCH}_{3}$

*. CHO 
Table XVII

Stability of the 4-Aminoantipyrine-Produced Color for Some Mono-Substituted Phenol Derivatives In Borate Buffer after $80 \mathrm{~min}$ at $25^{\circ}$.

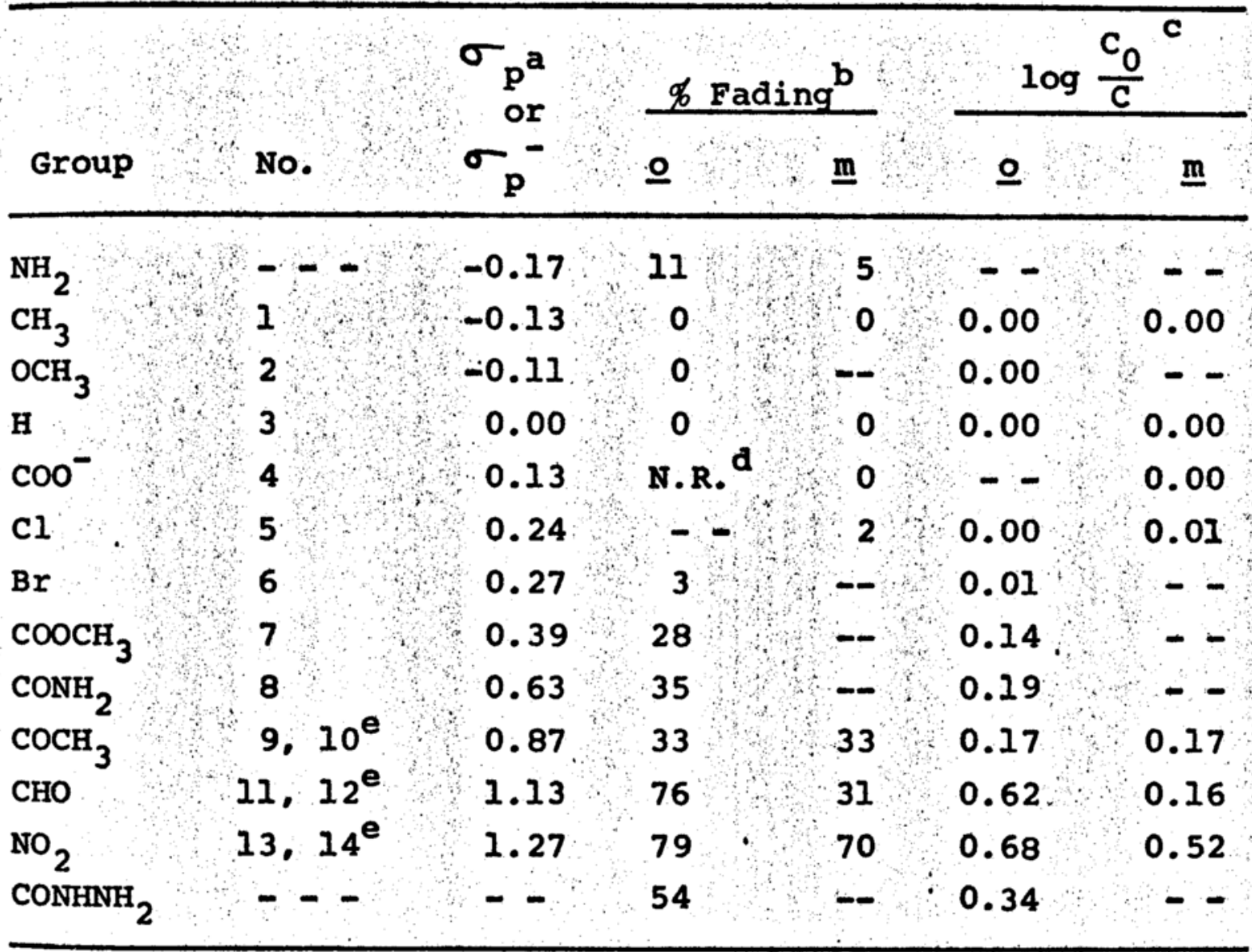

a $\sigma$ values are taken from March (95).

b of Fading $=\frac{A_{0}-A_{80}}{A_{0}} \times 100$ where $A_{0}$ represents the absorbance value at $510 \mathrm{~nm}$ in $1 \mathrm{~cm}$ cells extrapolated to time of color development, and $A_{80}$, the absorbance after 80 min.

c $\log \frac{C_{0}}{C}=\log \left(\frac{100-\not 6 \text { Fading }}{100}\right)$, see text.

d No reaction.

Numbers 10,12 and 14 represent the m-substituent. 
The extent of color fading is given by Eq. (17),

$$
\phi F=100\left(\frac{c_{0}-c}{c_{0}}\right)
$$

where $C_{0}$ is the concentration of antipyrine dye at time zero, and $c$, the concentration remaining at time t. Rearranging

Eq. (17) and taking its logarithm gives

$$
-\log \frac{C_{0}}{C}=\log \left(\frac{100-\% F}{100}\right)
$$

If, as a first approximation, first-order behavior is assumed, then

$$
\log \frac{\mathrm{C}_{0}}{\mathrm{C}}=\mathrm{kt}
$$

Substituting Eq. (18) into Eq. (19) and taking a constant time interval gives Eq. (20).

$$
-\log \left(\frac{100-\not F}{100}\right)=k^{\prime}
$$

A plot of $k^{\prime}$ against the Hammett $\sigma_{p}$ or $\sigma_{p}^{-}$substituent constant values 15 listed in Table XVII should reveal whether a correlation exists between color fading and the electrical effects of the substituents. The plot, appearing in Figure 24,

15

$\sigma_{\mathrm{p}}^{-}$values are used for substituents carrying an unshared pair of electrons which are capable of strong electronaccepting resonance; $\sigma_{p}$ values are used for the other 


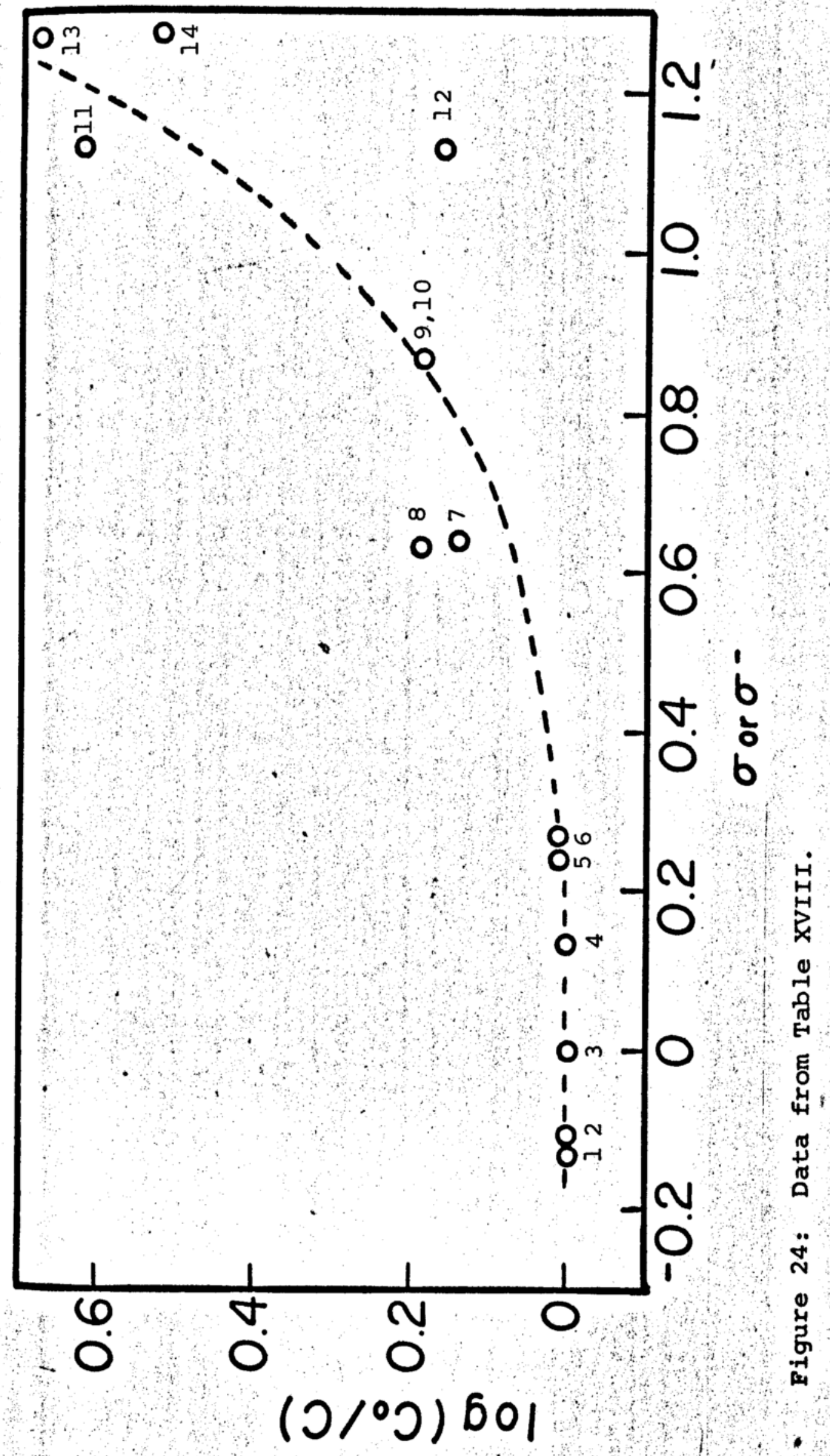


shows, indeed, that electron withdrawal facilitates the reaction. The qualitative nature of this correlation is obvious; nevertheless the plot indicates clearly that color instability is to be expected with phenols carrying substituents whose sigma values are greater than about 0.3 .

Extraction of the antipyrine dye into chloroform has been proposed to both concentrate and stabilize the color $(33,36,39) .16$ Table XVIII shows data consistent with the literature to illustrate that indeed, the red chloroform dye is more stable than that in aqueous solution.

\section{Other Finishes}

Oxidative coupling with both $\mathrm{N}, \mathrm{N}$-dimethyl-p-phenylenediamine (DPD) and 3-methyl-benzothiazolinone hydrazone (MBTH) were considered as alternatives to the 4-aminoantipyrine method for phenol determination. However, neither method produced a color when components of the Hamilton system were present. Further study revealed that peroxide was responsible for the interference. In addition, if the peroxide were destroyed prior to color development ${ }^{17}$, less than a normal response was obtained. However, lack of reproducibility made quantification difficult, consequently, the methods were not investigated further.

The molecule must be neutral to be extractable into chloroform, or, if any ionizable groups are present, they must be expelled during coupling.

17

Destruction of peroxide was accomplished by adding triethylenetetramine to the reaction solution (96). 
Table XVIII

Stability of the 4-Aminoantipyrine-Produced color

for Some o-Substituted Phenol Derivatives in

Borate Buffer and in Chloroform

after $40 \mathrm{~min}$ at $25^{\circ}$.

\begin{tabular}{cccc}
\hline & & \multicolumn{2}{c}{ \% Fading } \\
\cline { 3 - 4 } & Substituent & $\mathrm{CHCl}_{3}$ & $\mathrm{H}_{2} \mathrm{O}$ \\
\hline $\mathrm{CHO}$ & 44 & 70 \\
\hline $\mathrm{COCH}_{3}$ & 0.5 & 18 \\
$\mathrm{CONH}_{2}$ & 0.6 & 16 \\
\hline
\end{tabular}

a $\%$ Fading $=\frac{A_{0}-A_{40}}{A_{0}} \times 100$ where $A_{0}$ represents the absorbance value at $510 \mathrm{~nm}$ in $1 \mathrm{~cm}$ cells extrapolated to time of color development, and $A_{40}$. the absorbance after $40 \mathrm{~min}$. 
IV. DISCUSSION

A. The Unmodified Hamilton System

The Hamilton system afforded a means of converting aromatic compounds to phenols rapidly and in high yield when compared with other aqueous hydroxylating systems (see Table I). Consequently, the application of this system to the quantitative determination of some aromatic compounds was investigated.

Hamilton (17-19), measuring the kinetics of loss of peroxide when the aromatic substrate was in excess, found that the reaction rate was decreased by high buffer concentration, high catechol concentration ${ }^{1}$, and by the presence of organic solvents such as acetone or ether. In our studies, we found that the yields of monohydroxy product were similarly depressed.

Hamilton $(17,18)$ indicated that although reaction rates were independent of $\mathrm{pH}$ from $3.5-4.2^{2}$, the total yield of phenols (based upon peroxide) increased at lower pH outside this range (19). ${ }^{3}$ Thus, the yield of phenol from benzene was

1 Formation of inactive (or less active) iron-catechol complexes at high catechol concentration was proposed to explain the rate decrease.

2

Rate constants were extrapolated at different $\mathrm{pH}$ 's to zero buffer concentration.

3

More rapid destruction of catalyst (enediol) at higher pH was proposed to explain the apparent dependence of yield on $\mathrm{pH}$. 
$52 \%$ at $\mathrm{pH} 2.9$ and $28 \%$ at $\mathrm{pH} 4.2$; that for nitrobenzene was $42 \%$ at $\mathrm{pH} 2.9$ and $27 \%$ at $\mathrm{pH} 4.2$. We observed, however, that under conditions of maximum yield $\left(\left[\mathrm{H}_{2} \mathrm{O}_{2}\right]_{\alpha} / \text { Substrate }\right]_{0}=2$; $[\mathrm{Fe}(\mathrm{III})] /\left[\right.$ Catechol] $\left.=0.5 ;[\text { Catechol }]_{0}=1.2 \times 10^{-4} \mathrm{M}\right)$. yields of monohydroxy product were depressed at $\mathrm{pH} 2.9$ and were $\mathrm{pH}$ independent and maximum in the range 3.5-4.2.

The apparent discrepancy noted above is probably due to differences in the initial condition. Hamilton's yield calculations were based upon peroxide consumption with the aromatic in excess; ours, on the initial aromatic concentration when peroxide was in excess (i.e., peroxide is twice substrate concentration). Because of the non-specificity of the oxidizing agent, it should not discriminate between the aromatic substrate and its monohydroxy product (or catechol, for that matter). Catechol will ultimately be generated from benzene (and catechol will be destroyed by hydroxylation), but under Hamilton's conditions, its net concentration will increase because [Benzene] ${ }_{0} \gg$ [Catechol] $_{0}$. Consequently, the dependence of yield on pH observed by Hamilton must be related to the rate of hydroxylation of phenol at different $\mathrm{pH}$ 's.

We have found that the rate for hydroxylation of phenol is three times greater at $\mathrm{pH} 3.9$ than at 2.9. 4 The outcome (under Hamilton's conditions) would be a lower yield of the

4

The conditions were: $7.21 \times 10^{-4} \mathrm{M}$ phenol, $1.20 \times 10^{-4} \mathrm{M}$ catechol, $4.27 \times 10^{-5} \mathrm{M} F\left(\right.$ III), $1.82 \times 10^{-2} \mathrm{M} \mathrm{H}_{2} \mathrm{O}_{2}, 25^{\circ}$, no oxygen. $t_{I / 2}\left(\mathrm{pH}_{2.9}=5 \mathrm{~min} t_{1 / 2}(\mathrm{pH} 3.9)=1.5 \mathrm{~min}\right.$. 
monohydroxy product at $\mathrm{pH} 3.9$ (based upon the amount of peroxide that reacted), because at higher $\mathrm{pH}$ values, more catechol is generated, and high catechol concentration inhibits the loss of peroxide.

Under our conditions, the initial ratio of peroxide to substrate as well as initial catechol concentration, had been adjusted to give maximal yield values (based upon substrate). Moreover, these experiments were conducted in the $\mathrm{pH}$ range 3.5-4.2 where yield was essentially $\mathrm{pH}$ independent. Catechol will be generated from benzene and destroyed by hydroxylation (as under the Hamilton condition), but its net concentration will be such that maximal yields will result at the higher $\mathrm{pH}$ value. Thus, at pH 2.9, although less catechol is generated from phenol, its net concentration will be shifted from the optimum value and depressed yields will occur.

In the absence of stabilizer, when the ratio of peroxide to substrate is greater than 2, hydroxylation clearly continues beyond the introduction of the first hydroxy group (cf. Figure 1). (This occurs under other conditions also, but may not be obvious from the time course.) This sequential hydroxylation is taken to be approximately described schematically by Eq. (21),

$$
\mathrm{A} \stackrel{\mathrm{k}_{1}\left[\mathrm{H}_{2} \mathrm{O}_{2}\right]}{\longrightarrow} \text { B } \stackrel{\mathrm{k}_{2}\left[\mathrm{H}_{2} \mathrm{O}_{2}\right]}{\longrightarrow} \mathrm{C}
$$

where A represents the aromatic sample compound, B, the initial hydroxylation product, and $c$, subsequent hydroxylation products derived solely from B. Recall that with the 
quantitative measurement employed here (oxidative coupling with 4-aminoantipyrine), only B responds. Eq. (22) gives, then, the corresponding rate of change in $[B]$, and therefore in absorbance

$$
\frac{d[B]}{d t}=k_{1}[A]\left[\mathrm{H}_{2} \mathrm{O}_{2}\right]-k_{2}[\mathrm{~B}]\left[\mathrm{H}_{2} \mathrm{O}_{2}\right]
$$

since the reagent is non-selective (i.e., anisole and nitrobenzene are hydroxylated at similar rates), as a first approximation, $k_{1}=k_{2}$. Then, $d[B] / d t>0$ when $[A]>[B]$ (this is, incidentally, the initial condition), and the absorbance increases with time. When $[A]<[B]$ and provided that sufficient peroxide is present, $d[B] / d t<0$ and loss of the initial hydroxylation product is observed. Finally, $d[B] / d t=0$ when $[A]=[B]$ or when all of the peroxide is used up. Stable absorbance values are obtained when either condition is fulfilled (cf. Figure 2), however, the former represents the situation giving maximum yields at a ratio of peroxide to substrate about 2, while the latter gives lower yields at lower ratios because of the limited availability of peroxide.

Eq. (22) does not precisely describe the unmodified Hamilton system because the reagent is decomposing (by hydroxylation of catechol and concurrent decomposition of peroxide) during the course of the reaction. This "aging" phenomenon not only alters in some degree the catalytic effectiveness of the enediol, but it uses up peroxide. 
Ignoring for the moment dihydroxylation, which will decrease the yield of $B$ by depleting both $B$ and peroxide, the yields of monohydroxy product will be less than 100\% solely due to "aging" of the Hamilton system. As long as catechol must be present and as long as the $[\mathrm{A}] \mathrm{O} /\left[\mathrm{H}_{2} \mathrm{O}_{2}\right]$ o ratio is 2 or more. there is not enough peroxide to convert all of A to $B$.

Sequential hydroxylation places a theoretical limit on the maximum yield of $B$, since the value of $[B] /[A]_{0}$ is a function of the ratio of $k_{2} / k_{1}$. In the limit, as $k_{2} \rightarrow 0$, $[B] /[A]_{0} \rightarrow 1$. This ratio of rate constants $\left(k_{2} / k_{1}\right)$ should be determined by the electronic natures of $A$ and $B$ with respect to hydroxylation, and by the statistical effect of reaction sites on B relative to A. Hamilton $(17,18)$ has shown the former to be relatively unimportant. Therefore, for the case of anisole, on a purely statistical basis, we expect $k_{2} / k_{1}=4 / 5$. Consequently, the maximum theoretical yield must be less than $100 \%$ because $k_{2}$ does not equal zero.

\section{B. The Cyclodextrin Effect}

1. Cyclodextrin Inclusion Complexes.

Cyclodextrins (also known as cycloamyloses or schardinger dextrins) are ring compounds of $\alpha-1,4-1$ inked D-glucose polymers containing six or more glucose residues; they are formed by action of Bacillus macerans on starch. Table XIX lists some properties of the three cyclodextrins; French (97) and Stewart (98) have reviewed their production, purification, and chemistry. 
Table XIX

Some Properties of the Cyclodextrins

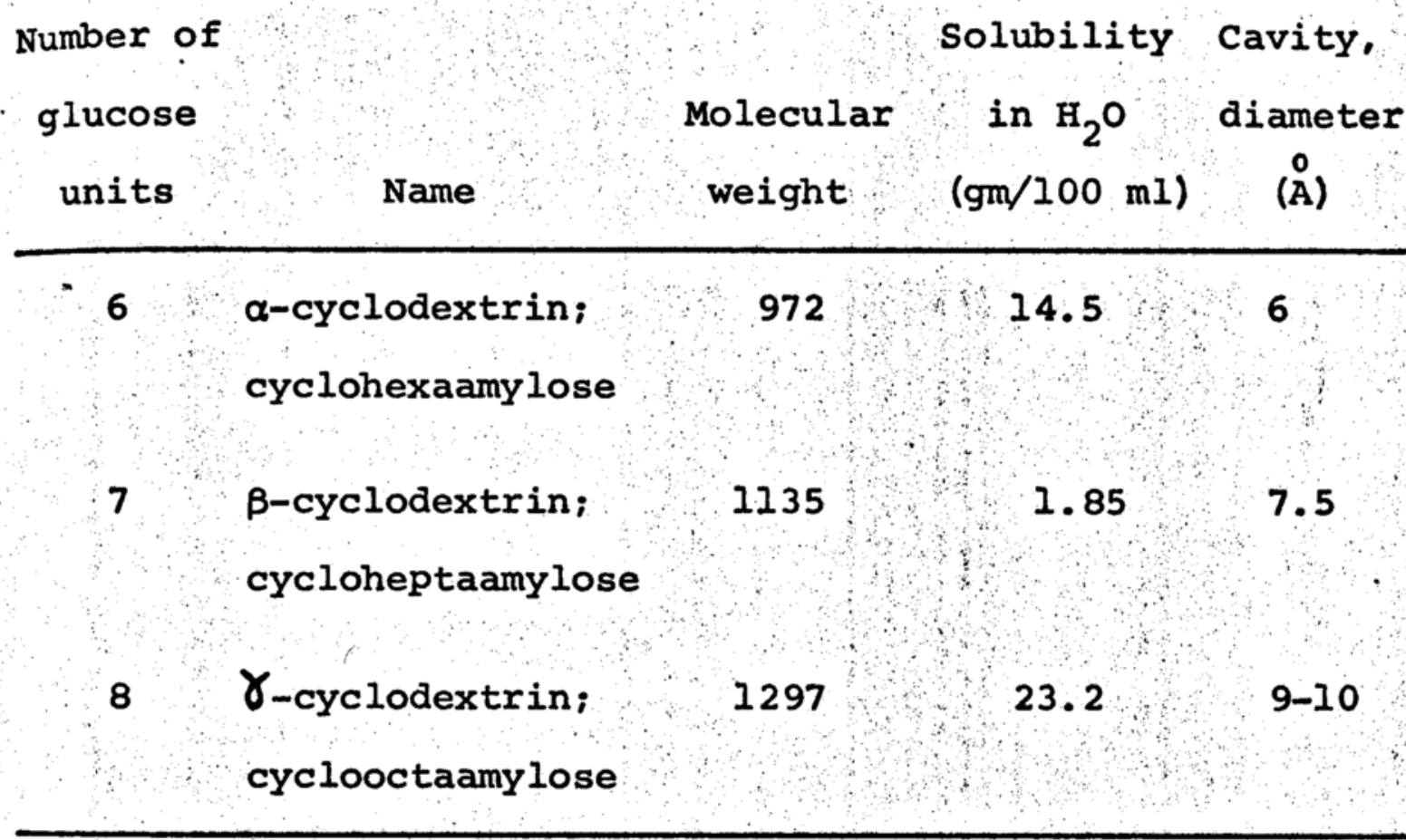


X-ray crystallography $(97,99)$ has established the doughnut shape of the molecules and molecular models have shown that the cavity is longitudinally non-uniform (primary hydroxyl groups reside on one side of the torus whereas secondary - $\mathrm{CHOH}$ groups edge the other rim), containing uniformly spaced bridging acetal oxygen atoms. Based upon energy considerations, it is generally believed that the cavity contains water molecules hydrogen-bonded to the interior oxygen atoms (the cavity is therefore more hydrophilic than hydrophobic).

The hollow structure allows any molecule smaller than its internal diameter to enter and undergo non-covalent interaction with the atoms lining and rimming the cavity; the resulting inclusion complex owes its stability to van der Waals' and dispersion forces. Stoichiometries of $1: 1$ are common although other ratios have been observed.

The ability of cyclodextrins to form a protective shield around the guest molecule and hold it in a certain conformation differentiates this complex from the more common molecular complexes. The stability of the resulting inclusion complex varies, of course, with the size of the guest molecule, but the cavity dimensions for $\alpha$-cyclodextrin are such that most mono- and di-substituted benzene derivatives can easily fit in, while poly-substituted aromatics may be excluded. Table Xx, from cramer (100) lists dissociation constants for several aromatic- $\alpha$-cyclodextrin complexes. It is apparent that a certain selectivity of the association 
Table $\mathrm{xx}$

Dissociation Constants for $\alpha$-Cyclodextrin Complexes (Cramer ${ }^{100}$ ). a

Guest Molecule $\mathrm{b}^{\mathrm{b}}$ $10^{3} \times(\underline{M})^{c}$<smiles>[R]N=Nc1ccc(OC)c([N+](=O)[O-])c1</smiles>

2.6

0.27

2.4<smiles>[R]N=Nc1ccc(O)c(CC)c1</smiles>

2.2<smiles>[R]N=Nc1cc(C)c(O)c(C)c1</smiles>

(no inclusion)

a

In aqueous phosphate buffer at $14^{\circ}$.

$\mathrm{b}$<smiles>[R]O[As]c1ccc(C)c2ccccc12</smiles>

C Determined spectrophotometrically. 
process is occurring, as revealed with the phenylazo-1naphthalene-4-sulfonates. 5 The introduction of one additional methyl group into the aromatic ring (in the case of the 3 ', 5'-dimethyl-4-hydrophenylazo compound), results in its complete exclusion. It should be noted that stability constants for complex formation $(I / K)$, on the order of $10^{3}$, are quite large.

Cycloamyloses have been suggested as enzyme models because of the geometrical control of inclusion complexes noted above. Furthermore, they have been shown to impose both rate accelerations and decelerations on organic reactions (101), such as the acceleration of diphenylpyrophosphate cleavage (102) and the deceleration of the hydrolysis of benzoic acid esters (103). Bender and co-workers (104, 105) have shown a close correspondence between the cyclodextrin-catalyzed hydrolysis of esters and that catalyzed by chymotrypsin. Many characteristics of the enzyme-catalyzed reaction, including saturation, competitive inhibition, and non-productive binding, were exhibited by cyclodextrin.

Breslow $(106,107)$ reported that, in the presence of both $\alpha$ - and $\beta$-cyclodextrins, anisole was selectively chlorinated by HOCl. With cyclohexaamylose, ortho-chlorination was completely suppressed while a specific catalytic effect was

5 The cyclodextrin is presumably "threaded" over the phenylazo portion of the guest molecule, with the naphthyl group protruding $(100)$. 
observed for para-chlorination. In the complex of anisole with $\beta$-cyclodextrin (cycloheptaamylose), ㅇ-chlorination was also blocked, but p-chlorination proceeded at the same rate as in free solution. It is believed that chlorination occurs via conversion of one of the cyclodextrin - $\mathrm{OH}$ groups to $-\mathrm{OCl}$ upon reaction with $\mathrm{HOCl}$; the electrophilic chlorine is then delivered to the para position of an anisole molecule bound in the cavity. Within the complex, the ortho position is inert toward chlorination, and the para position is more reactive than it is in the unbound molecule. Specific catalytic effects by $a$-cyclodextrin have been noted with the hydrolysis of phenyl esters (101) as well.

Cramer studied an imidazolyl modified cyclodextrin (108) for its catalytic activity and observed a modest rate increase for the hydrolysis of p-nitrophenyl acetate. A nickel chelate of pyridine carboxaldoxime and an appropriately acylated cyclodextrin (109) was similarly investigated by Breslow.

2. Hypothesis for Stabilization by Cyclodextrin. The problem of instability of the initial product of hydroxylation (due to its subsequent reaction), which rendered the Hamilton system ineffective as an analytical reagent, was overcome by incorporating a cyclodextrin into the reaction mixture (cf. Figure 7). The hypothesis was formulated that in the presence of cyclodextrins inclusion complexes of aromatic compounds will be formed, and that 
selectivity may be achieved on both an equilibrium basis (different extents of complexation by the aromatic substrate and the phenolic product) and a kinetic basis (inaccessibility of sites for further hydroxylation of a complexed phenol). By binding the aromatic compound within the "protective sheath" of the cyclodextrin, the apparent rate constants should be altered, presumably decreasing the $B \rightarrow C$ rate relative to $A \rightarrow B$. Figure 7 shows that the expected stabilizing effect was observed. At least part of the stabilizing effect is probably due to concurrent decomposition of the Hamilton system (the aging phenomenon has already been discussed), which alters the catalytic effectiveness of the enediol and concomitantly uses up peroxide. Presumably, cyclodextrin merely delays the attainment of the maximal initial product concentration long enough for the incursion of the subsequent hydroxylation to be prevented by degradation of the reagent. The extent to which the binding phenomenon invoked in the hypothesis is responsible for the stabilization has not been established; certainly some binding must occur in these systems, but the later experiments with glucose indicate that it may play a minor role in the stabilizing effect. This work is an interesting example of a useful experimental design based upon a (possibly) faulty hypothesis.

Examination of Figure 7 reveals the similarity in effects of adding cyclodextrin or decreasing peroxide. This suggests that cyclodextrin may function, in part, by removing 
the peroxide (or the hydroxylating agent) ${ }^{6}$ from availability by a "general stabilization mechanism" to be discussed shortly. This would account for (I) the slower rate of A $\rightarrow$ B reaction, (2) the constant final yield, (3) the decreased yield as cyclodextrin concentration is increased; because all three effects were produced by simply decreasing peroxide. 6 However, because inclusion complex formation occurs readily between aromatics and cyclodextrin (the stability constants are large; $\mathrm{K} \sim 10^{3}$ ), the mechanism for stabilization by cyclodextrin must be more complex than that for the "general" stabilizers (eg., glucose, lactose). Experimental evidence for these mechanistic differences is provided by way of contrasting behavior for phenobarbital and chlorpheniramine in a cyclodextrin-modified, and a glucose-modified Hamilton system (see Figures 17 and 18 respectively).

From the mechanistic viewpoint a knowledge of the distribution and absolute yields of monohydroxylated products in the presence and absence of cyclodextrins is essential. Without this information, it would be impossible to determine whether selective hydroxylation is occurring within the dextrin cavity or whether only free substrate is being hydroxylated. No attempts were made, however, to obtain these data

6

Decreasing peroxide concentration would concomitantly reduce the concentration of hydroxylating agent. If the hydroxylating agent were the species that were destroyed, then any effect resulting from its decreased concentration would mimic that which would occur if peroxide were removed. 
because the cyclodextrin-modified system offered no advantages from an analytical point of view over the glucose system. In fact the latter appears to be simpler (no inclusioncomplex formation is possible), cheaper (glucose is readily available in pure form), and more rapid (reactions can be run at higher temperatures).

\section{c. General Stabilization Effects}

1. Kinetic Schemes.

The suggestion that cyclodextrins may function, in part, by decreasing the availability of the hydroxylating agent, led to the investigation of other compounds for their stabilizing ability. As previously noted, the effect was quite general, occurring with other "reducing sugars" as well as with a number of aliphatic compounds. Hamilton (18), in fact, observed that most common water-soluble organic solvents at low concentrations markedly inhibit the rate of anisole hydroxylation, leading him to speculate that the organic solvent must intercept the oxidizing intermediate in the catalytic reaction.

Eq. (21) represented an attempt to describe the Hamilton system in terms of a simple $\mathrm{A} \rightarrow \mathrm{B} \rightarrow \mathrm{C}$ sequence. The system is, however, much more complex because of the "aging" phenomenon noted above. Moreover, catechol can be viewed as an "inhibitor" as well as a catalyst because of the dependence of yield on its initial concentration (cf. Table VII). 
Consequently, the kinetic scheme should, at the very least, be modified to incorporate the "inhibitor concept", which would provide an additional pathway for the loss of oxidizing agent. This idea is shown in Eq. (23),

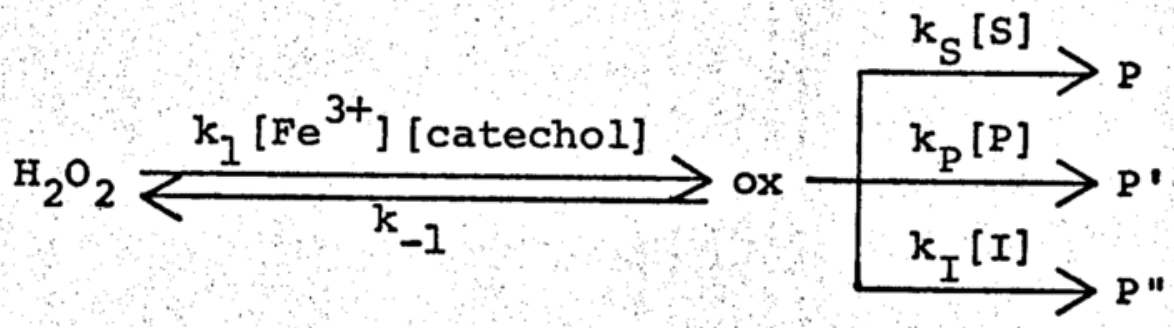

where ox denotes the hydroxylating species; $s$, the aromatic substrate (formerly referred to as A); $P$, the initial product of hydroxylation (formerly referred to as $B^{\prime}$; $P^{\prime}$, subsequent dihydroxylated products derived from $P$ (referred to earlier as C); I, the inhibitor whose identity can remain unspecified $^{7}$; P", a product of unspecified nature derived from the inhibitor; $k_{S}, k_{P}$, and $k_{I}$ are rate constants which refer to their respective substrates.

Then:

$$
\frac{d[P]}{d t}=k_{S}[S][O x]-k_{P}[P][o x]
$$

and

$$
\begin{aligned}
& \frac{d[o x]}{d t}=k^{\prime}\left[\mathrm{H}_{2} \mathrm{O}_{2}\right]-k_{-I}[o x]-k_{S}[S][o x]- \\
& k_{P}[P][o x]-k_{I}[I][o x]
\end{aligned}
$$

7

The inhibitor may be catechol and buffer in the unmodified system and/or any stabilizer incorporated into the Hamilton system. 
where $k^{\prime}=k_{1}\left[\mathrm{Fe}^{3+}\right][$ catechol]. Applying the steady-state condition to Eq. (25) gives

$$
[\mathrm{ox}]_{S S}=\frac{k^{\prime}\left[\mathrm{H}_{2} \mathrm{O}_{2}\right]}{k_{-1}+k_{S}[\mathrm{~S}]+k_{P}[\mathrm{P}]+k_{I}[\mathrm{I}]}
$$

The material balance equations are

$$
\begin{aligned}
& {[\mathrm{S}]_{0}=[\mathrm{S}]+[\mathrm{P}]+\left[\mathrm{P}^{\prime}\right]} \\
& {\left[\mathrm{H}_{2} \mathrm{O}_{2}\right]_{0}=\left[\mathrm{H}_{2} \mathrm{O}_{2}\right]+[\mathrm{OX}]+[\mathrm{P}]+2\left[\mathrm{P}^{\prime}\right]+} \\
& \mathrm{n}\left[\mathrm{I}_{0}-\mathrm{I}\right]
\end{aligned}
$$

We assume $n=I$ and adjust $[I]_{0}$ accordingly, i.e.. the inhibitor concentration is given in normality rather than molarity.

$$
\text { Define: } \begin{aligned}
r_{0} & =\frac{\left[\mathrm{H}_{2} \mathrm{O}_{2}\right]_{0}}{[\mathrm{~S}]_{0}} \\
r_{t} & =\frac{\left[\mathrm{H}_{2} \mathrm{O}_{2}\right]}{[\mathrm{S}]} \\
\alpha & =\frac{[\mathrm{S}]}{[\mathrm{S}]_{0}} \\
y & =\frac{[\mathrm{P}]}{[\mathrm{S}]_{0}} \\
x & =\frac{[\mathrm{I}]_{0}}{[\mathrm{~S}]_{0}} \\
z & =\frac{[\mathrm{I}]}{[\mathrm{S}]_{0}} \\
&
\end{aligned}
$$


where $r_{0}$ and $r_{t}$ represent the ratios of peroxide to substrate at time $t=0$ and any time $t$ respectively; $\alpha$, the fraction unreacted of substrate; and $y$, the fractional yield of the initial hydroxylation product. Then:

$$
r_{0}=\frac{\left[\mathrm{H}_{2} \mathrm{O}_{2}\right]+[\mathrm{OX}]+[\mathrm{P}]+2[\mathrm{P} I]+\left[\mathrm{I}_{0}-\mathrm{I}\right]}{[\mathrm{S}]_{0}}
$$

Assuming [ox] $\ll\left[\mathrm{H}_{2} \mathrm{O}_{2}\right]$ and using Eq. (27) gives

$$
r_{0}=\alpha r_{t}+2-2 \alpha-y+x-z
$$

Then, combining Eqs. (24), (26) and the above definitions,

$$
\frac{d[P]}{d t}=\left[\alpha k_{S}-y k_{P}\right]\left[\frac{\alpha r_{t} k^{\prime}[S]_{0}}{\frac{k_{-1}}{[S]_{0}}+\alpha k_{S}+y k_{P}+z k_{I}}\right]
$$

The conditions for a stable state $(\mathrm{d}[\mathrm{P}] / \mathrm{dt}=0)$ are

$$
r_{t}=0
$$

and/or

$$
\alpha \mathrm{k}_{\mathrm{S}}-\mathrm{y \textrm {k } _ { \mathrm { p } }}=0
$$

Applying condition (38) to Eq. (36) gives the stable-state yield:

$$
y=2(1-\alpha)+x-z-r_{0}
$$

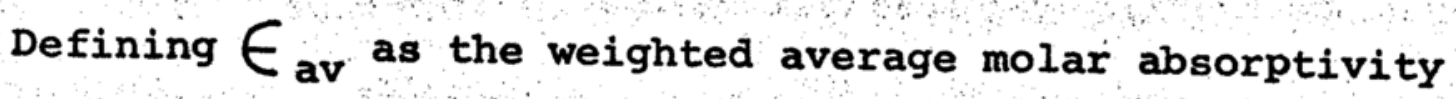


of the coupled monohydroxy products, we have

$$
A=\epsilon_{a v} b[P]
$$

and since $\mathrm{Y}=[\mathrm{P}] /[\mathrm{S}]_{0}$.

$$
A=\epsilon_{a v}{ }^{b y}[s] 0
$$

Substituting Eq. (40) into Eq. (42) gives

$$
A=\epsilon_{a v}^{b}[S]_{0}\left[2(1-\alpha)+x-z-r_{0}\right]
$$

By making the rough approximation that all rate constants are the same, i.e., $k_{S}=k_{P}=k_{I}$, we get the further simplification

$$
[I] /[I]_{0}=[S] /[S]_{0}
$$

or

$$
\alpha \mathbf{x}=\mathbf{z}
$$

Thus

$$
A=\epsilon_{a v}^{b}[s]_{0}\left[(2+x)(1-\alpha)-r_{0}\right]
$$

Eq. (46) relates the absorbance to initial substrate concentration $\left[\mathrm{S}_{0}\right]$, and yield $\mathrm{y}$.

In the absence of quantitative data on species distribution at the conclusion of the reaction, little can be said about the dependence of $\alpha$ on [S] 0 . The quantities $x$ and $r_{0}$. however, are initial conditions, and it is interesting to 
analyze their effects on the appearance of Beer's law plots developed under different initial conditions. The quantity $\left(x-r_{0}\right)$ (see Eq. 43) can be expanded to give Eq. (47).

$$
x-r_{0}=\frac{[\mathrm{I}]_{0}-\left[\mathrm{H}_{2} \mathrm{O}_{2}\right]_{0}}{[\mathrm{~S}]_{0}}
$$

Within a given Beer's law study, both $[\mathrm{I}]_{0}$ and $\left[\mathrm{H}_{2} \mathrm{O}_{2}\right]_{0}$ are usually constant, and $[\mathrm{S}]_{0}$ changes. Let

$$
c=[\mathrm{I}]_{0}-\left[\mathrm{H}_{2} \mathrm{O}_{2}\right]_{0}
$$

where $c$ is a constant. Then Eq. (47) may be differentiated with respect to $[\mathrm{S}]_{0}$ to obtain

$$
\frac{d\left[x-r_{0}\right]}{d[S]_{0}}=\frac{-c}{[S]_{0}^{2}}
$$

When $x=r_{0}, c=0$ and $d\left[x-r_{0}\right] / d[s]_{0}=0$. Then no change in yield with change in [S] 0 will occur (with respect to the influences of $x$ and $r_{0}$ ). If variations in $\alpha$ are small over the same range of $\left[\mathrm{S}_{0}\right.$, a linear Beer's law plot will be observed.

Table XXI lists the range of $\mathrm{x}$ for several stabilizers on both a molar basis and a normal basis. The difficulty, of course, is relating the two concentration scales, because the stoichiometries are unknown. However, there appears to be an overlap between the ranges within which $x$ and $r_{0}$ vary. Thus, the condition $x \approx r_{0}$ may be the essential feature for 
Table XXI

Comparison of $\mathbf{x}$ Values for Several stabilizers

in a Modified Hamilton System

\begin{tabular}{llllll}
\hline & ${ }^{[I]_{0}^{b}}$ & Range of $x^{c}$ & & Range of $\times$ \\
Stabilizer & $\underline{(M)}$ & (molar) & $n^{d}$ & (normal) \\
\hline Glucose & $4 \times 10^{-3}$ & $4-40$ & 6 & $24-240$ \\
Sorbitol & $4 \times 10^{-3}$ & $4-40$ & 6 & $24-240$ \\
Lactose & $3 \times 10^{-3}$ & $3-30$ & 12 & $36-360$ \\
Acetonitrile & $2 \times 10^{-1}$ & $200-2000$ & 1 & $200-2000$ \\
Cyclodextrin & $1 \times 10^{-3}$ & $1-10$ & 42 & $42-420$ \\
\hline
\end{tabular}

a $\left[\mathrm{H}_{2} \mathrm{O}_{2}\right]_{0}=4 \times 10^{-3} \underline{\mathrm{M}} ;[\mathrm{S}]_{0}=1 \times 10^{-3} \underline{\mathrm{M}}-1 \times 10^{-4} \underline{\mathrm{M}}$; therefore $r_{0}=4-40$.

b Median value of stabilizer concentration giving maximal yields.

$\mathbf{x}=[\mathrm{I}]_{\alpha}[\mathrm{S}]_{0}$.

$\mathrm{d}_{\mathrm{n}}=$ number of oxidizable carbons. 
the obtention of a linear Beer's law plot. 8

There were two conditions under which linear Beer's law plots were observed, namely,

(a) when $r_{0}$ is held constant at 2 or less and no external stabilizer is added. It is possible that, under these conditions, $x$ is also small but non-zero, the role of $I$ being played by catechol, acetate buffer and product $\mathrm{P}^{\prime}$.

(b) when $r_{0}$ is large and variable and $x$ is similarly large and variable. This represents the typical analytical situation in the presence of "stabilizer".

When $x<r_{0}, c$ is negative and $d\left[x-r_{0}\right] / d[s]_{0}$ is positive. This corresponds to positive curvature in a Beer's law plot because as $[S]_{0}$ increases, the yield increases. This condition appears to conform to the case of large $r_{0}$ values with no added stabilizer (cf. Figure 16).

When $x>r_{0}, c$ is positive and $d\left[x-r_{0}\right] / d[s]_{0}$ is negative. Negative curvature can be seen in the Beer's law plot. The two conditions under which this effect is observed are,

(a) when $r_{0} \leq 2$ and $\left[\mathrm{H}_{2} \mathrm{O}_{2}\right]_{0}$ is constant. If $x=r_{0}$ at the lowest value of [S] $]_{0}$ thus giving an initial linear Beer's law plot, as [S] $]_{0}$ increases, $x$ becomes larger than $r_{0}$ if product $P$ ' contributes to the stabilizing action by competing for the oxidizing agent.

(b) when $r_{0}>2$, but $x>r_{0}$ for the same reason given under (a).

8 The contribution of $\alpha$ to yield could dampen the dependence on $x$ and $r_{0}$. 
The other possible condition for stability is given by Eq. (39), namely, when $\alpha k_{S}=y k_{P} \frac{d[P]}{d t}=0$. Applying this condition to Eq. (36) and assuming $k_{S}=k_{P}=k_{I}$ (and Eq. 45) gives

$$
y=\frac{\left(r_{0}-2\right)-x}{\left(r_{t}-3\right)-x}
$$

Eq. (50) predicts that as $[\mathrm{s}]_{0} \rightarrow \infty, r_{0} \rightarrow 0, r_{t} \rightarrow 0$, $x \rightarrow 0$ and $y \rightarrow 2 / 3$. Chemically, this means that as [s] increases with these reasonable rate constant equivalencies, a stable-state with dynamic production and loss of $P$ will result with the maximum possible yield of $2 / 3$, this without depletion of peroxide. In effect, for each 3 moles of $S$ converted to $\mathrm{P}, 1$ mole of $\mathrm{P}$ is converted to $\mathrm{P}^{\prime}$. Experimental evidence for a maximum yield of about $2 / 3$ of $100 \%$ is, incidentally, provided in Figure 25 which shows a plot of (absorbance $^{-1}$ vs (concentration) ${ }^{-1}$ for the hydroxylation of anisole and nitrobenzene. It is apparent that as [S] $\rightarrow_{0} \infty$, $[\mathrm{s}]_{0}^{-1} \rightarrow 0$ and the intercepts give absorbances whose yields correspond to about $2 / 3$.

Eq. (50) also predicts stability at values of $r_{0}>2$ when an inhibitor (stabilizer) is present. This equation may be substituted into Eq. (42) to obtain an expression relating absorbance to initial substrate concentration and yield, but the outcome is an equation more complicated than Eq. (46). Moreover, when $r_{t}=0$ (which, incidentally, must ordinarily be the stable-state condition, as evidenced by the 
Figure 25: Double-reciprocal plot of (absorbance) ${ }^{-1}$ vs (concentration) $^{-1}$ for hydroxylation of anisole and nitrobenzene in an unmodified Hamilton system at $25^{\circ}, 1.18 \times 10^{-4}$ M catechol, $5.88 \times 10^{-5} \underline{\mathrm{M}} \mathrm{Fe}(\mathrm{III}), \mathrm{pH} 4.0,2 \mathrm{hr}$ reaction time.

O. Anisole; $8.2 \times 10^{-4} \mathrm{M} \mathrm{H}_{2} \mathrm{O}_{2}$

O. Nitrobenzene; $9.1 \times 10^{-4} \mathrm{M} \mathrm{H}_{2} \mathrm{O}_{2}$ 65\% yield for anisole ${ }^{\mathrm{a}}$ based upon a standard of o- and $\mathrm{p}$-methoxypheno ${ }^{\mathrm{b}}$ in a ratio of $2: 1$; 60\% yield for nitrobenzene ${ }^{a}$ based upon a standard composed of $\mathrm{O}_{-}, \mathrm{m}-$, and $\mathrm{p}$-nitrophenol ${ }^{c}$ in a ratio of $2: 1: 1$.

a Yields are determined by extrapolating $\frac{1}{[S]_{0}}$
to zero and are based upon initial peroxide concentration. This is reasonable because as $[S]_{0} \rightarrow \infty$, all available hydroxylating agent should react with substrate.

b $5.23 \times 10^{-4}$ M total methoxyphenol; $A_{500}=$ $4.04 \times 10^{-4} \mathrm{M}$ total nitrophenol; $\mathrm{A}_{405}=$
0.856 (nitrophenolate 


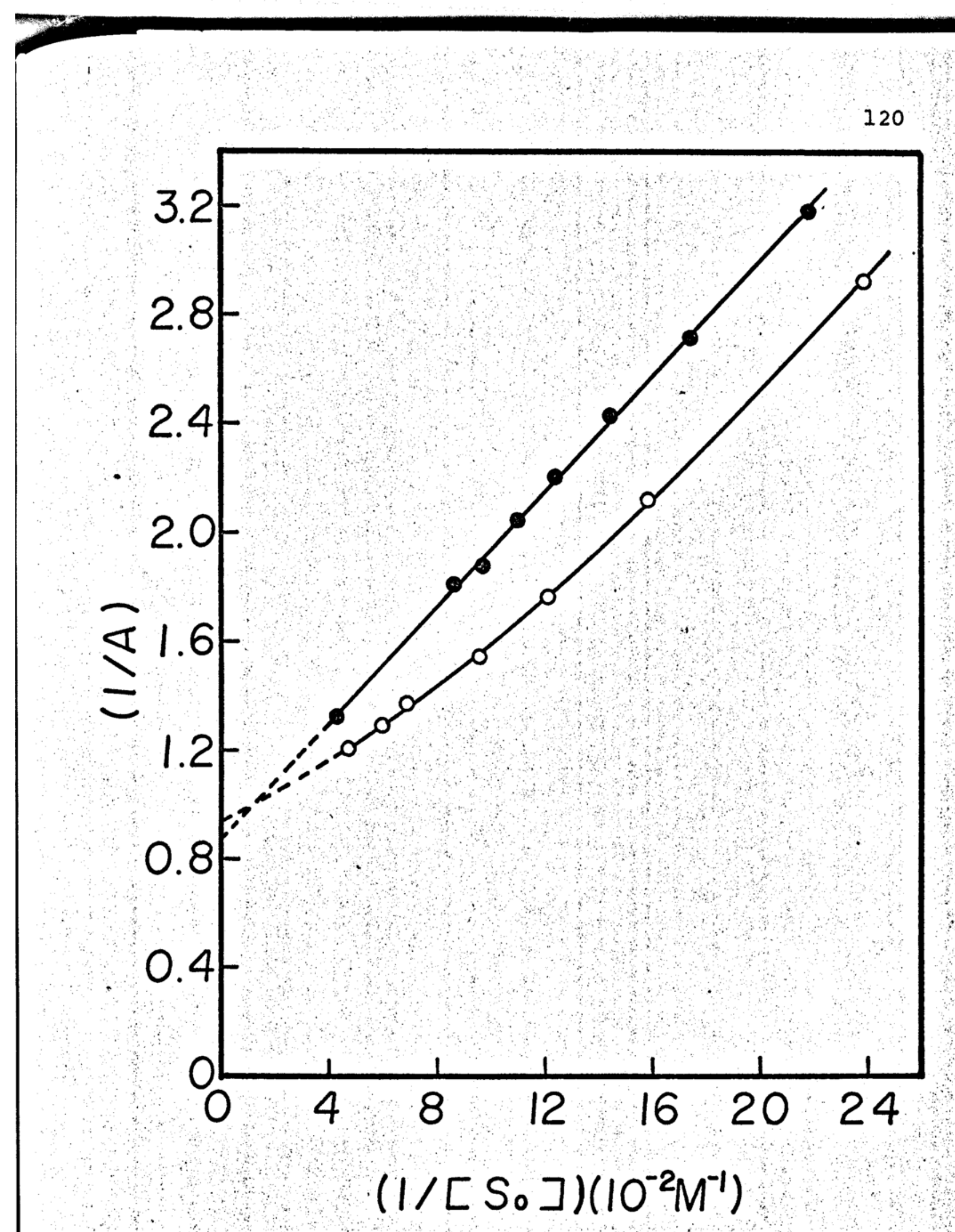


loss of $P$ upon addition of more Hamilton reagent, Figure 26), stability will be achieved whether or not $\alpha k_{S}=y k_{p}$.

\section{Comparison of stabilizers.}

The material balance equation, Eq. (28), contains a term $n\left([I]_{0}-[I]\right)$ for the stabilizer I. By assigning a value to $n$, its concentration is specified in normality rather than molarity. The relationship between these concentration units is probably not simple because the hydroxylating agent is so non-specific that it apparently oxidizes aldehydes to ketones and alcohols to carboxylic acids as readily as it hydroxylates an aromatic ring (19). However, based upon the data in Table XVI and related discussion, a qualitative relationship seems to exist between the number of oxidizable carbons of the inhibitor, and its optimal concentration range. Thus, the stabilizers fall in this order of decreasing "effectiveness" as measured by the molar concentration required for optimal results: cyclodextrin > lactose > glucose $\sim$ sorbitol $>$ dioxane > isopropyl alcohol > acetone > acetonitrile.

\section{Analysis by Hydroxylation}

\section{Proposed Method.}

The proposed analytical method for the determination of aqueous solutions of aromatic compounds can be found in the Experimental section on page 29. Although at $75^{\circ}$ reactions appear to be over in about $5 \mathrm{~min}$, a $10 \mathrm{~min}$ reaction time was arbitrarily chosen. 


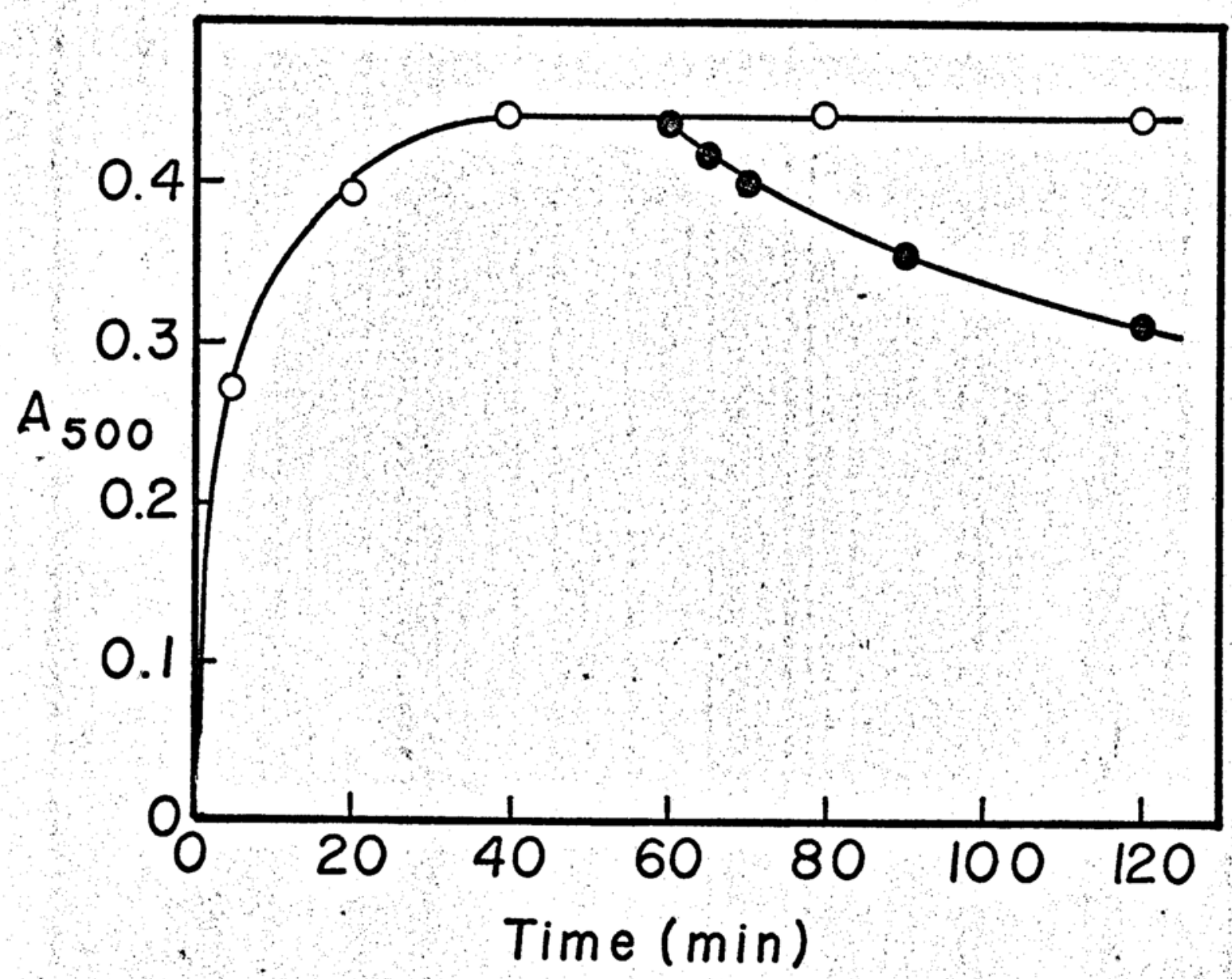

Figure 26: Effect of addition of Hamilton reagent at the stable state $(d[P] / d t=0)$ on the time course for anisole hydroxylation in a cyclodextrinmodified Hamilton system; $1.16 \times 10^{-4} \mathrm{M}$ catechol, $5.94 \times 10^{-5} \mathrm{M} \mathrm{Fe}(\mathrm{III}), 3.55 \times 10^{-3} \mathrm{M} \mathrm{H}_{2} \mathrm{O}_{2}$, $6.37 \times 10^{-4} \underline{\mathrm{M}}$ anisole, $1.08 \times 10^{-3} \underline{\mathrm{M}}$ cyclodextrin, $2 \mathrm{hr}$ reaction time, $25^{\circ}, 4$-AAP finish.

O. Normal time course.

O. Hamilton reagents (Fe (III), catechol, and $\mathrm{H}_{2} \mathrm{O}_{2}$ ) added at stable-state. 
2. Range of Applicability.

The analytical method is applicable to aromatic compounds in the range $10^{-4}$ M to $10^{-3}$ ㅆ provided that an aqueous solution can be prepared to contain at least 2.0 umoles of sample in $20 \mathrm{ml}$ of water ${ }^{9}$, and that the corresponding phenol responds to the 4-AAP finish. Below $10^{-4} \underline{\mathrm{M}}$, the final analytical signal may be too low; above $10^{-3} \underline{M}$, negative curvature may occur due to an insufficient concentration of the hydroxylating agent in solution.

\section{The Finish.}

The 4-aminoantipyrine finish is both general and sensitive for the determination of aqueous phenols $(32,34-39)$. Moreover, the method responds in the presence of components of the Hamilton system and offers the additional advantage of specificity for mono-hydroxyphenols. Consequently, the enediol catalyst does not interfere with the determination of the product phenol from the hydroxylation reaction. The borate buffer acts both to quench the hydroxylation reaction and to serve as medium for color development simplifying the analytical procedure.

Fading of the color has been associated with the presence of electron-withdrawing groups on the aromatic ring (cf. Figure 24). Furthermore, if a substituent were to be oxidized

9 The $\mathrm{pH}$ of this solution may be easily adjusted to the range 3.5 to 4.0 . The remaining $5 \mathrm{ml}$ could be used for reagent, stabilizer, and a more concentrated buffer. 
to an electron-witharawing group by the Hamilton system, then the resulting phenol would be expected to exhibit color fading. For example, mandelic acid, ephedrine, phenylalanine, and sulfanilamide all showed loss of color with the 4-AAP method even though their substituents are not electronwithdrawing. Furthermore, the amine substituent of sulfanilamide apparently was oxidized to a nitro-group because the compound showed the characteristic nitrophenolate absorption after treatment with the Hamilton system.

The quinoidal structure of a 4-AAP-coupled dye is given below, where $x$ and $y$ are substituents on the parent phenol. It is apparent that substituent $\mathbf{x}$ (ortho to the original

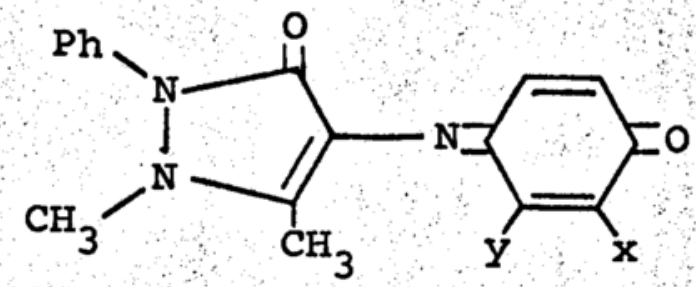

phenolic function) is in direct conjugation with the quinoidal antipyrine ring, while substituent y (ortho to the antipyrine nitrogen) is directly conjugated to the phenolic oxygen. 10 A possible route to decomposition of the coupled antipyrine dye may involve hydrolytic cleavage of the antipyrine ring under the influence of electron-withdrawal. The o-substituted phenols decompose faster (i.e., their coupled products

10

The quinoid structure is essential for transmission of electronic effects by resonance delocalization from the meta position relative to the coupled antipyrine. 
decompose) than their m-substituted counterparts because of the greater resonance delocalization effect on the 5-membered ring.

4. Comments on Product Yield and Distribution. The apparent molar absorptivity, $\epsilon_{\text {app }}$ is defined as the absorbance of the coupled phenol product (with 4-aminoantipyrine) at its maximum wavelength divided by the total sample concentration (regardless of fate) in the final colorimetric solution. The magnitude of $\epsilon_{\text {app }}$ (see Tables $x, x I I$, and $\mathrm{XV}$ ) reflects the yield of the initial hydroxylation product, the isomer distribution, and the molar absorptivities of the coupling products. Under conditions of the color development, phenol itself gives a molar absorptivity of $1.23 \times 10^{4}$; thus the yield of phenol from benzene can be unambiguously calculated using the apparent molar absorptivity of benzene given in Table $\mathrm{x}$.

$$
\% \text { Yield }=\frac{4.5 \times 10^{3}}{1.23 \times 10^{4}} \times 100=37 \%
$$

Yields from other substrates are probably similar.

Eq. (5I) gives the yield of phenol from benzene in a cyclodextrin-modified system at $25^{\circ}$. If the value of $2.9 \mathrm{x}$ $10^{3}$ were used from Table $\mathrm{XV}$ for a glucose-modified system at $75^{\circ}$, then the yield would be about $24 \%$. The disparity may be related to a temperature effect as well as to a different mechanism of stabilization for glucose. The concentration of stabilizer may also be involved since depressed yields 
are observed when the optimal stabilizer concentration range is exceeded.

A better comparison between the two modified Hamilton systems can be made by determining the yield of phenol from benzene for both systems at $25^{\circ}$ at corresponding inhibitor concentration. One obtains a $40 \%$ yield with cyclodextrin and a $35 \%$ yield with glucose ${ }^{l l}$, indicating only a small inhibitor contribution to yield differences.

Table XXII compares molar absorptivities using a $25^{\circ}$ cyclodextrin-modified, and a $75^{\circ}$ glucose-modified Hamilton system. As noted above, differences in apparent molar absorptivity are related to the temperature effect, the stabilizer concentration, and perhaps a mechanistic difference. However, an average molar absorptivity of $(2.8 \pm 1.1) \times 10^{3}$ obtained for the cyclodextrin system, and $(2.7 \pm 1.3) \times 10^{3}$ obtained for the glucose system, indicates that no significant distinction between the two systems exists.

\section{Mixtures and Interferences.}

Due to the non-specificity of the hydroxylating agent, it would be difficult to determine mixtures of aromatics. Under conditions of stability where the effective concentration of oxidizing agent has been "competitively" reduced,

$111.17 \times 10^{-4} \underline{\mathrm{M}}$ catechol, $5.95 \times 10^{-5} \underline{\mathrm{M}} \mathrm{Fe}(\mathrm{III}), 4 \times 10^{-3} \underline{\mathrm{M}}$ $\mathrm{H}_{2} \mathrm{O}_{2} ; 1.07 \times 10^{-2} \underline{M}$ glucose, $120 \mathrm{~min}$ reaction time; $1.20 \times$ $10^{-3} \underline{\mathrm{M}}$ cyclodextrin, $60 \mathrm{~min}$ reaction time. 
Table XxII

Comparison of Apparent Molar Absorptivities for Aromatic Compounds in Modified Hamilton Systems.

\begin{tabular}{|c|c|c|}
\hline \multirow{2}{*}{ Sample } & \multicolumn{2}{|c|}{$10^{-3} \in$ app } \\
\hline & $\begin{array}{c}\text { Cyclodextrin }{ }^{a} \\
\left(25^{\circ}\right)\end{array}$ & $\begin{array}{c}\text { Glucose }^{b} \\
\left(75^{\circ}\right)\end{array}$ \\
\hline Benzene & 4.5 & 2.9 \\
\hline Anisole & 3.9 & 4.4 \\
\hline Acetanilid & 3.9 & 3.4 \\
\hline Phenacetin & 2.7 & 3.1 \\
\hline Benzoic acid & 2.5 & 1.7 \\
\hline o-Methoxybenzoic acid & 3.6 & 3.7 \\
\hline m-Methoxybenzoic acid & 2.4 & 2.8 \\
\hline p-Methoxybenzoic acid & 4.2 & 5.5 \\
\hline Mandelic acid & 2.1 & 1.3 \\
\hline Atropine & 2.6 & 2.1 \\
\hline Ephedrine & 2.7 & 2.2 \\
\hline Phenobarbital & 1.1 & 1.3 \\
\hline Chloramphenicol & 0.7 & 0.8 \\
\hline
\end{tabular}
a Data from Table $\mathrm{x}$.
b Data from Table XV. 
the presence of an additional aromatic sample would only reduce the yield of both because there just is not enough hydroxylating agent. The same argument would apply to the determination of aromatics in the presence of aliphatics. However, no interference would be observed provided that the total concentration of inhibitor (aliphatic), regardless of ițs source, remained within the maximal "stabilizer" range. Thus, more acetonitrile could be tolerated than, say, dioxane, because of the qualitative relationship noted above, between the number of oxidizable carbons and the concentration needed to produce a desired effect. This would explain why, for the determination of $6 \times 10^{-4} \underline{M}$ acetanilid in a cyclodextrin-modified Hamilton system, $10^{-3} \underline{M}$ dioxane caused a $40 \%$ decrease in response. Apparently, the combination of cyclodextrin and dioxane exceeded the "stabilizer range" and the yield dropped. Here, it could be argued that cyclodextrin contributed to the lower yield (it acted as an inhibitor or interferent), because by merely reducing its concentration, the yield could be returned to its maximal value.

similarly, the independence of yield on catechol concentration above $1.2 \times 10^{-4} \underline{M}$ in a stabilizer-modified Hamilton system $^{12}$ (cf. Figure 21) may be because the combination of

12 In an unmodified Hamilton system, a maximal catechol concentration was observed at $1.2 \times 10^{-4} \mathrm{M}$. Above this value (at constant ratio of Fe(III) to catechol), the yield dropped, presumably because of the inhibitory effect of catechol, i.e.. catechol was an interferent depleting the concentration of available oxidizing agent. 
cyclodextrin (or glucose) and excess catechol was still within the maximal stabilizer range.

Separation of the aromatic sample compound from potential interferences (e.g., tablet excipients) prior to hydroxylation would circumvent the inhibitory effect on yield displayed by all interferents. Under conditions where lactose caused a decreased response from phenobarbital hydroxylation, extraction of the aromatic sample into chloroform, evaporation of the chloroform, and subsequent hydroxylation, returned the yield to its maximal value.

Negative intercepts in a working curve of absorbance against concentration represent an additional, though minor, complication. Intercepts were observed for the following sample compounds listed in Table XV: anisole, phenacetin, ephedrine, ㅇ-methoxybenzoic acid and p-methoxybenzoic acid. The intercept values, furthermore, were not reproducible. These results may be related to different extents of hydroxylation of catechol in the blank and sample solutions. As noted earlier, catechol itself should be susceptible to hydroxylation, and the extent of reaction is probably related to the initial ratio of peroxide to substrate. Competition for the hydroxylating agent in the sample solution (between aromatic and catechol) is absent in the blank. Thus, more catechol must react in the blank than in the sample simply because the ratio of peroxide to catechol is different in the two solutions.

The volume of potassium ferricyanide $(0.1 \mathrm{ml}$ or $0.2 \mathrm{ml})$ 
added to the final analytical solution for color development contributes slightly to the reagent background. Since the smaller volume still represents an excess of oxidizing agent, the procedure was modified to reflect this volume change. 


\section{SUMMARY}

The phenyl group as a functional group offers the possibility of a general approach to the detection and determination of aromatic compounds [K. A. Connors and K. S. Albert, Anal. Chem., 44, 879 (1972)]. In this context, aromatic hydroxylation is an attractive reaction because the product, a phenol, is amenable to detection and determination by many methods. Of the several aqueous hydroxylating agents that have been proposed, the Hamilton system, consisting of an aqueous solution of ferric ion, catechol, and hydrogen peroxide, seemed to provide advantages of simplicity, yield, and speed. This thesis reports the further development and application of this system to the quantitative determination of some aromatic compounds.

As an analytical reagent, in which the hydrogen peroxide in the Hamilton system must be in excess over sample compound, hydroxylation continues beyond the introduction of the first hydroxy group. With the quantitative measurement employed here (oxidative coupling with 4-aminoantipyrine), only the monohydroxy compound appears to be detected. By reducing the initial ratio of peroxide to aromatic to 2.0 or less, stable absorbance values were obtained, the yield depending upon this ratio.

The instability of the initial hydroxylation product has been overcome by incorporating a "stabilizer" in the reaction mixture. Two such modified Hamilton systems which 
were studied extensively are those of cyclodextrin at $25^{\circ}$ and glucose at $25^{\circ}$ and $75^{\circ}$.

The cyclodextrin-modified Hamilton system stabilizes the initial hydroxylation product at $25^{\circ}$ over a wide range of ratios of peroxide to substrate. Stability is thought to result, in part, from formation of inclusion complexes, the cyclodextrin delaying the attainment of the maximal initial product concentration long enough for the incursion of the subsequent hydroxylation to be prevented by concurrent decomposition of the Hamilton system. Cyclodextrin may also accelerate the degradation of the hydroxylating agent by competing with the aromatic substrate. Many mono- and disubstituted benzene derivatives give linear absorbance-concentration responses in the range $1-8 \times 10^{-4} \underline{M}$ with apparent molar absorptivities between $1 \times 10^{3}$ and $5 \times 10^{3}$. Reaction times are $1-2$ hours at $25^{\circ}$, and the yields are $\mathrm{pH}$ independent over the range $3.5-4.2$.

Glucose has been shown to stabilize the initial hydroxylation product as effectively as cyclodextrin at $25^{\circ}$, and because its mechanism of stabilization does not involve inclusion complex formation (whose stability constants are temperature dependent), the glucose-modified Hamilton system was investigated at $75^{\circ}$. A stabilization scheme involving competition by glucose for the available hydroxylating agent has been proposed to account for the independence of yield on the initial ratio of peroxide to substrate., A kinetic scheme consistent with available data is presented. 
The glucose-modified Hamilton hydroxylating system has been suggested for the analysis of aromatic compounds at $75^{\circ}$. Calibration curves were linear in the range $10^{-4} \underline{\mathrm{M}}$ to $10^{-3} \underline{\mathrm{M}}$ of aromatic compound, and molar absorptivities of $1-5 \times 10^{3}$ were observed. Reaction times were less than $10 \mathrm{~min}$ and $\mathrm{pH}$ independent from 3.5 to 4.0 . No interference was observed for most aliphatic compounds at $10^{-3} \underline{M}$ and for tablet excipients at normal formulation levels. Fading of the color occurred for compounds containing electron withdrawing groups, but extraction into chloroform stabilized the antipyrine dye, besides increasing the sensitivity of the method. This investigation was designed to provide experimental data to test the potential of aromatic hydroxylation as an analytical reaction for the determination of aromatic compounds in aqueous solution. The results establish that (a) hydroxylation is a useful analytical process; (b) a model enzyme system can be exploited for its analytical utility; and (c) the concept of developing analytical selectivity by means of complex formation with cyclodextrins may be worth further exploitation. As one of the few methods available for the phenyl group as a functional group for analytical purposes, this technique has the advantages and limitations of a general functional group method. 


\section{REFERENCES}

1. H. V. Bergmeyer, editor, "Methods of Enzymatic Analysis", Academic Press, New York, 1963.

2. J. R. Gillette, A. H. Conney, G. J. Cosmides, R. W. Estabrook, J. R. Fouts and G. J. Mannering, editors, "Microsomes and Drug Oxidations", Academic Press, New York, 1969.

3. T. C. Bruice and S. Benkovic, "Bioorganic Mechanisms", Vol. 1, W. A. Benjamin, Inc., New York, 1966.

4. W. P. Jencks, "Catalysis in Chemistry and Enzymology", McGraw-Hill, New York, 1969.

5. M. L. Bender, "Mechanisms of Homogeneous Catalysis from Protons to Proteins", John Wiley and Sons, Inc., New York, 1971.

6. D. I. Metelitsa, Usp. Khim., 40, 1175 (1971).

7. R. O. C. Norman and J. R. L. Smith, "Oxidases and Related Redox Systems, Vol. 1, T. S. King, H. S. Mason and M. Morrison, editors, John Wiley and Sons, Inc., New York, 1965, p. 131.

8. J. D. Loudon, Progr. Org. Chem., 5, 47 (1961).

9. O. C. Dermer and M. T. Edmison, Chem. Rev. . 57, 77 (1957).

10. R. T. Williams, "Homolytic Aromatic Substitution", Pergamon Press, New York, 1960, p. 110.

11. F. Haber and J. Weiss, Proc. Roy. Soc. (London), Al47, 332 (1934).

12. W. T. Dixon and R. O. C. Norman, Proc. Chem. Soc.. $1963,97$.

13. R. L. Smith and R. O. C. Norman, J. Chem. Soc., 1963, 2897.

14. A. I. Brodskii and N. A. Vysotskaya, I. Gen. Chem. U.S.S.R., 32, 2241 (1962).

15. R. O. C. Norman and G. K. Radda, Proc. Chem. Soc.. 1962. 138. 
16. G. A. Hamilton, "Hemes Hemoproteins", B. Chance,

R. W. Estabrook and T. Yonetani, editors, Academic Press, New York, 1966, p. 349.

17. G. A. Hamilton and J. P. Friedman, I. Amer. Chem. Soc. , 85, 1008 (1963).

18. G. A. Hamilton, J. P. Friedman and P. M. Campbell, ibid.. 88, 5266 (1966).

19. G. A. Hamilton, J. W. Hanifin and J. P. Friedman, ibid. . 5269.

20. G. A. Hamilton, Advan. Enzymol., 32, 55 (1969).

21. S. Udenfriend, C. T. Clark, J. Axelrod and B. B. Brodie, J. Biol. Chem., 208, 731 (1954).

22. H. S. Mason, Advan. Enzymol., 19, 128 (1957).

23. F. Dewhurst and C. Calcutt, Nature, 191, 808 (1961).

24. G. A. Hamilton, R. L. Workman and L. Woo, J. Amer. Chem. Soc. . 86, 3390 (1964).

25. G. A. Hamilton, ibid., 3391.

26. C. Walling and s. Kato, I. Amer. Chem. Soc., 93, 4275 (1972).

27. R. Breslow and L. N. Lukens, J. Biol. Chem., 235, 292 (1960).

28. M. C. Flowers and H. M. Frey, I. Chem. Soc., 1961, 3547 .

29. R. Goupil and G. Mangency, Chim. Anal., 4I, 18 (1959).

30. N. D. Cheronis and T. S. Ma, "Organic Functional Group Analysis by Micro and Semi Micro Methods". Interscience Publishers, New York, 1964, p. 445.

31. D. N. Kramer and L. U. Tolentino, Anal. Chem., 43, 834 (1971).

32. H. O. Friestad, D. E. Ott and F. A. Gunther, Anal. Chem., 41, 1750 (1969).

33. S. Siggia, editor, "Instrumental Methods of Organic Functional Group Analysis". Wiley-Interscience, New York, 1972, p. 21. 
34. E. Emerson, J. org. Chem. , 8,417 (1943).

35. M. B. Ettinger, C. C. Ruchhoft and R. J. Lishka, Anal. Chem., 23, 1783 (1951).

36. R. J. Lacoste, S. H. Venable and J. C. Stone, Anal. Chem.. 31. 1246 (1959).

37. D. Svobodova, J. Gasparic and I. Novakova, Czech. Coll. Chem. Commun. 35, 31 (1970).

38. D. Svobodova and J. Gasparic, ibid., 1567.

39. D. Svobodova and J. Gasparic, Mikrochim. Acta, 1971. 384 .

40. H. D. Gibbs, J. Biol. Chem. , 72, 649 (1927).

41. M. B. Ettinger and C. G. Ruchhoft, Anal. Chem., 20, 1191 (1948).

42. G. Gorbach, O. G. Koch and G. Dedic, Mikrochim. Acta, 1955, 882 .

43. G. G. Guilbault, D. N. Kramer and E. Hackley, Anal. Chem.. 38, 1897 (1966).

44. J. C. Dacre, Anal. Chem., 43, 589 (1971).

45. E. F. Mohler, Jr. and L. N. Jacob, Anal. Chem., 29, 1369 (1957).

46. S. Siggia and J. E. Smola, Anal. Chem., 44, 532 (1972).

47. M. Hanriot, Compt. Rend., 102, 1250 (1886).

48. A. Jonescu, J. Pharm. Chim., 29, 533 (1909).

49. J. R. Nicholls, Analyst, 53,19 (1928).

50. F. W. Edwards, H. R. Nanji and M. K. Hassan, Analyst, 62, 172 (1937).

51. N. L. Allport and J. E. Brocksopp, "Colorimetric Analysis", Vol. II, 2nd ed., Chapman and Hall, London, 1963, p. 52.

52. J. Bartos, Ann. Pharm. Fr., 27, 759 (1969).

53. J. Bartos, Chimie Analytique, 53, 384 (1971).

54. H. D. Dakin, Org. Syn., I, 143 (193.2). 
55. A. W. Ingersoll, ibid., 304.

56. J. B. Cohen and H. W. Dudley, J. Chem. Soc., 97, 1732 (1910).

57. S. Kanno, J. Pharm. Soc. Japan, 72 , II93 (1954).

58. N. H. Cromwell, R. D. Babson and C. E. Harris, I. Amer. Chem. Soc., 65, 312 (1943).

59. F. W. Hoffman and H. D. Weiss, ibid., 79, 4759 (1956).

60. F. L. Cline and E. E. Reid, ibid., 49, 3153 (1927).

61. H. Adkins and R. Billica, I. Amer. Chem. Soc., 70, 695 (1948).

62. L. C. King, M. McWhirter and D. M. Barton, J. Amer. Chem. Soc., 67, 2089 (1945).

63. E. H. Woodruff, J. Amer. Chem. Soc., 66, 1799 (1944).

64. I. A. Pearl, J. org. Chem., 12, 85 (1947).

65. R. R. Dreisbach and S. A. Shrader, Ind. Eng. Chem., 4l, 2879 (1949).

66. w. w. Hartman, org. Syn.. I, 169 (1932).

67. C. Graebe, Ber., 35,2747 (1902).

68. S. E. Hunt, J. I. Jones, A. S. Lindsey, D. C. Killoh and H. S. Turner, J. Chem. Soc.. 1958, 3152.

69. Badische Anilin and Soda-Fabrik (L. Weschky, inventor), Ger. 805,644, May 25, 1951; Chem. Abstr. . 45, 9227e.

70. A. Klemenc, Monatsh., 35, 85 (1914).

71. J. B. Fishman, J. Amer. Chem. Soc. , 42, 2288 (1920).

72. R. H. F. Manske, Org. Syn., I, 369 (1932).

73. W. Robertson, I. Chem. Soc.. 81,1477 (1902).

74. J. C. Duff, I. Chem. Soc., 1941, 547.

75. L. F. Fieser and H. T. Thompson, I. Amer. Chem. Soc. 61, 376 (1939).

76. L. C. King, M. McWhirter and D. Barton, J. Amer. Chem. Soc. . 67, 2088 (1945). 

77. R. B. Woodward and W. E. Doering, J. Amer. Chem. Soc.
67,860 (1945).

78. J. Controulis, M. C. Rebstock and H. M. Crooks, Jr., I. Amer. Chem. Soc., 71, 2463 (1948).

79. R. Adams and F. L. Cohen, org. Syn.. I, 235 (1932).

80. P. K. Smith, "Acetophenetidin", Interscience Publishers, New York, 1958, p. 4.

81. F. K. Thayer, org. Syn. , 1, 12 (1932).

82. A. L. Klebanskii and A. L. Lemke, J. Applied Chem. .8 ,

83. L. H. Welsh, I. Amer. Chem. Soc., 69, 128 (1947).

84. R. Derrot and A. Barghon, Bull. Soc. Chim. France,
1951, 278.

85. A. Watanabe and H. Kamio, J. Pharm. Soc. Japan, 62,

86. E. I. Rosenblum and w. S. Taylor, J. Pharm. Pharmacol.,
․, 656 (1954).

87. R. T. Brittain, P. F. D'Arcy and J. H. Hunt, Nature,
183, 734 (1959).

88. A. I. Vogel, "Practical Organic Chemistry", 3rd ed., London, 1959, p. 577.

89. J. T. Carswell, J. Amer. Pharm. Assoc.. 16, 306 (1927).

90. L. F. Fieser and M. Fieser, "Reagents for organic Synthesis", John Wiley and Sons, Inc.., New York, 1967, p. 712 .

91. J. A. Huerta and R. Gonzalvo, Mon. Farm. y. terap. (Madrid), 58, 6 (1952); Chem. Abstr. 느, 4729 d.

92. A. Kofler, Mikrochim. Acta, 33, 4 (1947).

93. M. R. Natarajan and S. Swaminathan, I. Amer. Chem.
Soc. 69,2560 (1947).

94. R. G. Bates, J. Res. Nat. Bur. Stand., 66A, 179 (1962).

95. J. March,"Advanced Organic Chemistry: Reactions, Mechanisms' and Structures", McGraw-Hill, New York, 1968, p. 241 . 
96. J. H. Wang, J. Amer. Chem. Soc., 77, 4715 (1955).

97. D. French, Advan. Carbohyd. Chem., 12, 189 (1957).

98. J. A. Thoma and L. Stewart, "Starch: Chemistry and Technology", Vol. I, R. L. Whistler and E. F. Parschall, Ed., Academic Press, New York, 1965, p. 209.

99. A. Hybl, R. E. Rundle and D. E. Williams, I. Amer. Chem. Soc. , 87, 2779 (1965).

100. F. Cramer, W. Saenger and H. Ch. Spatz, ibid., 14 (1967).

101. M. L. Bender, R. L. Van Etten, G. A. Clowes and

J. F. Sebastian, ibid., 88, 2318 (1966).

102. N. Hennrich and F. Cramer, ibid., 87, 1121 (1965).

103. J. L. Lach and T. F. Chin, J. Pharm. Sci., 53, 924 (1964).

104. R. L. Van Etten, J. F. Sebastian, G. A. Clowes and M. L. Bender, I. Amer. Chem. Soc., 89, 3242 (1967).

105. R. L. Van Etten, G. A. Clowes, J. F. Sebastian and M. L. Bender, ibid., 3253.

106. R. Breslow and P. Campbell, ibid., 91, 3085 (1969).

107. R. Breslow and P. Campbell, Bioorg. Chem., I, 140 (1971).

108. F. Cramer, Angew. Chem. Internat. Edit., 5,601 (1966).

109. R. Breslow and L. E. Overman, I. Amer. Chem. Soc., 92, 1075 (1970). 


\section{7 -}

VII. APPENDICES 
APPENDIX A

STUDIES ON THE ANAIYSIS OF BARBITURIC ACID DERIVATIVES VIA OXIDATION BY DRUG-METABOLIIZING ENZYMES 
Studies on the Analysis of Barbituric Acid Derivatives via Oxidation by Drug-Metabolizing Enzymes

The potential utility of enzymes as analytical reagents has not yet been extensively realized in pharmaceutical. analysis, although they possess the desirable properties of specificity and sensitivity. The idea that these properties might be exploited for the analysis of drugs by using the very enzymes responsible for metabolizing them, directed attention to the oxidases of the microsomal-liver fraction. The choice of barbituric acid derivatives as enzyme substrates was largely based on a knowledge of their metabolic pathways. The metabolism of barbiturates has been reviewed by Mark (1) and Raventos (2). The oxidation of drugs by liver microsomes has been the subject of extensive investigations; key references are provided in "Microsomes and Drug oxidations", edited by Gillette, et al. (3).

The side-chain oxidation of barbiturates seems to be the main metabolic route $(1,2)$ and it therefore should provide the best possibility for obtaining an active enzyme from liver microsomes. Cooper and Brodie (4) observed that hexobarbital (5-cyclohexenyl-3,5-dimethyl barbituric acid) was oxidized to keto-hexobarbital (5-(3-oxo-1-cyclohexenyl)3,5-dimethyl barbituric acid) by a rabbit liver microsomal fraction. Both $\mathrm{O}_{2}$ and NADPH (TPNH, reduced triphosphopyridine nucleotide) were required. Further investigation by Tsukamoto $(5,6)$ revealed that the metabolic route leading to the ketone 
occurred by two successive reactions, given by Eqs. (A-I) and $(A-2)$, in which the first step was an NADPH-dependent liver microsomal hydroxylation of the cyclohexenyl group (Eq. A-1), and the second (Eq. A-2) was further oxidation to its ketone derivative by the soluble liver fraction. ${ }^{1}$ Both the cofactors NAD and NADP were found to possess the same activity<smiles>CN1C(=O)NC(=O)C(C)(C2=CCCCC2)C1=O</smiles><smiles>CC(C)NOc1ccccc1</smiles><smiles>Cn1c(=O)[nH]c(=O)c(=O)c(=O)n1C</smiles><smiles>CC1CCC(O)CC1=O</smiles>

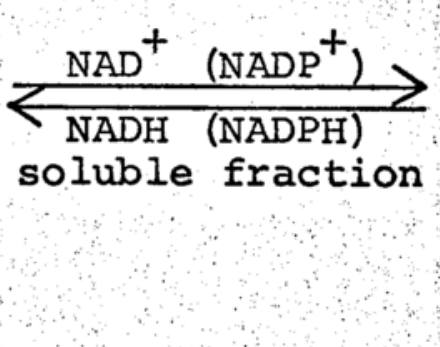<smiles>CN1C(=O)C2CCC(C)(C(=O)NC2=O)C1=O</smiles>

for the interconversion between keto-hexobarbital and its hydroxy precursor. This enzyme fraction also catalyzed the reduction of keto-hexobarbital to the hydroxy derivative in the presence of the reduced pyridine nucleotides (6). Purification of the $\mathrm{NAD}^{+}$-dependent dehydrogenase in the soluble rabbit liver fraction established its specificity for the cyclohexen-3-ol group (7).

I The microsomal fraction and soluble fraction of the rabbit liver are separable by centrifuging at 105,000 $\mathrm{x} g$ for $\mathrm{I}$ hr. The precipitate constitutes the microsomal fraction. 
Both $\omega$ - and $(\omega-1)$-oxidations of pentobarbital and secobarbital are further examples of side-chain oxidations of barbiturates that have been studied in a cell-free rabbit liver homogenate ${ }^{2}(8-10)$. A two-step process was again observed, the first involving an NADPH-mediated microsomal hydroxylation to form an alcohol, followed by further oxidation in the soluble liver fraction when NAD (or NADP) was present.

These literature results suggested that if an enzyme preparation from liver microsomes could be prepared (in as purified form as possible) that is capable of carrying out reactions on barbituric acid derivatives, then it might be possible to design sensitive and specific assays for the barbiturates based upon these metabolically important reactions.

The following procedure was used to obtain a rabbit liver preparation: Male, albino rabbits, about $4 \mathrm{lb}$ in weight, were stunned by a blow on the head, exsanguinated, and the livers removed and homogenized in 2 volumes of $0.1 \underline{\mathrm{M}}$ phosphate buffer, $\mathrm{pH} 7.4$, containing $30 \mathrm{mM}$ nicotinamide and $4 \mathrm{mM} \mathrm{MgCl}_{2}$, using a Potter-Elvehjem homogenizer. ${ }^{3}$ After centrifuging the homogenate at $600 \times \mathrm{g}$ (in a refrigerated centrifuge) for $10 \mathrm{~min}$ to remove nuclei and cells, the

2 Centrifugation of a liver homogenate at $9,000 \times \mathrm{g}$ for 20 min removes cells, nuclei, and mitochondria.

3 All manipulations after the liver was removed were done in a cold room at about $4^{\circ} \mathrm{C}$. 
supernatant was centrifuged at $9,000 \times \mathrm{g}$ for $20 \mathrm{~min}$ to remove mitochondria. The resulting supernatant was centrifuged at $105,400 \times \mathrm{g}$ for $\mathrm{I} \mathrm{hr}$ to obtain the soluble liver fraction and precipated microsomes. The microsomes were washed with $0.1 \mathrm{M}$ phosphate buffer and resuspended in 0.1 M phosphate buffer to a volume equivalent to that of the original liver. Reactions were followed by gas chromatographically determining the amount of unreacted barbiturate. 4 Both pentobarbital and hexobarbital were used as substrates. Incubations were carried out at $37^{\circ}$ with constant shaking. 5 Typical reaction conditions are presented in Table $\mathrm{A}-\mathrm{I}$.

Some results comparing the extent of metabolism of hexobarbital and pentobarbital in a 9,000 x g liver homogenate and a microsomal fraction are given below:

\begin{tabular}{lll} 
& \multicolumn{2}{c}{$\%$ Metabolism } \\
\cline { 2 - 3 } Substrate & $\frac{9,000 \mathrm{~g}}{20}$ & $\frac{\text { Microsome }}{15}$ \\
Hexobarbital & 20 & 15 \\
Pentobarbital & 16 & 10
\end{tabular}

4 The unreacted substrate was extracted with ethyl acetate from an acidified portion of reaction mixture, and the volume of ethyl acetate reduced to $2.0 \mathrm{ml}$. A $2 \mu \mathrm{l}$ aliquot was injected onto a $6^{\prime} \times 4 \mathrm{~mm}$ column containing $2 \%$ Hi-Eff $8 \mathrm{BP}$ on Gas Chrom $Q$ at a temperature of $250^{\circ}$. A BarberColeman model 5000 gas chromatograph was used.

5 A metabolite shaker (Metabolyte Water Bath Shaker, New Brunswick Scientific Co.) was used. 
Table A-I

Incubation Conditions for the Metabolism of Barbiturates. ${ }^{a}$

\begin{tabular}{ll}
\hline \multicolumn{1}{c}{ Compound } & Amount \\
\hline Glucose-6-Phosphate & 15 umoles \\
Glucose-6-Phosphate Dehydrogenase & 100 units \\
NADPH & $0.8 \mu$ moles \\
MgCl$_{2}$ & $50 \mu$ moles \\
Nicotinamide $^{\mathrm{b}}$ & $100 \mu$ moles \\
Enzyme Preparation & 4 cc \\
Substrate & $6-8 \mu$ moles \\
\hline
\end{tabular}

a $37^{\circ}$, one hr with shaking.

b Turns over NADPH.

c Microsomes or soluble fraction. 
Although the $9,000 \times \mathrm{g}$ fraction gave conversions comparable to literature values (4), it appears that the microsomal fraction was partially denatured during its preparation. This was a constant problem, and in fact, a microsomal fraction devoid of activity was common, even though the literature indicates that microsomal pellets could be stored at $-15^{\circ}$ for 2 months with no loss in activity (11).

The possibility of following the time course for reaction by monitoring the oxidation of NADPH at $340 \mathrm{~nm}$ was investigated. However, the turbidity imparted by a microsomal suspension created serious instrumental problems.

A major limitation with the use of a microsomal fraction, aside from its instability; is that the product of barbiturate metabolism, an alcohol, is difficult to detect, particularly in such small amounts (due, in part, to low yields). Although the $9,000 \times \mathrm{g}$ liver homogenate has the potential to produce an $\alpha, \beta$-unsaturated ketone (a structural feature that may provide a way to follow the reaction) its production is the result of a two-step process and low yields might be anticipated. It would appear then, based upon the above discussion, that the approach has little to recommend it, as a superior analytical method. The possibility exists, however, that the production of a reasonably pure, active microsomal preparation would overcome the limitations of instability of the enzyme preparation and of the low yields resulting from their catalysis. 


\section{References}

1. L. C. Mark, Clin. Pharmacol. Ther., 4, 504 (1963).

2. J. Raventos, J. Pharm. Pharmacol.. 217, 512 (1954).

3. J. R. Gillette, A. H. Conney, G. J. Cosmides, R. W. Estabrook, J. R. Fouts and G. J. Mannering, editors, "Microsomes and Drug Oxidations", Academic Press, New York, 1969.

4. J. R. Cooper and B. B. Brodie, J. Pharmacol. Exptl. Therap., 114, 409 (1955).

5. S. Toki, K. Toki and H. Tsukamoto, Chem. Pharm. Bull.. 10, 708 (1962).

6. K. Toki, S. Toki and H. Tsukamoto, I. Biochem., $\underline{53}, 43$ (1963).

7. K. Toki and H. Tsukamoto, ibid., 55, 142 (1964).

8. J. R. Cooper and B. B. Brodie, J. Pharmacol. Exptl. Therap. .120, 75 (1957).

9. K. M. Ikeda, M. Jacobson and A. H. Conney, ibid., 157, 220 (1967).

10. H. Ide, H. Yoshimura and H. Tsukamoto, Chem. Pharm. Bull.. 15, 411 (1967).

11. W. Levin, A. Alvares, M. Jacobson and R. Kuntzman, Biochem. Pharmacol.. 18, 883 (1969). 


\begin{abstract}
APPENDIX B
DETERMINATION OF HYDROXY COMPOUNDS BY DIMETHYLAMINOPYRIDINE-CATALYZED ACETYLATION
\end{abstract}


Determination of Hydroxy Compounds by Dimethylaminopyridine-Catalyzed Acetylation ${ }^{1}$

Pyridine-catalyzed acetylation (1) and phthalation (2) are standard methods for the determination of hydroxy compounds and other acylable substances. The mechanism involves nucleophilic catalysis with the intermediate formation of the acylpyridinium ion ( 3$)$, as shown in Equations $(B-1)$ and $(B-2)$ for the reaction of an alcohol with acetic anhydride.

$$
\begin{aligned}
& \mathrm{Ac}_{2} \mathrm{O}+\mathrm{N}^{\mathrm{W}} \underset{\mathrm{AcN}}{\mathrm{I}}+\mathrm{W}+\mathrm{OAC}^{-}
\end{aligned}
$$

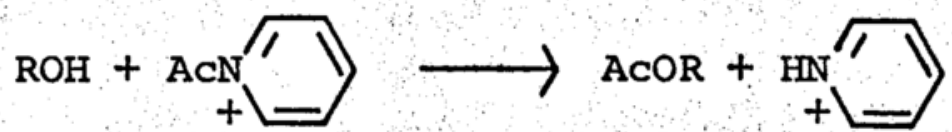

Although pyridine is an effective catalyst in such acylations, typical reaction times are 0.5 to $1 \mathrm{hr}$ at reflux temperature. Schenk, et al. (4) compared several tertiary amines as catalysts in the acetylation of cyclohexanol; they found that triethylenediamine is about 50 times more effective than pyridine. Because of the greater base strength of this saturated amine, it was necessary to modify the classical titrimetric finish (4). Triethylenediamine is also a better catalyst than is pyridine in the acylation of alcohols with the acid chloride of the 2,4-dinitrophenylhydrazone of

1 This appendix represents a paper by Kenneth A. Connors and Kenneth S. Albert (10). 
pyruvịc acid (5). Imidazole catalyzes acylations by pyromellitic dianhydride (6). Analytical acylations have been thoroughly reviewed by Mehlenbacher (7) and Schenk (8).

Recently Steglich and Höfle (9) have reported that 4dimethylaminopyridine is superior to pyridine as a catalyst for some synthetic acylations. The present note describes the application of 4-dimethylaminopyridine (DMAP) to the determination of hydroxy compounds by reaction with acetic anhydride.

\section{EXPERIMENTAL}

Materials. 4-Dimethylaminopyridine (Aldrich Chemical Company) was used directly.

Reagents. Acetic Anhydride Solution. $10 \mathrm{ml}$ of reagent grade acetic anhydride was mixed with $40 \mathrm{ml}$ of reagent grade pyridine. This reagent should be prepared fresh daily. DMAP Solution. $2 \mathrm{~g}$ of 4-dimethylaminopyridine was dissolved in enough pyridine to make $100 \mathrm{ml}$. Each $5-\mathrm{ml}$ aliquot of this solution contains $100 \mathrm{mg}$ of DMAP.

Procedure. A 2-3 milliequivalent sample of a hydroxy compound contained in $1.0 \mathrm{ml}$ of pyridine solution was pipetted into a $50-\mathrm{ml}$ erlenmeyer flask. $5.0 \mathrm{ml}$ of DMAP Solution followed by $2.0 \mathrm{ml}$ of Acetic Anhydride Solution were added, and the solution was well mixed. The stoppered flask was maintained at the selected temperature for an appropriate time 
(see Table B-I and discussion). $25 \mathrm{ml}$ of water was added to the flask and the contents were brought to room temperature. If the mixture was homogeneous, $5 \mathrm{ml}$ of $\underline{\text { n-butyl alcohol was }}$ added. Three drops of $1 \%$ phenolphthalein solution were added, and the solution was titrated to the pink color with standard $0.5 \underline{N}$ sodium hydroxide. A blank determination, omitting only the sample, was carried out in exactly the same way.

The milliequivalents of hydroxy compound contained in the sample taken for assay is given by $\underline{N}\left(v_{b}-v_{s}\right)$, where $v_{b}$ and $v_{s}$ are the volumes (in $\mathrm{ml}$ ) of sodium hydroxide solution of normality $\underline{N}$ required to titrate the blank and sample, respectively.

\section{RESULTS AND DISCUSSION}

Figure B-I shows the time course for acetylation of isopropyl alcohol in a pyridine-acetic anhydride mixture at different concentrations of DMAP. A profound catalysis of this esterification by DMAP clearly occurs. In the development of the analytical procedure pyridine has been retained as the solvent, though it makes little contribution to the overall catalysis.

An estimate of the relative catalytic efficiencies of DMAP and pyridine was obtained as follows. It is anticipated that the rate equation for acylation in a DMAP-pyridine-acetic anhydride mixture will be given by Equation $(B-3)$. 
$v=\left(k_{\mathrm{PYR}}[\mathrm{PYR}]+\mathrm{k}_{\mathrm{DMAP}}[\mathrm{DMAP}]\right)[\mathrm{ROH}]\left[\mathrm{Ac}_{2} \mathrm{O}\right]$

$v=\left(k_{P Y R}^{\prime}+k_{\text {DMAP }}^{\prime}\right)[R O H]\left[\mathrm{Ac}_{2} \mathrm{O}\right]$

$$
v=k_{o b s}[\mathrm{ROH}]\left[\mathrm{Ac}_{2} \mathrm{O}\right]
$$

From the titrimetric data the apparent second-order rate constant $k_{\text {obs }}$ could be obtained. The plots showed some deviations from strict second-order kinetics, but for the present purpose it was possible to obtain these estimates: for reaction with iso-propyl alcohol at $54^{\circ} \mathrm{C}, \mathrm{k}_{\mathrm{PYR}}=3.6 \times 10^{-6} \underline{\mathrm{M}}^{-2}$ $\sec ^{-1}$ and $k_{\text {DMAP }}=7 \times 10^{-2} \underline{M}^{-2}-\mathrm{sec}^{-1}$. DMAP therefore is about $2 \times 10^{4}$ times more effective than is pyridine as a catalyst in this reaction. This rate enhancement may be a consequence of stabilization of the acylpyridinium ion, or rather of the transition state leading to it, as shown below.

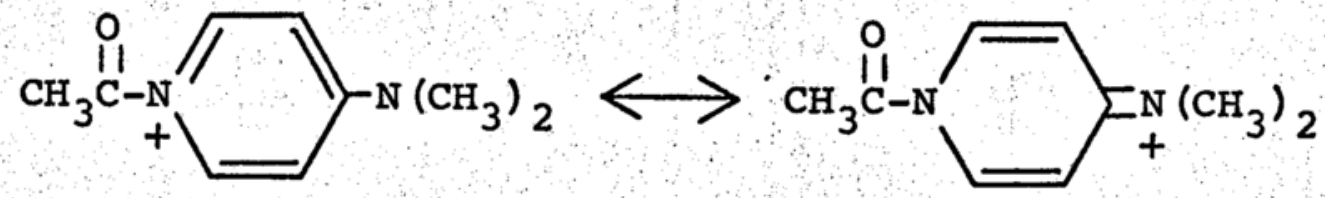

Table B-I lists analytical results for the DMAP-catalyzed acetylation of various hydroxy compounds. The accuracy and precision of the method are comparable with those of other acylation procedures. Given a sample compound, the analyst has available for manipulation three experimental variables, namely, DMAP concentration, temperature, and reaction time. Sufficient variation in these quantities is represented in Table B-I for the analyst to select appropriate conditions to 
Table B-I

Analytical Results of DMAP-Catalyzed Acetylation of Hydroxy Compounds

\begin{tabular}{|c|c|c|c|c|c|}
\hline \multirow[b]{2}{*}{ Sample compound } & \multirow{2}{*}{$\begin{array}{l}\text { Amount } \\
\text { DMAP } \\
\text { (mg) }\end{array}$} & \multirow{2}{*}{$\begin{array}{l}\text { Temp. } \\
\left({ }^{\circ} \mathrm{C}\right)\end{array}$} & \multirow{2}{*}{$\begin{array}{l}\text { Reaction } \\
\text { time } \\
\text { (min) }\end{array}$} & \multicolumn{2}{|c|}{ Percent recovery } \\
\hline & & & & Mean $^{\mathrm{b}}$ & std. dev. \\
\hline n-Propyl alcohol & 50 & 54 & 20 & 100.1 & 0.2 \\
\hline iso-Propyl alcohol & 50 & 54 & 20 & 100.1 & 0.4 \\
\hline Benzyl alcohol & 50 & 54 & 20 & 100.4 & 0.6 \\
\hline n-Butyl alcohol & 100 & 54 & 10 & 99.0 & 0.5 \\
\hline Phenol & 100 & 54 & 10 & 101.2 & 0.3 \\
\hline Cinnamyl alcohol & 100 & 54 & 10 & 98.1 & 0.4 \\
\hline iso-Butyl alcohol & 100 & 70 & 5 & 100.6 & 0.4 \\
\hline Ethylene glycol ${ }^{\mathrm{C}}$ & 100 & 70 & 5 & 99.2 & 0.2 \\
\hline p-Methoxyphenol ${ }^{d}$ & 100 & 70 & 10 & 99.4 & -- \\
\hline
\end{tabular}
a Amount of DMAP in the $8-\mathrm{ml}$ reaction volume.
b Mean of 3 determinations except as noted.
c Both hydroxy groups acetylated.
d Two determinations. 
suit his requirements. The attempted determination of tertbutyl alcohol ( $100 \mathrm{mg}$ DMAP, $70^{\circ}$ ) gave $26 \%$ acetylation in 75 min reaction time.

A yellow color in the presence of high DMAP concentrations limits the accuracy of the visual endpoint detection, and it appears that in the procedure as described $100 \mathrm{mg}$ of DMAP in the 8-ml assay volume is about the maximum practicable amount. Higher concentrations of DMAP give increasingly erratic results, which are associated with definite consumption of reagent even in the blank solution. Possibly this very powerful catalyst is promoting c-acylation of acetic anhydride in a Perkin-type reaction.

The DMAP-catalyzed method given here appears to be the most reactive of available anhydride acylation methods utilizing nucleophilic catalysis. Extension of DMAP catalysis to other acylating agents, types of samples, and finish methods should be analytically fruitful. 


\section{References}

1. C. L. Ogg, W. L. Porter, and C. 0. Willits, Ind. Eng. Chem.. Anal. Ed., 17, 394 (1945).

2. P. J. Elving and B. Warshowsky, ibid., 19, 1006 (1947).

3. A. R. Fersht and W. P. Jencks, J. Amer. Chem. Soc. 91. 2125 (1969).

4. G. H. Schenk, P. Wines, and C. Mojzis, Anal. Chem., 36, 914 (1964).

5. D. P. Schwartz, Anal. Biochem., 38, 148 (1970).

6. B. H. M. Kingston, J. J. Garey, and W. B. Hellwig, Anal. Chem., 41, 86 (1969).

7. V. C. Mehlenbacher, Org. Anal. I I, I (1953).

8. G. H. Schenk, Organic Functional Group Analysis, Pergamon Press, Oxford, 1968, Chapters 3, 4.

9. W. Steglich and G. Höfle, Angew. Chem. Intern. Ed.. 8 , 981 (1969).

10. K. A. Connors and K. S. Albert, submitted to Anal. Chem. 


\section{Figure Legend}

Figure B-1. Time course for acylation of iso-propyl alcohol by acetic anhydride at $54^{\circ}$ and varying DMAP concentration. Solvent, $1: 4$ $\mathrm{Ac}_{2} \mathrm{O}$-pyridine (parts by volume), DMAP concentration (slowest to fastest reactions), zero, $4.65 \times 10^{-3} \mathrm{M}$ and $4.48 \times 10^{-2} \underline{\mathrm{M}}$. 

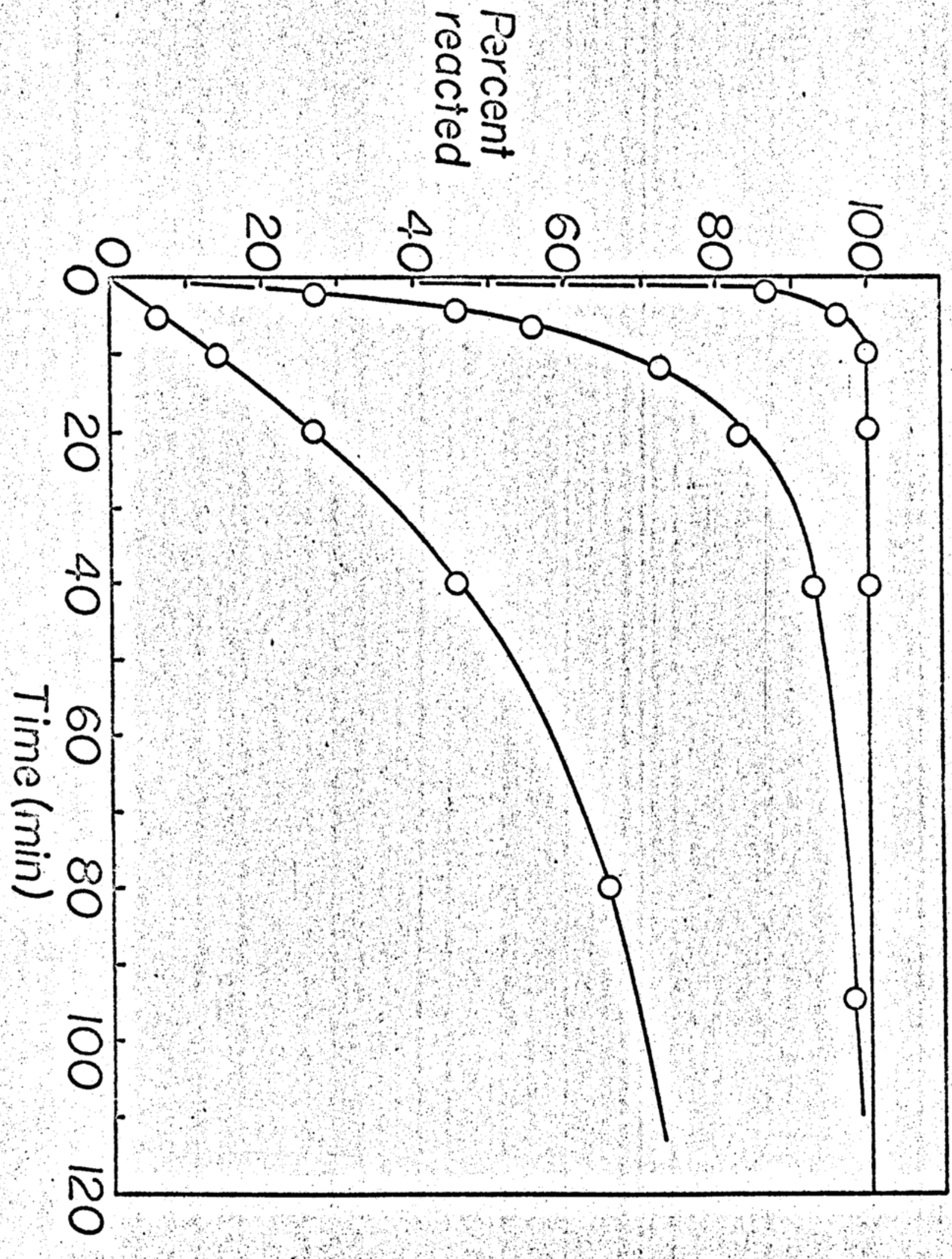


\section{KENNETH STEPHEN ALBERT}

\section{Personal Data}

Date of Birth: November 12, 1941

Marital Status: Married

Wife's Name: Evelyn

Children: Heidi Ellyn

Address: 2237 Stone Road, Ann Arbor, Michigan

\section{Education}

University of Texas, Austin, Texas, B.A. in Chemistry (1963).

University of Wisconsin. Madison, Wisconsin, M.S. in Pharmacy (1969).

University of Wisconsin, Madison, Wisconsin, Ph.D. in Pharmacy (1972); major in Pharmaceutics and Pharmaceutical Analysis, minor in Analytical Chemistry; thesis research under the direction of Kenneth A. Connors.

\section{Professional Experience}

Junior Scientist, Analytical Research Division, Ciba Pharmaceutical Company, Summit, New Jersey, 1963-1966.

Associate Scientist, Analytical Research Division, Bristol-Meyers Products Division, Hillside, New Jersey, Summer, 1967.

Instructor in Chemistry, Newark College of Engineering, Newark, New Jersey, 1967-1968.

Associate Scientist, Applied Analytical Division, Warner-Lambert Research Institute, Morris Plains, New Jersey, 1968.

Lecturer in Pharmacy, University of Wisconsin, Madison Wisconsin, Summer, 1971.

Postdoctoral research, Pharmacy Department, University of Michigan, Ann Arbor, Michigan, beginning August, 1972, with John G. Wagner. 
Associations

American Pharmaceutical Association

American Chemical Society

American Association for the Advancement of Science

Sigma Xi

Phi Lambda Upsilon

\section{Publications}

1. Separation and Determination of Chlorpheniramine and its Metabolites from Urine, $K$. Albert and J. J. Windheuser, J. Pharm. Sci., 57, 2085 (1968).

2. Aromatic Hydroxylation as an Analytical Reaction, K. A. Connors and K. S. Albert, Anal. Chem.. 44, 879 (1972).

3. Determination of Hydroxy Compounds by 4-Dimethy1aminopyridine-Catalyzed Acetylation, K. A. Connors and K. S. Albert, submitted to Anal. Chem.

4. Colorimetric Determination of Some Aromatic Drugs by Hydroxylation, K. S. Albert and K. A. Connors, in preparation.

5. Student Experiments in Pharmaceutical Analysis. VIII. Determination of Hydroxy Compounds by Acylation, K. S. Albert, K. A. Connors and H. Corrick, in preparation. 